\title{
10. SITES 143 AND 144
}

The Shipboard Scientific Party ${ }^{1}$

\begin{abstract}
Site 143 lies about halfway down the lower part of the Demerara Rise and Site 144 lies near the top just below the edge of the flat upper plateau, about $3 \mathrm{~km}$ southwest of Site 143 and $400 \mathrm{~km}$ north of the Guiana coast.

Only one core of Cretaceous material was recovered at Site 143 (at sea floor) before technical problems necessitated abandoning the site.

A sequence of Oligocene at sea floor to Early Albian to Late Aptian sediments at a depth of 325 meters was recovered at Site 144. Three small hiatuses were detected; one in the Late Eocene at 46 meters; one at the Tertiary Cretaceous boundary at 147 meters; and one between Late Campanian and Early Santonian at 180 meters.

The succession at Site 144 consists of Oligocene to Paleocene foraminifera-nannoplankton chalk ooze with Radiolaria in the Eocene. Paleocene and Maestrichtian sediments are mainly zeolitic marl. At 180 meters there is a pronounced lithology change to zeolitic calcareous carbonaceous shale, with a strong $\mathrm{H}_{2} \mathrm{~S}$ odor, which is mainly of Senonian to Turonian age. Marl, quartzose marlstone, shelly limestone, and carbonaceous clay were deposited from Cenomanian to Aptian times.

Sediments formed at the site of deposition, and those from neighboring marginal shelves and the continent, indicate regional subsidence over a long period of time. The area of Site 144 was a poorly oxygenated environment in the Cretaceous with abundant quartz, carbonate, and organic matter introduced from upslope into this area of clay deposition. In the Tertiary, fertility increased and pelagic constituents began to dominate in a well-oxygenated environment at the sea floor.
\end{abstract}

\section{SITE DATA}

\section{Hole 143A}

Time: 2200 November 19,1970

0130 November 21,1970

Position: $09^{\circ} 28.45^{\prime} \mathrm{N}$

$$
54^{\circ} 18.71^{\prime} \mathrm{W}
$$

Water Depth: 11,460 feet

$$
1,845 \text { nominal fathoms }
$$$$
3,493 \text { meters }
$$

Cores Taken: One core

\section{Hole 144}

Time: 1658 November 23,1970

1206 November 27,1970

Position: $09^{\circ} 27.23^{\prime} \mathrm{N}$

$$
54^{\circ} 20.52^{\prime} \mathrm{W}
$$

\footnotetext{
${ }^{1}$ D. E. Hayes, Lamont-Doherty Geological Observatory of Columbia University, Palisades, New York; A. C. Pimm, Scripps Institution of Oceanography, La Jolla, California; W. E. Benson, National Science Foundation, Washington, D. C.; W. H. Berger, Geologisch Palaeontologisches Institut-Kiel, Kiel, Germany; U. von Rad, Bundesanstalt für Bodenforschung-Hannover, Hannover, Germany; P. R. Supko, Scripps Institution of Oceanography, La Jolla, California; J. P. Beckmann, Geologisches Institut-Zurich, Zurich, Switzerland; P. H. Roth, Geologisches Institut-Zurich, Zurich, Switzerland.
}

Water Depth: 9,702 feet

$$
\begin{aligned}
& \text { 1,573 nominal fathoms } \\
& \text { 2,957 meters }
\end{aligned}
$$

Cores Taken: Seventeen cores

\section{SITES 143 AND 144 - BACKGROUND, SURVEY AND OPERATIONS}

Sites 143 and 144 are located respectively on the steep lower part and near the flat upper plateau of the Demerara Rise (Figure 1). The Demerara Rise is a prominent topographic terrace that extends northward as part of the continental slope and margin of the Guiana coastal area. One sample of Cretaceous and one of Jurassic marine sediments have been collected on the flank of the rise. One, a dredge sample from the Kane has been reported by Fox et al. (1970). A piston core (located close to Site 143) taken by geologists at Koninklijke/Shell and reported to the DSDP as privileged information, penetrated silty shales only 2 meters below the sea floor and was dated as Barremian to Aptian on the basis of sporomorphs.

In popular pre-drift continental reconstructions, the Guiana and Venezuela coasts of South America define one boundary of a proto-Atlantic ocean and according to these hypotheses should have extremely old marine sediments deposited there. A primary objective of Sites 143-144 was to drill and sample very old sediments. Seismic reflection records (Figures 2, 3,4), bathymetric data, and the bottom 


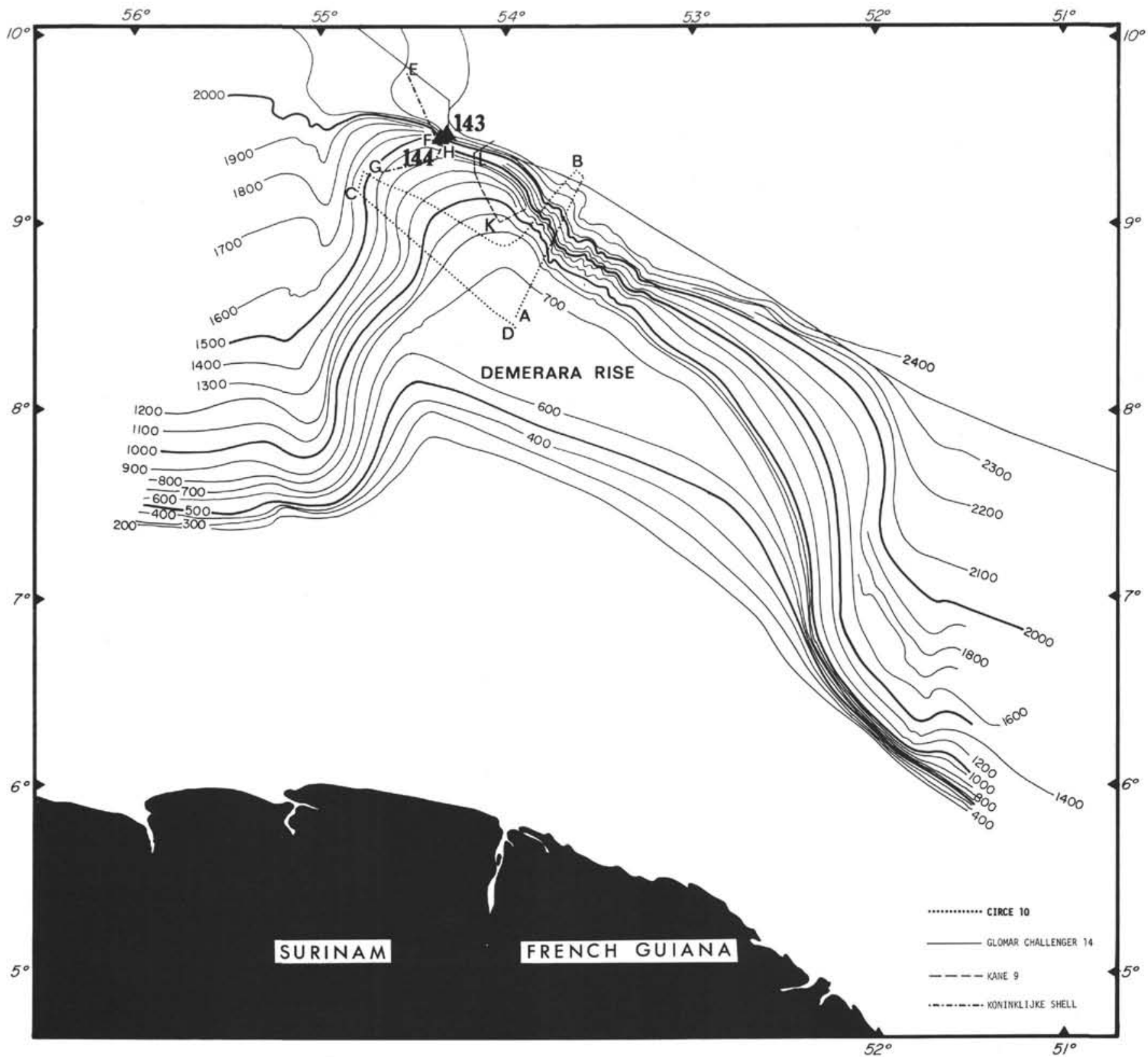

Figure 1. Location map for Sites 143-144. Contours in nominal fathoms, modified from a map supplied by Koninklijke Shell (pers. comm.). Letters key seismic profiles shown in Figures 2, 3 and 4.

samples all indicate that progressively older strata outcrop along increasing depths of the northeast flank of the Demerara Rise. Resistant layers are suggested by small topographic terraces (Figure 4) along the flank. Care was taken to locate the sites slightly above such terraces to help ensure the presence of sufficient soft material to spud into.

Privileged seismic data provided by Shell (Figure 2) indicate that a prominent angular unconformity exists at a depth of about 400 to 500 meters below the flat surface of the Demerara Plateau. The surface defining the unconformity is widespread and now lies at a depth of about 3000 meters below sea level. The considerable relief on the north flank and our knowledge of the precise water depth at the drill site allows us to accurately locate Site 144 in relation to the Shell seismic profile and the surface of the unconformity. A detailed interpretation of the Shell seismic profile is shown as part of the composite Figure 5 .

Seismic Reflection Data: Koninklijke/Shell Challenger Major reflector $\quad 0.32 \mathrm{sec} \quad$ None (top of unconformity) Reflector $0.80 \mathrm{sec}$

The Demerara Rise was approached from the southeast to a point about $5 \mathrm{~km}$ from proposed Site 143. The ship's course was then altered to $225^{\circ}$ to duplicate one of the SHELL geophysical lines. It was intended to make a continuous traverse from the flat basin floor onto the northern slope of the Demerara Rise, however, the best available topographic base map for this area was in error and the Challenger turned at the base of the slope instead of within the flat basin floor. 
B

A 0
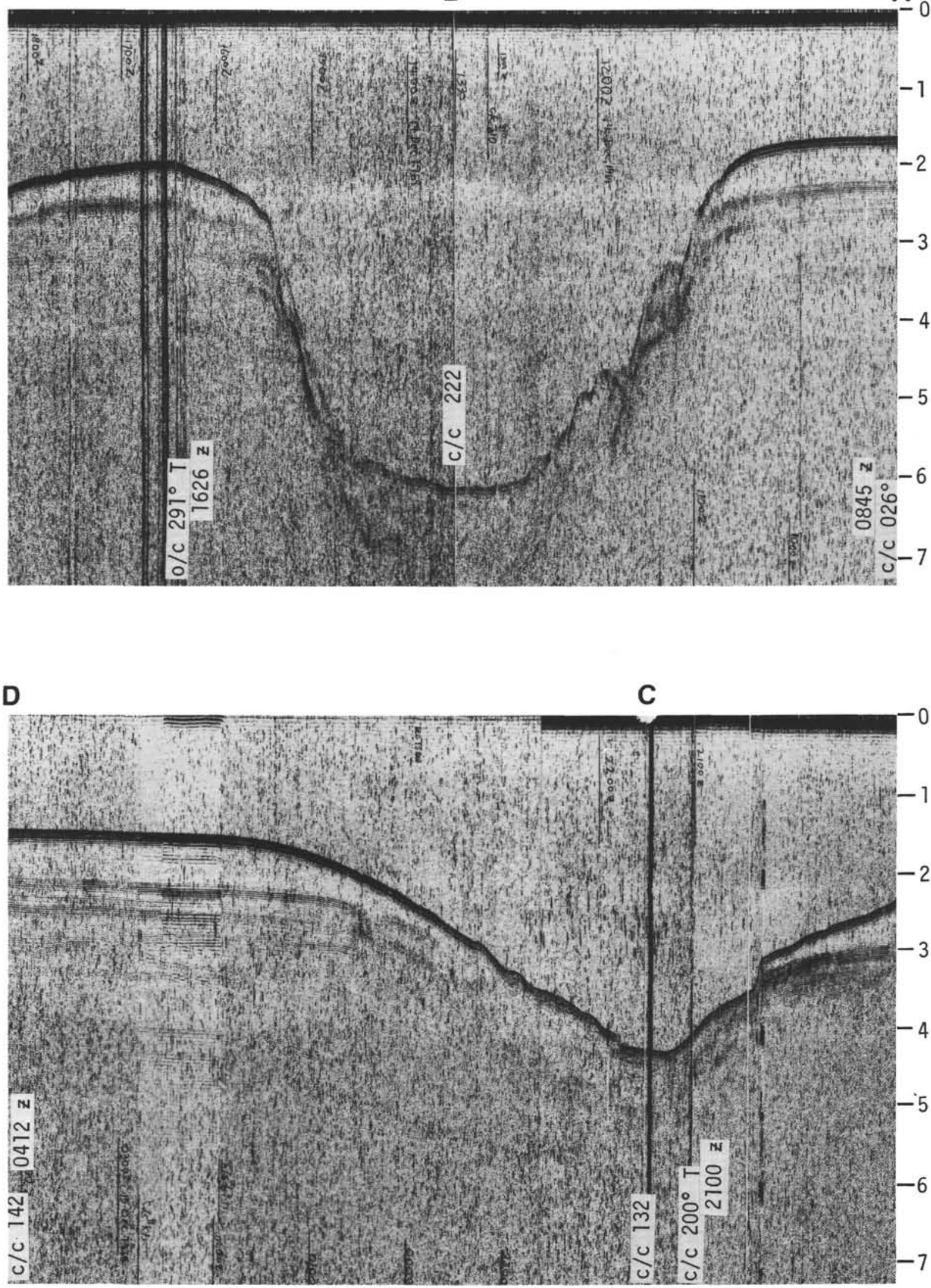

Figure 2. Seismic records from Circe 10. (J. Curray, pers. comm.) see Figure 1 for profile locations. Profiles illustrate the regional sediment disposition in the area of Sites 143-144. 

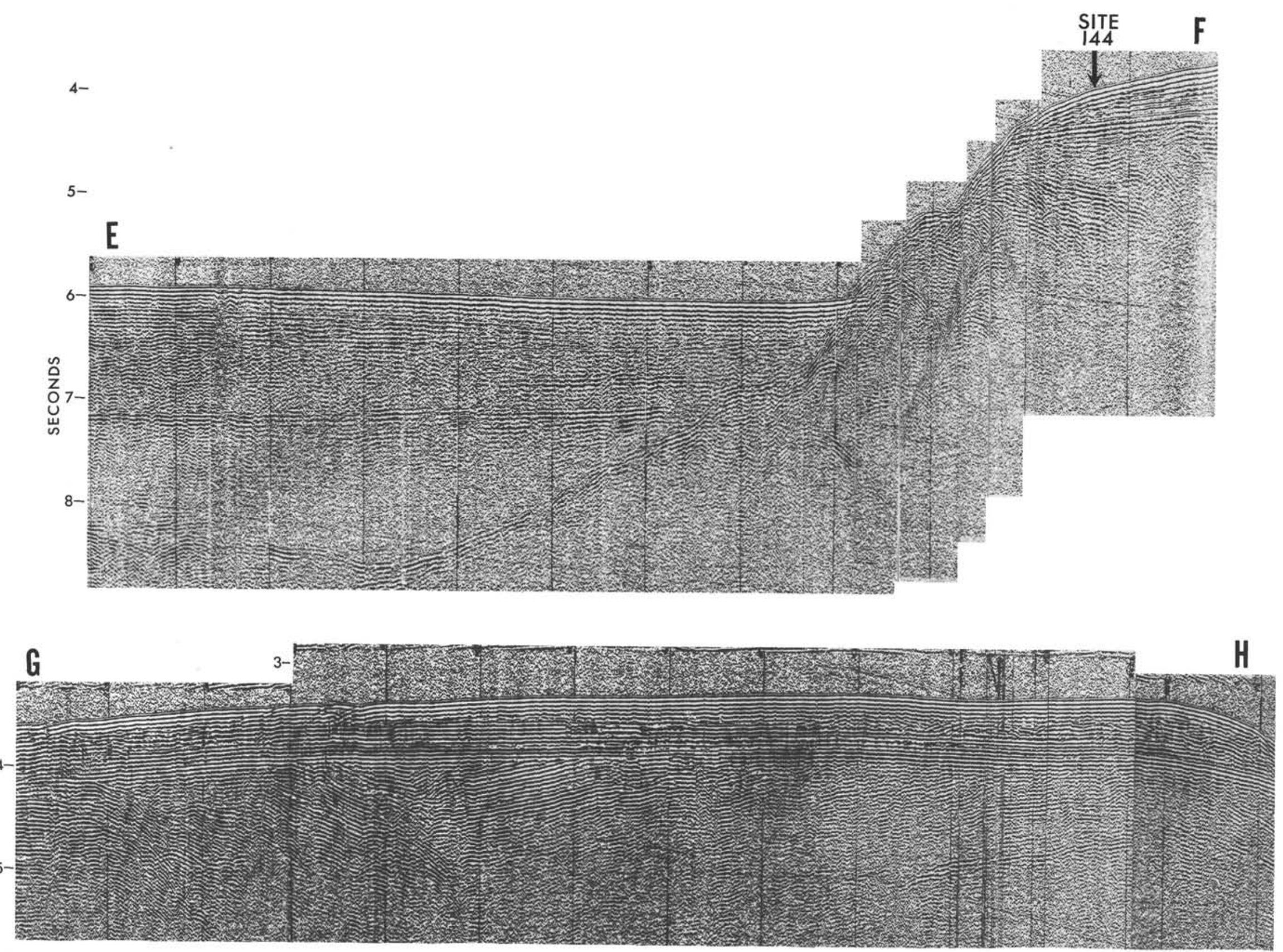

Figure 3. Seismic records from Koninklijke Shell (pers. comm.) see Figure 1 for profile location. Profile E-F passes almost directly over Sites 143 and 144. The estimated positions of these sites relative to the Shell profiles are indicated. Note the pronounced irregular unconformity shown on Profile GH at about 0.5-0.6 sec depth. 


\section{K}

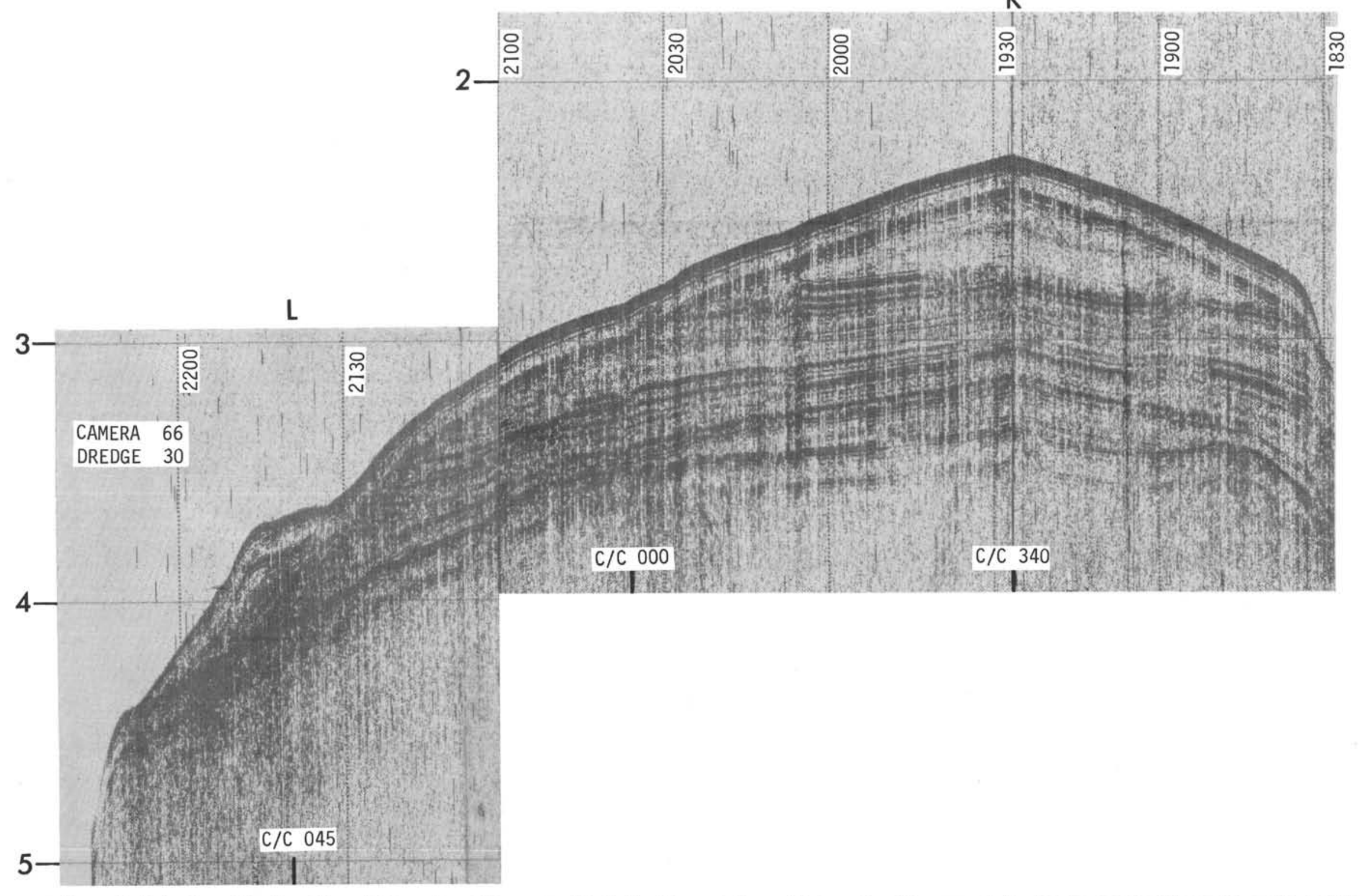

Figure 4. Seismic profiles from Kane 9. (Lowrie and Escowitz, 1969). See Figure 1 for profile location. The arrow indicates the comparable position of Site 144 relative to the Kane profile. Note the apparent outcropping of deep strata defined by prominent reflectors and expressed as topographic benches. 


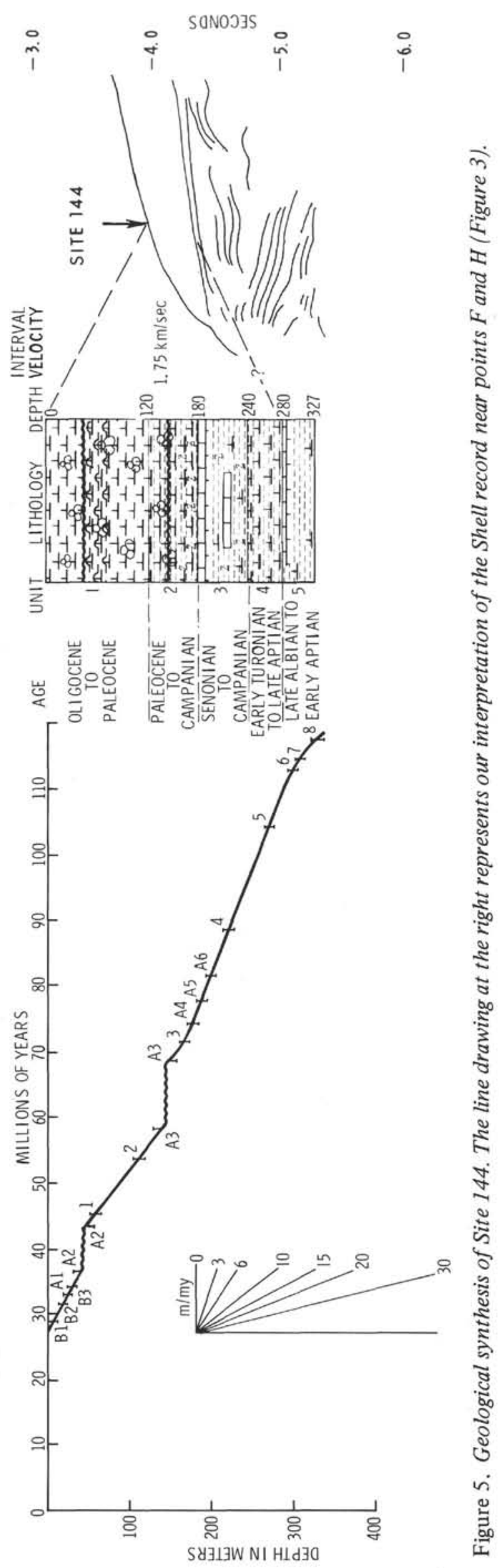

As the Challenger was unable to obtain a good quality seismic profile up the steep slope of the rise, the final selection of Site 143 had to be made on the basis of water depth and the bottom character as seen on the precision depth recorder. Two holes (143 and 143A) were attempted at the site selected and three more $(143 \mathrm{~B}, \mathrm{C}, \mathrm{D})$ at a location 300 meters downslope. These latter were made in an attempt to avoid moderately hard formations at or close to the surface, but the deepest penetration obtained was only 40 meters (Hole 143C). Only one core in Hole 143A was recovered from all five holes attempted at this site.

As Site 144 was only $4 \mathrm{~km}$ southwest of Site 143 , the geophysical gear was not towed and again site selection was made using the depth recorder. Operations proceeded smoothly at this site and a summary of drilling and coring is given in Figure 6 and Table 1.

\section{SITE 143}

\section{General}

Only one core was recovered at this Site. It is highly contaminated with Recent material, but also contains some Cretaceous foraminifera and nannoplankton. These indicate an age of Late Albian to Cenomanian. The fossil assemblage is very similar to that of Core 5 in Site 144. The sediment recovered was conglomeratic sand and gravel. The lithic fragments were as follows: green mudstone, subangular medium-grained quartz sandstone, $\mathrm{Fe}$ oxide crusts associated with greenish gray shale, $\mathrm{Fe} / \mathrm{Mn}$ fragments, and silty quartzose fine-grained sandstone. The disaggregated nature of the core material and its very heterogeneous nature strongly suggest a talus deposit. The age diagnostic fossils for this core is given in Table 2 .

\section{SITE 144 BIOSTRATIGRAPHY}

\section{General}

Abundant planktonic foraminifera, nannoplankton and Radiolaria occur in the chalks and marls in the upper part of the holes drilled at this site. Rich Oligocene microfaunas and nannofloras were recovered from 144B-1, B-2, A-2, B-3 and A-2 (upper part). Some admixed Quaternary, Pliocene, and Miocene planktonic foraminifera in 144B-1, and a trace of Upper Miocene with nannoplankton at the top of $144 \mathrm{~A}-2$, indicate that younger sediments are now, or were at one time, overlying the Oligocene in this area. An unconformable contact of Lower Oligocene or Middle Eocene was recovered in 144A-2. Below the Middle Eocene of 144-1, Upper Paleocene sediments with planktonic foraminifera nannoplankton and radiolarians were encountered in 144-2 and 144A-3 where they overlie Lower Maestrichtian. Rich Maestrichtian radiolarian assemblages occur in 144-3. Another unconformity was found between 144A-4 and A-5 where Upper Campanian rests on Coniacian to Santonian. Monotonous assemblages of small planktonic foraminifera and fairly rich nannoplankton occur in 144A-5, A-6 and 4. Mainly benthonic foraminifera, common ostracods, pelecypods, and gastropods (all indicating shallow water), and rather poor assemblages of nannoplankton were found in 144-6, 7 and 8. 
TABLE 1

Drilling and Coring Record for Site 144

\begin{tabular}{|c|c|c|c|}
\hline Description & $\begin{array}{c}\text { Interval Below } \\
\text { Sea Floor } \\
\text { (m) }\end{array}$ & $\begin{array}{l}\text { Core Recovery } \\
\text { (m) }\end{array}$ & $\underset{(\mathrm{m} / \mathrm{min})}{\text { Drilling Rate }}$ \\
\hline \multicolumn{4}{|c|}{ HOLE 144} \\
\hline Drill & 0.57 & & $0.7^{\mathrm{a}}$ \\
\hline Core 1 & $57-65$ & 8.0 & \\
\hline Drill & $\begin{array}{l}65-85 \\
85-104\end{array}$ & & $\begin{array}{l}0.4 \\
0.4\end{array}$ \\
\hline Core 2 & $104-112$ & 8.0 & \\
\hline Drill & $\begin{array}{l}112-123 \\
123-143 \\
143-162\end{array}$ & & $\begin{array}{l}0.8 \\
1.0 \\
1.1\end{array}$ \\
\hline Core 3 & $162-166$ & 2.2 & \\
\hline Drill & $166-213$ & & 0.5 \\
\hline Core 4 & $213-219$ & 3.3 & \\
\hline Drill & $\begin{array}{l}219-227 \\
227-237 \\
237-264\end{array}$ & & $\begin{array}{l}0.4 \\
0.1 \\
0.5\end{array}$ \\
\hline Core 5 & $\begin{array}{l}264-270 \\
270-274 \\
274-292 \\
292-295\end{array}$ & 1.5 & $\begin{array}{r}0.2 \\
0.2 \\
<0.1\end{array}$ \\
\hline Core 6 & $295-298$ & 1.0 & \\
\hline Core 7 & $298-300$ & 0.7 & \\
\hline Drill & $300-324$ & & no data \\
\hline Core 8 & $324-327$ & 3.0 & \\
\hline \multicolumn{4}{|c|}{ HOLE 144A } \\
\hline Drill & $0-20$ & & 1.0 \\
\hline Core 1 & $20-29$ & 7.3 & \\
\hline Drill & $29-38$ & & 0.9 \\
\hline Core 2 & $38-47$ & 9.2 & \\
\hline Drill & $\begin{array}{c}47-94 \\
94-113 \\
113-133 \\
133-140\end{array}$ & & $\begin{array}{l}0.4 \\
0.4 \\
0.6 \\
0.7\end{array}$ \\
\hline Core 3 & $140-149$ & 9.0 & \\
\hline Drill & $\begin{array}{l}149-152 \\
152-171\end{array}$ & & $\begin{array}{l}0.7 \\
0.4\end{array}$ \\
\hline Core 4 & $171-180$ & 1.9 & \\
\hline Core 5 & $180-189$ & 0.6 & \\
\hline Core 6 & $189-197$ & 0.7 & \\
\hline \multicolumn{4}{|c|}{ HOLE 144B } \\
\hline Core 1 & $0-9$ & 9.2 & \\
\hline Core 2 & $10-19$ & 9.1 & \\
\hline Core 3 & $27-36$ & 8.6 & \\
\hline
\end{tabular}

${ }^{\mathrm{a}}$ Artificially slow because of caution necessary for drilling first part of section.

The age diagnostic fossils for Cores 144, 144A and 144B are given in Tables 3, 3A and 3B, respectively.

\section{Foraminifera}

Rich foraminiferal assemblages were found in all cores at this Site. With the exception of the three deepest cores (144-6,7 and 8), they are all predominantly planktonic. The shallowest core (144B-1) contains a rich Oligocene fauna, which is mixed with some Quaternary near the top; Pliocene; and Middle Miocene species. This may be an indication that post-Oligocene sediments are, or were at one time outcropping in the area. The unconformable contact between the Oligocene and the Middle Eocene can be localized in Core 144 A-2, Section 6, between 112 and 120 $\mathrm{cm}$. Similarly, the Cretaceous/Tertiary boundary in Core 144 A-3, Section 5 , is marked by a distinct faunal break at $98 \mathrm{~cm}$, although there is some minor faunal mixing (probably caused by the drilling) above and below this level. The sediment directly above the boundary is late Paleocene in age (Globorotalia pseudomenardii Zone), but a sample about $40 \mathrm{~cm}$ higher contains a few reworked lower Paleocene markers (Globorotalia trinidadensis, $G$. uncinata). This suggests that Lower Paleocene sediments were at one time present in this region and then eroded. Between Cores 144 A-4 and 144 A-5, there is again a sharp lithologic and faunal change which appears to coincide with a minor hiatus (Upper Campanian on Coniacian?Santonian). The faunas of the dark shales of Cores 144 A-5, 144 A-6, and 144.4 are predominantly, or even exclusively, planktonic. The monotonous assemblages consisting of abundant but rather small-sized individuals indicate slightly anomalous conditions, possibly a deficiency in oxygen. Below Core 144-5 with a transitional planktonic-benthonic fauna including Hedbergella washitensis, the hole entered a sandy-silty section containing almost exclusively benthonic assemblages (144-6 to 8 ). The association of benthonic foraminifera with common ostracods, pelecypods, and gastropods indicates a relatively shallow (shelf?) environment of deposition. The fauna shows no evidence of having been transported and redeposited in deep water.

\section{Nannoplankton}

The youngest nannoplankton occurs in the uppermost part of 144 A-1, which belongs to the Upper Miocene, whereas the base of the core is Lower Oligocene. Rich and diversified Lower Oligocene nannofloras occur in 144 B-1, B-2, A-1, B-3 and in the upper part of 144 A-2. The common occurrence of Reticulofenestra hillae in the Sphenolithus predistentus Zone (144 B-1, B-2, A-1) is unusual and may be caused by reworking from Lower Oligocene or Eocene strata. The unconformable contact of Lower Oligocene on Middle Eocene occurs in 144 A-2 between section 6 and the core-catcher. Middle Eocene nannoplankton was also found in 144-1. Four Upper Paleocene zones were recovered, three of them in one (144 A-3) where Upper Paleocene rests on Lower Maestrichtian. The Upper Cretaceous contains common and well-preserved nannoplankton: $144-3$ and $144 \mathrm{~A}-4$ belong to the Lower Maestrichtian to Upper Campanian and 144 A-5 and A- 6 to the Lower Santonian to Upper Turonian. Somewhat poorer Cenomanian nannoplankton occur in 144-4. The Upper Aptian assemblages in 144-6 through 8 are poor and show unusual species compositions. 
TABLE 2

\begin{tabular}{|c|c|c|c|}
\hline \multirow{2}{*}{ 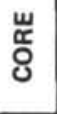 } & \multicolumn{3}{|c|}{ DIAGNOSTIC FOSSILS } \\
\hline & FORAMINIFERA & NANNOPLANKTON & AGE \\
\hline 1 & $\begin{array}{l}\text { Samples of the core contain mainly Recent } \\
\text { planktonic foraminifera and fish debris. } \\
\text { However, in the material recovered from } \\
\text { outside the twisted and damaged core liner } \\
\text { were found small-sized specimens of } \\
\text { Globigerinelloides caseyi, Hedbergella } \\
\text { amabilis, H. planispira, Neobulimina minima, } \\
\text { and Epistomina Zacunosa. } \\
\text { Age: Albian-Cenomanian (probably age } \\
\text { equivalent to the Washita Stage of the U.S. } \\
\text { Gulf Coast). }\end{array}$ & $\begin{array}{l}\text { Poor assemblage including Cretarhabdus coronad- } \\
\text { ventis, Parhabdolithus angustus, Podorhabdus } \\
\text { orbiculofenestrus, Corollithion achyosum, } \\
\text { Corollithion signum, Braarudosphaera bigelowi, } \\
\text { Cylindralithus sp. } \\
\text { Age: Late Albian - Cenomanian. }\end{array}$ & $\begin{array}{l}\text { Late } \\
\text { Albian- } \\
\text { Cenomanian }\end{array}$ \\
\hline
\end{tabular}


TABLE 3

\begin{tabular}{|c|c|c|c|}
\hline \multirow{2}{*}{ 㟧 } & \multicolumn{3}{|c|}{ DIAGNOSTIC FOSSILS } \\
\hline & FORAMINIFERA & NANNOPLANKTON & AGE \\
\hline 1 & $\begin{array}{l}\text { Rich, predominantly planktonic fauna with } \\
\text { Globorotalia crassata, Gr. renai, Gr. } \\
\text { pomeroli, Globigerina senni, Catapsydrax } \\
\text { dissimilis (Sec. 1 to } 3 \text { ), Orbulinoides beck- } \\
\text { manni, Globigerinatheka barri, Chiloguembelina } \\
\text { martini, Nuttallides truempyi. } \\
\text { Age: Middle Eocene, Orbulinoides beckmanni } \\
\text { Zone. }\end{array}$ & $\begin{array}{l}\text { Rich assemblages with Chiasmolithus grandis, } \\
\text { Ch. solitus, Campylosphaera dela, Reticulo- } \\
\text { fenestra umbilica, Discoaster barbadiensis, } \\
\text { D. saipanensis, D. tani nodifer, Sphenolithus } \\
\text { predistentus, S. pseudoradians. } \\
\text { Preservation: E1 - 0l. } \\
\text { Zone: Reticulofenestra umbilica. } \\
\text { Age: Middle Eocene }\end{array}$ & $\begin{array}{l}\text { Middle } \\
\text { Eocene }\end{array}$ \\
\hline 2 & $\begin{array}{l}\text { Rich, predominantly planktonic faunas with } \\
\text { Globorotalia velascoensis, Gr. pseudo- } \\
\text { menardii, Gr. pusilia Zaevigata, Gr. aequa, } \\
\text { Aragonia velascoensis, and Neoflabellina } \\
\text { jarvisi. } \\
\text { Contamination with Eocene and Pliocene } \\
\text { to recent material. } \\
\text { Age: Late Paleocene, Globorotalia pseudo- } \\
\text { menardii Zone. }\end{array}$ & $\begin{array}{l}\text { Abundant nannoplankton including Discoaster } \\
\text { multiradiatus, D. mediosus, Campylosphaera } \\
\text { eodela, Chiasmolithus bidens and Cruciplaco- } \\
\text { lithus eminens. } \\
\text { Preservation: E1 - } 01 \text {. } \\
\text { Zone: Discoaster multiradiatus. } \\
\text { Age: Late Paleocene }\end{array}$ & $\begin{array}{l}\text { Late } \\
\text { Paleocene }\end{array}$ \\
\hline 3 & $\begin{array}{l}\text { Fairly rich faunas with Globotmuncana calici- } \\
\text { formis, Gt. fornicata, Gt. plumerae, Gt. hav- } \\
\text { anensis, Gt. stephensoni, Gt. arca, Gt. } \\
\text { ventricosa, Heterohelix punctulata, H. } \\
\text { ultimatumida, Pseudoguembelina costulata, } \\
\text { Globigerinelloides yaucoensis, G. volutus, } \\
\text { Rugoglobigerina rugosa, R. cfinexacamerata, } \\
\text { Rugotruncana subpennyi, Bolivina incrassata, } \\
\text { Pyramidina szajnochae, Cibicides stephensoni, } \\
\text { Gaudryina laevigata, Bulimina arkadelphiana, } \\
\text { Neoflabellina sp. aff, nwismalis, Aragonia } \\
\text { velascoensis, and Rzehakina epigona lata. } \\
\text { Age: Late Cretaceous, Late Campanian } \\
\text { (or early Maestrichtian?), upper part } \\
\text { of Globotmuncana fornicata - G. stuartiformis } \\
\text { Zone. }\end{array}$ & $\begin{array}{l}\text { Rich and diversified assemblages including } \\
\text { Prediscosphaera cretacea, Cribrosphaerella } \\
\text { ehrenbergi, Microrhabdulus decoratus, Arkhan- } \\
\text { gelskiella cymbiformis, Micula staurophora, } \\
\text { Tetralithus aculeus, Tetralithus obscurus, } \\
\text { Tetralithus pyramidus, Kamptnerius magnificus, } \\
\text { Kamptnerius punctatus, Tetralithus gothicus, } \\
\text { Tetralithus gothicus trifidus, Cylindra } \\
\text { lithus serratus. } \\
\text { Age: Late Campanian - Early Maestrichtian. }\end{array}$ & $\begin{array}{l}\text { Late } \\
\text { Campanian- } \\
\text { Early } \\
\text { Maestrich- } \\
\text { tian }\end{array}$ \\
\hline 4 & $\begin{array}{l}\text { Fairly rich planktonic faunas with } \\
\text { Hedbergella delrioensis, } H \text {. brittonensis, } \\
\text { Heterohelix reussi, } H \text {. moremani, } H \text {. cf. } \\
\text { frizzelli, Globigerinelloides bentonensis, } \\
\text { and G. caseyi. } \\
\text { Age: Late Cenomanian to Early Turonian } \\
\text { (Eagle Ford Stage of U.S. Gulf Coast } \\
\text { stratigraphy). }\end{array}$ & $\begin{array}{l}\text { Common nannoplankton with Cretarhabdus } \\
\text { coronadventis, Prediscosphaera cretacea, } \\
\text { Eiffellithus turriseifeli, Chiastosygus } \\
\text { cuneatus, Zygodiscus exiguus, Podorhabdus } \\
\text { orbiculofenestrus, Broinsonia lata + } \\
\text { Broinsonia bevieri, Corollithion signum, } \\
\text { Staurolithites matalosus. } \\
\text { Age: Cenomanian. }\end{array}$ & $\begin{array}{l}\text { Late } \\
\text { Cenamanian- } \\
\text { Early } \\
\text { Turonian }\end{array}$ \\
\hline 5 & $\begin{array}{l}\text { Fairly rich, mainly planktonic faunas with } \\
\text { Hedbergella washitensis, H. planispira, } \\
\text { H. gautierensis, Globigerinelloides caseyi, } \\
\text { G. bentonensis, Textularia washitensis, } \\
\text { Epistamina tacunosa, Discorbis minutissima, } \\
\text { Neobulimina minima. } \\
\text { Age: Late Albian or Early Cenomanian } \\
\text { (Washita Stage of U.S. Gulf Coast terminology). }\end{array}$ & $\begin{array}{l}\text { Rather poor assemblages including Cretarhabdus } \\
\text { coronadventis, Hayesites albiensis, Parhab- } \\
\text { dolithus angustus, Lithastrinus floralis, } \\
\text { Corollithion achylosum, Staurolithites matalosus, } \\
\text { Braarusosphaera africana. } \\
\text { Age: Late Aptian - Early Albian. }\end{array}$ & $\begin{array}{l}\text { Late } \\
\text { Aptian- } \\
\text { Early } \\
\text { Cenomanian }\end{array}$ \\
\hline
\end{tabular}


TABLE 3 - Continued

\begin{tabular}{|c|c|c|c|}
\hline \multirow{2}{*}{ 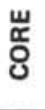 } & \multicolumn{3}{|c|}{ DIAGNOSTIC FOSSILS } \\
\hline & FORAMINIFERA & NANNOPLANKTON & AGE \\
\hline 6 & $\begin{array}{l}\text { Rather poor, mainly benthonic faunas with } \\
\text { Lituola subgoodlandensis, Lenticulina saxo- } \\
\text { cretacea, Textularia washitensis, Epistomina } \\
\text { lacunosa, and Hedbergella washitensis. } \\
\text { Common Ostracoda and remains of Echinoderms. } \\
\text { Age: Late Albian or ?Early Cenomanian } \\
\text { (Washita Stage). }\end{array}$ & $\begin{array}{l}\text { Poor nannoflora with Parhabdolithus angustus, } \\
\text { Lithastrinus floralis, Corollithion achylosum, } \\
\text { Staurolithites matalosus, } \\
\text { Age: Late Aptian - Early Albian. }\end{array}$ & $\begin{array}{l}\text { Late } \\
\text { Aptian- } \\
\text { Early } \\
\text { Cenomanian }\end{array}$ \\
\hline 7 & $\begin{array}{l}\text { Apart from local concentrations of small } \\
\text { mollusks, this core contains a poor benthonic } \\
\text { assemblage with Lituola subgoodlandensis, } \\
\text { Lenticulina saxocretacea, and an Orbitolinid? } \\
\text { (Gen. indet.). Ostracods are fairly common } \\
\text { and include Cytherella gr. parallela/ovata, Anis } \\
\text { ocythere rotundata, and Cythereis spp. (det. } \\
\text { H. J. Oertli). } \\
\text { Age: Late Aptian to Albian (Trinity to lower } \\
\text { Washita Stage of U.S. Gulf Coast). }\end{array}$ & $\begin{array}{l}\text { Rare nannoplankton with Parhabdolithus angustus, } \\
\text { Lithastrinus floralis, Corollithion achylosum, } \\
\text { Staurolithites matalosus, Bracrusosphaera } \\
\text { africana. } \\
\text { Age: Late Aptian - Early Albian. }\end{array}$ & $\begin{array}{l}\text { Late } \\
\text { Aptian- } \\
\text { Albian. }\end{array}$ \\
\hline 8 & $\begin{array}{l}\text { Fairly rich, predominantly benthonic faunas } \\
\text { with Lituola subgoodlandensis, Textularia } \\
\text { rioensis, Spiroplectamina alexanderi, Lingulina } \\
\text { nodosaria, Quinqueloculina sabella, Discorbis } \\
\text { minutissima, Patellina subcretacea, } \\
\text { Guembelitria harrisi, and Hedbergella sp. } \\
\text { (cf. washitensis). Also present are coproliths } \\
\text { (Coptocampylodon lineolatum), small } \\
\text { mollusks, remains of echinoderms, and } \\
\text { ostracods. } \\
\text { Age: Albian (to Late Aptian?), probably } \\
\text { corresponding to the Fredericksburg or lower } \\
\text { Washita Stages of the U.S. Gulf Coast. }\end{array}$ & $\begin{array}{l}\text { Rare nannoplankton with Parhabdolithus angustus, } \\
\text { Lithastrinus floralis, Corollithion achylosum, } \\
\text { Staurolithites matalosus, Braarusosphaera } \\
\text { africana. } \\
\text { Age: Late Aptian - Early Albian. }\end{array}$ & $\begin{array}{l}\text { Late } \\
\text { Aptian- } \\
\text { Albian. }\end{array}$ \\
\hline
\end{tabular}


TABLE 3A

\begin{tabular}{|c|c|c|c|}
\hline \multirow{2}{*}{ 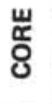 } & \multicolumn{3}{|c|}{ DIAGNOSTIC FOSSILS } \\
\hline & FORAMINIFERA & NANNOPLANKTON & AGE \\
\hline 1 & $\begin{array}{l}\text { Rich, predominantly planktonic faunas with } \\
\text { Globigerina ampliapertura, Globigerina venezuel- } \\
\text { ana, Chiloguembelina cubensis, Cassigerinella } \\
\text { chipolensis, and Pseudohastigerina barbadoensis. } \\
\text { In the upper part of Sec. 1 mixed with a mainly } \\
\text { Pliocene fauna with Globorotalia exilis, Sphaer- } \\
\text { oidinella dehiscens "immatura" etc. } \\
\text { Age: Early Oligocene, Cassigerinella chipo- } \\
\text { lensis/"Hastigerina micra" Zone. }\end{array}$ & $\begin{array}{l}\text { Top of Section 1: Discoaster brouweri, } \\
\text { D. quinqueramus, D. variabilis, Ceratolithus } \\
\text { tricorniculatus. } \\
\text { Zone: Discoaster quinqueramus. } \\
\text { Age: Late Miocene } \\
\text { Lower part of Section 1 through 2: Sphenolithus } \\
\text { predistentus, S. distentus, Reticulofenestra } \\
\text { hillae, Helicopohtosphaera euphratis. } \\
\text { Zone: Sphenolithus predistentus. } \\
\text { Age: Early 01igocene. Sections } 3 \text { through 6: } \\
\text { Sphenolithus predistentus, Reticulofenestra } \\
\text { umbilica, R. hillae, Helicopontosphaera } \\
\text { reticulata, H. euphratis. } \\
\text { Preservation: E1 - 01. } \\
\text { Zone: Helicopontosphaera reticulata. } \\
\text { Age: Early 01igocene. }\end{array}$ & \begin{tabular}{|l} 
Early \\
01igocene
\end{tabular} \\
\hline 2 & $\begin{array}{l}\text { Sec. I to Sec. 6, cm 112: Rich, mainly } \\
\text { planktonic faunas with Globigerina ampliapertura } \\
\text { Gg. venezuelana, Globorotalia opima nana, Gr. } \\
\text { gemma, Catapsydrax dissimilis, Chiloguembelina } \\
\text { cubensis, Cassigerinella chipolensis, } \\
\text { Pseudohastigerina barbadoensis. } \\
\text { Age: Early 01igocene, Cassigerinella Chipolen- } \\
\text { sis/"Hastigerina micra" Zone. } \\
\text { Sec. 6, cm } 120 \text { to Core Catcher: Rich, } \\
\text { predominantly planktonic faunas with } \\
\text { Globorotalia crassata, Gr. renzi, Gr. pomeroli, } \\
\text { Globigerina senni, Globigerinatheka barri, } \\
\text { Hantkenina longispina, Truncorotaloides rohri, } \\
\text { Pseudohastigerina micra, Chiboguembelina cu- } \\
\text { bensis, and Ch. martini. } \\
\text { Age: Late Middle Eocene, Truncorotaloides rohri } \\
\text { Zone. }\end{array}$ & $\begin{array}{l}\text { Sections } 1 \text { through 6: Ericsonia subdisticha, } \\
\text { Reticulofensstra umbilica, Cyclococcolithina, } \\
\text { formosa, Helicopontosphaera reticulata, } H . \\
\text { compacta, Sphenolithus predistentus, S. } \\
\text { tribulosus. } \\
\text { Preservation: E1 - } 01 \text {. } \\
\text { Zone: Ericsonia subdisticha. } \\
\text { Age: Early 01igocene. } \\
\text { Core Catcher: Chiasmolithus grandis, Dis- } \\
\text { coaster barbadiensis, D. saipanensis. } \\
\text { Zone: Reticulofenestra umbilica. } \\
\text { Age: Middle Eocene. }\end{array}$ & $\begin{array}{l}\text { Early } \\
\text { 0ligocene } \\
\text { (lower } \\
\text { part } \\
\text { Middle } \\
\text { Eocene) }\end{array}$ \\
\hline 3 & $\begin{array}{l}\text { Sec. } 1 \text { to Sec. 5, cm 98: Rich, predominantly } \\
\text { planktonic faunas with Globorotalia } \\
\text { velascoensis, Gr. pseudomenardii (rare), } \\
\text { Gr. pusilla pusilia (Sec. } 4 \text { and 5), Gr. } \\
\text { pusilla Zaevigata, Gr. ehrenbergi, Gr. aequa, } \\
\text { Aragonia velascoensis, Bolivinoides } \\
\text { delicatulus, Neoflabellina subreticulata. } \\
\text { In Sec. } 5 \text { also a few apparently reworked } \\
\text { specimens of Globorotalia trinidadensis } \\
\text { and Gr. uncinata. } \\
\text { Age: Late Paleocene, Globorotalia pseudo- } \\
\text { menardii Zone (lower part). } \\
\text { Sec. 5, cm } 98 \text {, to Core Catcher: Rich, } \\
\text { predominantly planktonic faunas with } \\
\text { Globotruncana stuartiformis, Gt. fornicata, } \\
\text { Gt. stephensoni, Gt. havanensis, Gt. aegyptiaca, } \\
\text { Gt. arca, Gt. tricarinata, Heterohelix punctulato } \\
\text { H. ultimatumida, Pseudoguembelina costulata, } \\
\text { Rugoglobigerina hexacamerata, Rugotruncana } \\
\text { subpennyi, Bolivina incrassata, Pyramidina } \\
\text { szajnochae, Marssonella oxycona trinitatensis, } \\
\text { and Aragonia velascoensis. } \\
\text { Age: Late Cretaceous, probably Early } \\
\text { Maestrichtian (upper part of Globotruncana } \\
\text { fornicata-stuartiformis Zone). }\end{array}$ & $\begin{array}{l}\text { This core contains an unconformable contact } \\
\text { of Upper Paleocene on Lower Maestrichtian. } \\
\text { Sections } 1 \text { through 3: Discoaster mohleri, } \\
\text { Ellipsolithus macellus, Zygodiscus sigmoides, } \\
\text { Chiasmolithus consuetus. } \\
\text { Zone: Discoaster mohleri. } \\
\text { Sections } 4 \text { and } 5 \text { (top): Heliolithus kleinpellii, } \\
\text { Zygolithus concinnus, Ellipsolithus macellus, } \\
\text { Fasciculithes tympaniformis. } \\
\text { Zone: Heliolithus kleinpellii. } \\
\text { Section } 5 \text { (40cm - 95cm): Fasciculithes tympani- } \\
\text { formis, Chiasmolithus californicus, Cruciplaco- } \\
\text { lithus eminens. } \\
\text { Zone: Fasciculithes tympaniformis. } \\
\text { Age: Late Paleocene. } \\
\text { Preservation: E1 - O1 (whole core). } \\
\text { Section } 5 \text { (105-107cm) } \\
\text { and Section 6: Kamptnerius punctatus, Tetra- } \\
\text { lithus aculeus, Tetralithus gothicus gothicus, } \\
\text { Kamptnerius magnificus, Tetralithus gothicus } \\
\text { trifidus. } \\
\text { Age: Late Campanian - Early Maestrichtian. }\end{array}$ & \begin{tabular}{|l} 
Late \\
Paleocene \\
\\
\\
Early \\
Maestrich- \\
tian
\end{tabular} \\
\hline
\end{tabular}


TABLE 3A - Continued

\begin{tabular}{|c|c|c|c|}
\hline \multirow{2}{*}{ 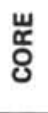 } & \multicolumn{3}{|c|}{ DIAGNOSTIC FOSSILS } \\
\hline & FORAMINIFERA & NANNOPLANKTON & AGE \\
\hline 4 & $\begin{array}{l}\text { Fairly rich faunas with Globotmuncana havanensis } \\
\text { Gt. plummerae, Gt. stephensoni, Gt. linneians. } \\
\text { Heterohelix ultimatumida, H. globulosa, } H . \\
\text { punctulata, Pseudoguembelina costulata, } \\
\text { Globigerinelloides yaucoensis, Planoglobulina } \\
\text { glabrata, Bolivina incrassata, Cibicides } \\
\text { stephensoni, Bulimina taylorensis, Pyramidina } \\
\text { szajnochae, Gyroidina florealis, Neoflabellina } \\
\text { sp. aff. numismalis, Pleurostomella torta, } \\
\text { and Aragonia velascoensis. The faunas are } \\
\text { predominantly bethonic in the lower part of } \\
\text { the core. } \\
\text { Age: Late Campanian or Early Maestrichtian, } \\
\text { Globotruncana fornicata - stuartiformis } \\
\text { Zone. }\end{array}$ & $\begin{array}{l}\text { Rich assemblages with Kamptnerius punctatus, } \\
\text { Tetralithus aculeus, Tetralithus gothicus } \\
\text { gothicus, Kamptnerius magnificus, Tetralithus } \\
\text { gothicus trifidus. } \\
\text { Age: Late Campanian - Early Maestrichtian. } \\
\end{array}$ & $\begin{array}{l}\text { Late } \\
\text { Campanian } \\
\text {-Early } \\
\text { Maestrich- } \\
\text { tian }\end{array}$ \\
\hline 5 & $\begin{array}{l}\text { Rich, predominantly planktonic faunas with } \\
\text { Globotmuncana difformis, Gt. indica, Bedber- } \\
\text { gella amabilis, H. planispira, Hetero- } \\
\text { helix reussi, H. pulchra, H. globulosa, } \\
\text { Valvulineria plumerae, and Bolivina cf. } \\
\text { cretacea. } \\
\text { Age: Coniacian -?Santonian, Marginotruncana } \\
\text { renzi Zone, M. concavata Subzone. }\end{array}$ & $\begin{array}{l}\text { Abundant and well preserved nannoplankton with } \\
\text { the following species Cretarhabdus coronadven- } \\
\text { tis, Eiffellithus turriseiffelli, Chiasto- } \\
\text { aygus cuneatus, Broinsonia lata + } \\
\text { Broinsonia bevieri, Corollithion signum, } \\
\text { Microrhabdulus decoratus, Micula staurophora, } \\
\text { Tetralithus pyramidus, Ahmuellerella octoradiata, } \\
\text { Lithastrinus grilli, Eiffellithus augustus, } \\
\text { Microrhabdulus belgicus. } \\
\text { Age: Late Turonian - Early Santonian }\end{array}$ & $\begin{array}{l}\text { Coniacian } \\
\text {-Santonian } \\
\end{array}$ \\
\hline 6 & $\begin{array}{l}\text { Rich, predominantly planktonic faunas with } \\
\text { Globotmuncana indica, Gt. renzi, Gt. cf. } \\
\text { fornicata, Gt. ventricosa primitiva, Hetero- } \\
\text { helix reussi, H. globulosa, H. cf. frizzelli, } \\
\text { Globigerineiloides asper, Berthelinella? sp., } \\
\text { Lenticulina rotulata, L. cf. taylorensis. } \\
\text { Age: Coniacian, Marginotmucana renzi Zone. }\end{array}$ & $\begin{array}{l}\text { Abundant and well preserved nannoplankton with } \\
\text { the following species Cretarhabdus coronadven- } \\
\text { tis, Eiffellithus turriseiffelli, Chiasto- } \\
\text { zygus cuneatus, Broirsonia lata + } \\
\text { Broinsonia bevieri, Corollithion signum, } \\
\text { Microrhabdulus deciratus, Micula staurophora, } \\
\text { Tetralithus pyramidus, Ahmuellerella octoradiata, } \\
\text { Lithastrinus grilli, Eiffellithus augustus, } \\
\text { Microrhabdulus belgicus. } \\
\text { Age: Late Turonian - Early Santonian }\end{array}$ & $\begin{array}{l}\text { Late } \\
\text { Turonian } \\
\text { Coniacian } \\
\\
\end{array}$ \\
\hline
\end{tabular}


TABLE 3B

\begin{tabular}{|c|c|c|c|}
\hline \multirow{2}{*}{ 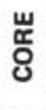 } & \multicolumn{3}{|c|}{ DIAGNOSTIC FOSSILS } \\
\hline & FORAMINIFERA & NANNOPLANKTON & AGE \\
\hline 1 & $\begin{array}{l}\text { Rich, predominantly planktonic faunas with } \\
\text { Globigerina ampliapertura, Gg. venezuelana, } \\
\text { Globorotalia gemma, Catapsydrax dissimilis, } \\
\text { Chiloguembelina cubensis, Cassigerinella chip- } \\
\text { olensis. In Sections } 1 \text { to } 3 \text {, these faunas are } \\
\text { mixed with Quaternary, Pliocene, and Miocene } \\
\text { species such as Globorotalia truncatulinoides, } \\
\text { Gr. miocenica, Gr. tumida, Gr. cultrata, Gr. } \\
\text { exilis, Gr. fohsi peripheroacuta, Globigeri- } \\
\text { noides miber, G. obliquus, Sphaeroidinella } \\
\text { dehiscens, and Sphaeroidinellopsis seminulina. } \\
\text { Age: Early 0ligocene, Globigerina ampliaper- } \\
\text { tura Zone. }\end{array}$ & $\begin{array}{l}\text { Rich nannoflora with Sphenolithus predistentus, } \\
\text { S. distentus, Reticulofenostra hillae, } \\
\text { Helicopontosphaera euphratis. } \\
\text { Preservation: 01. } \\
\text { Zone: Sphenolithus predistentus. } \\
\text { Age: Early 0ligocene }\end{array}$ & $\begin{array}{l}\text { Early } \\
01 \text { igocene }\end{array}$ \\
\hline 2 & $\begin{array}{l}\text { Rich, predominantly planktonic faunas with } \\
\text { Globigerina ampliapertura, Gg. venezuelana, } \\
\text { ug. praebulloides, Gg. pera, Catapsydrax } \\
\text { dissimilis, Chiloguembelina cubensis, } \\
\text { Cassigerinella chipolensis, and Pseu- } \\
\text { dohastigerina barbadoensis. } \\
\text { Age: Early 0ligocene, Cassigerinella chip- } \\
\text { olensis/"Hastigerina micra" Zone. }\end{array}$ & $\begin{array}{l}\text { The same assemblages as in Core B2. } \\
\text { Zone: Sphenolithus predistentus. } \\
\text { Age: Early 01igocene. }\end{array}$ & \begin{tabular}{|l} 
Early \\
01igocene
\end{tabular} \\
\hline 3 & $\begin{array}{l}\text { Rich, predominantly planktonic faunas with } \\
\text { Globigerina ampliapertura, Gg. venesuelana, } \\
\text { Chiloguembelina cubensis, Cassigerinella } \\
\text { chipolensis, Pseudihastigerina barbadoensis. } \\
\text { Age: Early Oligocene, Cassigerinella chipo- } \\
\text { lensis/"Hastigerina micra" Zone. }\end{array}$ & $\begin{array}{l}\text { Diversified assemblages including Ericsonia } \\
\text { subdisticha, Reticulofenestra umbilica, } \\
\text { Cyclococcolithina formosa, Sphenolithus } \\
\text { predistentus, S. tribulosus, Helicoponto- } \\
\text { sphaera reticulata. } \\
\text { Preservation: E1- } 01 \text {. } \\
\text { Zone: Ericsonia subdisticha. } \\
\text { Age: Early 0ligocene }\end{array}$ & $\begin{array}{l}\text { Early } \\
01 \text { igocene }\end{array}$ \\
\hline
\end{tabular}

\section{Organic Microfossils}

By D.O.J. Diederix, R. de Haan and W.O. Tichler ${ }^{2}$

Hole 144

Core 3 Section 2: 137-139 cm:

Barren of sporomorphs, and only a few indeterminate microplankton specimens.

Core 4 Section 2: $27-29 \mathrm{~cm}$ :

No sporomorphs or microplankton recovered.

Core 4 Section 3: 140-142 cm:

Barren of sporomorphs, and microplankton.

Core 5 Section 1: $40-42 \mathrm{~cm}$ :

Rather rich sporomorph flora, containing abundant Classopollis classoides, common Araucariacites australis, Ephedripites sp., (Elaterosporites) Galeacornea klaszi rare (El.) G. verrucata, and Ephedripites spiralis.

Age: Early Cretaceous, Albian to Cenomanian.

Microplankton species found are Coronifera oceanica/ albertii, Odontochitina operculata and Dinopterygium cladoides.

Age: Cretaceous, probably post-Aptian.

\footnotetext{
${ }^{2}$ Koninklijke/Shell Exploratie en Produktie Laboratorium
}

Core 6 Section 1 (bottom):

Sporomorph flora poor in species, with Classopollis classoides, Perotriletes sp., Ephedripites sp., Araucariacites australis, and Galeacornea klaszi.

Age: Possibly Albian to Cenomanian.

Very poor microplankton flora with Trichodinium castaneum.

Age: Late Hauterivian to Campanian.

Core 7 Section 1: 100-102

Rich sporomorph flora containing Ephedra spiralis, Perotriletes sp., Tricolpites sp., Zonalapollenites dampieri, Classopollis classoides, Leptolepidites psarosus, Eucommiidites troedsonii, Ephedripites spp., Galeacornea klaszi, Araucariacites australis, Camarozonosporites cf. insignis, Cicatricosisporites sp. C. $c f$. brevilaesuratus, and $c f$. Raistrickia obtusispina.

Age: Albian to Cenomanian.

Microplankton poorly represented, with Trichodinium castaneum and Dinopterygium cladoides.

Age: Cretaceous, probably post-Aptian.

Core 8 Section 3: $60-62 \mathrm{~cm}$ :

A rather rich sporomorph flora with abundant Classopollis classoides, common Araucariacites australis, Ephedripites sp., (Elaterosporites) Galeocornea klaszi, Trilites sp. (Jardiné \& Magloire 1963 type S. CI. 124), rare 
Eucommiidites troedsonii, Zonalapollenites dampieri and (Elaterosporites) Galeacornea verrucata.

Age: Early Cretaceous, Albian to Cenomanian.

Microplankton species found are Trichodinium castaneum and a specimen belonging probably to Dinopterygium cladoides.

Age: Cretaceous, probably post-Aptian.

\section{Hole 144A}

Core 6 CC:

No sporomorphs recovered and only 1 specimen of the microplankton genus Dinogymnium.

Age: Probably Senonian.

\section{SITE 144 LITHOSTRATIGRAPHIC SUMMARY}

Three holes $(144,144 \mathrm{~A}$, and 144B) were drilled at Site 144 on the outermost edge of Demerara Rise in a water depth of 2957 meters. Depth of penetration was 327, 197 and 36 meters, respectively, for each hole. The following five lithostratigraphic units can be distinguished:

\begin{tabular}{|c|c|c|c|c|}
\hline Unit & Cores & Lithology & $\begin{array}{l}\text { Depth Below } \\
\text { Sea Floor } \\
\text { (m) }\end{array}$ & Age \\
\hline 1 & $\begin{array}{l}\text { B-1, } \\
\text { B-2, } \\
\text { A-1, } \\
\text { B-3, } \\
\text { A-2, } \\
1,2\end{array}$ & $\begin{array}{l}\text { Chalk ooze, } 70- \\
80 \% \text { carbonate } \\
\text { forams nannos, } \\
\text { Radiolarian rich in } \\
\text { Eocene, slightly } \\
\text { siliceous above } \\
\text { and below }\end{array}$ & $0-120(?)$ & $\begin{array}{l}\text { Oligocene- } \\
\text { Paleocene }\end{array}$ \\
\hline 2 & $\begin{array}{l}\text { A-3 } \\
3 \\
\text { A-4 }\end{array}$ & $\begin{array}{l}\text { Zeolitic greenish } \\
\text { gray marl, } 40-65 \% \\
\text { carbonate partly } \\
\text { cemented (calcareous } \\
\& \text { siliceous) pyritic. } \\
\text { Forams nannos, } \\
\text { lower part mostly } \\
\text { carbonate } \\
\text { fragments }\end{array}$ & 120(?)-180 & $\begin{array}{l}\text { Paleocene- } \\
\text { Campanian }\end{array}$ \\
\hline 3 & $\begin{array}{l}\text { A-5, } \\
\text { A-6, } \\
4\end{array}$ & $\begin{array}{l}\text { Zeolite black and } \\
\text { olive marl, } 25-35 \% \\
\text { carbonate much } \\
\text { organic matter } \\
\text { marly LS beds: } \\
40-80 \% \text { carbonate } \\
\text { forams nannos } \\
\text { carbonaceous- } \\
\text { fragments pyrite, } \\
\text { laminae \& varves } \\
\text { (?) glauconite \& } \\
\text { phosphorite in } \\
\text { limestone, forams } \\
\text { (benthonic }> \\
\text { planktonic) filled } \\
\text { with sparry calcite }\end{array}$ & $180-240(?)$ & $\begin{array}{l}\text { Senonian- } \\
\text { Cenomanian }\end{array}$ \\
\hline 4 & 5 & $\begin{array}{l}\text { Olive gray marl, } \\
\text { slightly quartzose, } \\
30-70 \% \text { carbonate, } \\
\text { zeolitic, pyritic, few } \\
\text { forams, nannos }\end{array}$ & $240(?)-280$ & $\begin{array}{l}\text { Early } \\
\text { Turonian to } \\
\text { Late Aptian }\end{array}$ \\
\hline 5 & $6,7,8$ & $\begin{array}{l}\text { Quartzose marlstone, } \\
\text { with shelly limestone } \\
\text { and carbonaceous } \\
\text { clay }\end{array}$ & $280(?)-327+$ & $\begin{array}{l}\text { Late Albian } \\
\text { to Early } \\
\text { Aptian }\end{array}$ \\
\hline
\end{tabular}

UNIT 1 - Chalk Ooze (Cores B-1, B-2, A-1, B-3, A-2, 1, 2)

Unit 1 is about 120 meters thick and is of Oligocene to Paleocene age. It is a marl-chalk ooze with about 70 per cent carbonate material, foraminifera (mainly benthonic) and coccoliths each contributing about equal amounts. Triturated carbonate is a minor component $(<10 \%)$ in the upper half of the unit, but is a major one $(\sim 40 \%)$ in the lower half.

The degree of oxidation decreases downward and is reflected as a color change (pale yellow to pale yellow green to pale green to light green gray) and as a decrease of iron oxide (possibly also manganese oxide) with a corresponding increase of pyrite. Reducing conditions were mostly diagenetic since layering is observed in only one place in Core A-2 whereas mottling and burrowing are ubiquitous. Maximal vertical burrow extent is $3 \mathrm{~cm}$ suggesting that reduction started not far below the sediment surface. Core A2 also contains pyritized radiolarian fragments.

The chalk is siliceous throughout containing in most parts about 7 per cent radiolarians, small opal platelets, and sponge spicules. Within the Eocene cores A-2 and 1, silica contents are higher, especially in 1 where they reach 30 per cent. The lower part of Unit 1 is slightly zeolitic.

Much of Unit 1 consists of alternating soft and firm sediment, and a large proportion of it was too soupy to be cut. Only Cores A-1 and B-3, recovered from 20 to 36 meters, are relatively firm. When sieved, soupy and soft sediments yield clay clasts of several different lithologies: light green gray chalk, green gray chalk, light brownish gray chalk, and yellowish brown zeolitic clay. A layer of this clay, $5 \mathrm{~cm}$ thick, was found in the top of Core A-1 (depth $20 \mathrm{~m}$ ) but may be from anywhere in interval $0-20$ meters as the core barrel was in place when the pipe was washed down. It is not clear to what extent the soft-firm alternations and the mixture of clasts in a soupy matrix are due to drilling.

Sedimentation rates in the Oligocene were $4.5 \mathrm{~m} / \mathrm{my}$ and in the Eocene/Paleocene $6.5 \mathrm{~m} / \mathrm{my}$. Based on paleontological data, a hiatus of about 8 to 9 m.y. was detected at a depth of 46 meters in Core A-2 where Early Oligocene rests on Middle Eocene. Drilling rates ranged from 1.0-0.3 meters/minute in this unit.

\section{UNIT 2 - Zeolitic Greenish Gray Marl (Cores A-3, 3, A-4)}

Unit 2 is about 60 meters thick and ranges in age from Paleocene to Maestrichtian. This unit consists largely of finely and densely mottled greenish gray and light greenish gray high-grade marl (30-55\% carbonate). It is generally indurated and, in places, lithified. Drilling rates averaged just less than 1 meter/minute in this unit.

The carbonate is provided by foraminifera, coccoliths, and triturated fragments in about equal parts, A $30 \mathrm{~cm}$ thick limestone bed made of calcisilt occurs near the top of the unit (A-3). Only traces of radiolarians are present. Zeolites are abundant (5-15\%). About 2 per cent of pyrite occurs throughout the unit, occasionally as pyrite lenses. A few percent of terrigenous minerals, mainly quartz, appear toward the base of the unit (A-4), as do silicified marly claystones about $10-15 \% \mathrm{~cm}$ thick. Recovery was poor in this part of the section. 


\section{DRILLING SPEED-METERS/MIN.}

LITHOSTRATIGRAPHIC

UNIT NO.

CORE

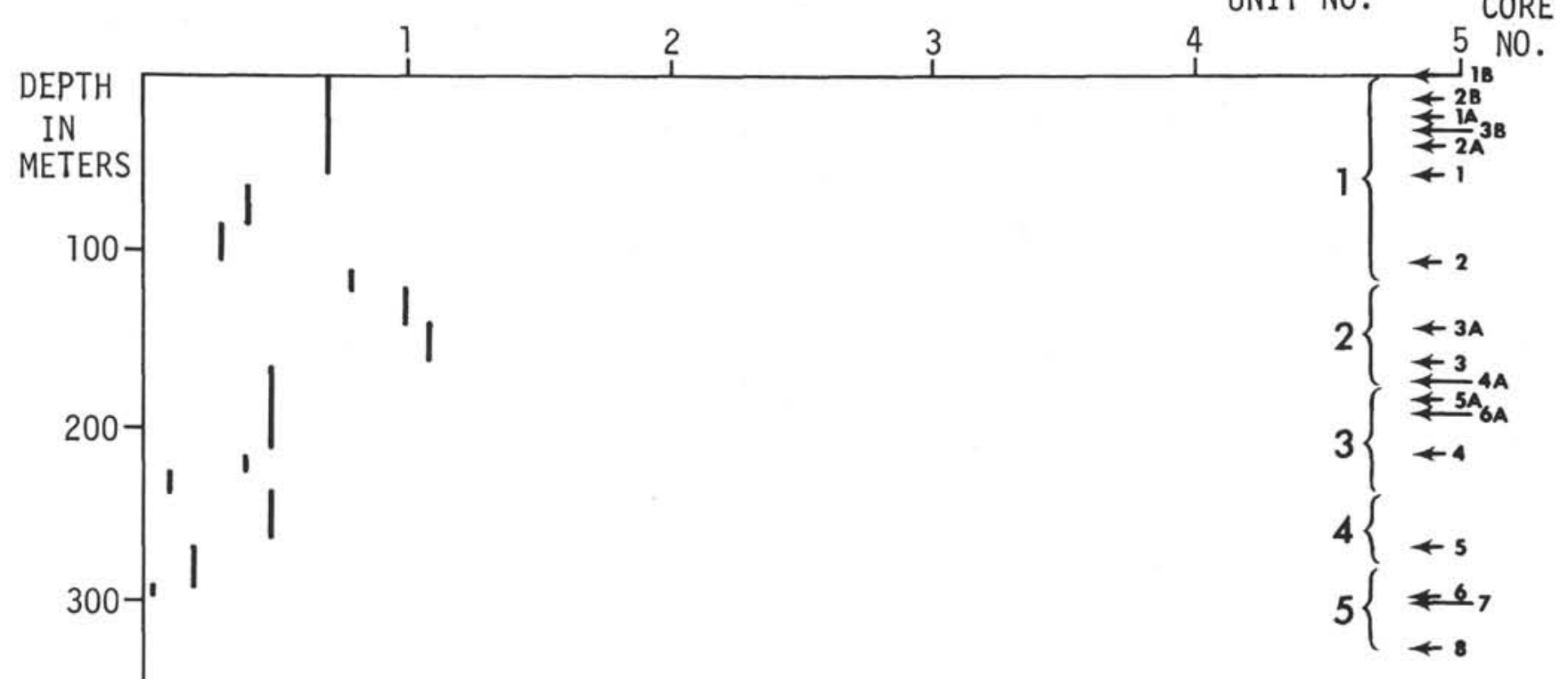

400

Figure 6. Summary of drilling and coring at Site 144.

The sediment is well burrowed (maximum vertical extent $2 \mathrm{~cm}$ ) except in some parts of A-4 (base of unit). The pyrite formation appears to be early diagenetic rather than synsedimentary. Faint banding in A3 (top of unit), between lighter and darker greenish gray, suggests redeposition processes. The lighter material contains more zeolite, the darker is richer in carbonate.

A hiatus of about 8 my was detected, based on paleontological data, at a depth of 147 meters. In the early Maestrichtian sedimentation rates were $5 \mathrm{~m} / \mathrm{my}$.

\section{UNIT 3 - Zeolitic Black and Olive Marl (Cores A5, A6, 4)}

Unit 3 is about 60 meters thick and is Senonian to Cenomanian in age. It is characterized by a sequence of dark shales and clay with interrelated lime and marlstone beds several $\mathrm{cm}$ thick. Recovery is poor and this fact may distort the proportions of the various sediment types. They are, in order of decreasing abundance:

1) Olive black and olive finely laminated calcareous carbonaceous clay and shale, which contains occasional lighter colored calcareous laminae and layers. The sediment consists of roughly equal parts of carbonate $(\sim 30 \%)$, organic matter, and clay, with about 10 to 20 per cent zeolite minerals. The olive clay contains distinctly less organic matter and more clay (Core 4 , base of unit), The carbonate consists of forams, coccoliths, and triturated fragments in about equal amounts. Pyrite is present throughout and terrigenous components occur in traces.

2) Foram rich olive carbonaceous limestone has a sparry calcite cement which also fills the foram tests. The limestone $(80 \%)$ is rich in clay and organic matter. Some zeolite and a few radiolarians are also present.

3) Grayish green glauconitic and phosphoritic marlstone contains common forams (mainly benthonic), pyrite and limonite and has a sparry cement. A thin section shows 25 per cent fresh green glauconite, lobate grains, partly calcitized, and 25 per cent phosphate minerals (collophane, apatite, dahlite, fish teeth). Terrigenous minerals occur in traces.

Sedimentation rates ranged from 3 to $4 \mathrm{~m} / \mathrm{my}$. Drilling rates in this unit were $>0.5 \mathrm{~meter} /$ minute.

\section{UNIT 4 - Olive Green Marl (Core 5)}

Unit 4, Late Aptian to Early Cenomanian in age, is about 40 meters thick. Only 1.5 meters of sediment was recovered in this unit. Like Unit 3, this marl shows laminations and is slightly pyritic. The bulk of the carbonate $(\sim 50 \%)$ is provided by triturated "flour" rather than by whole fossil tests. Unit 4 has distinctly more quartz than Unit 3, about 5 to 10 per cent. Zeolite is present also. Sediments accumulated at about $10 \mathrm{~m} / \mathrm{my}$. Drilling rates were much less than 0.5 meter/minute.

UNIT 5 - Quartzose Marlstone with Shelly Limestone and Carbonaceous Clay (Cores 6, 7, 8)

This unit is at least 50 meters thick and is Late Aptian to Early Cenomanian in age. The sediment types recorded in Unit 5 are (from top to bottom) shelly limestone, silty marlstone, slightly quartzose zeolitic (?) clay, slightly quartose carbonaceous clay, coquina marl, quartzose calcareous silty calcareous claystone, and mudstone.

Carbonate content averages 30 per cent, with a range of from 10 per cent (carbonaceous clay) to 60 per cent (in part shelly limestone). All types have in common: (1) a relative high quartz content $(15-40 \%)$; (2) organic matter (few \%); (3) pyrite (few \%); and (4) a sizable proportion of the carbonate present being provided by mollusk shell fragments. Nine-tenths of the recovered unit is burrowed 
and mottled. Radiolarians are rare and are largely pyritized or (?) calcitized.

The most distinctive lithologies are those of the shelly limestone in Core 6 and the quartzose and pyritic carbonaceous clay in Core 7. Most of the other lithologies are intermediate between these two types.

The shelly limestone is dark greenish gray to olive gray, mottled and burrowed, and contains large (1-5 cm long) fragments of gastropods and pelecypods. A thin section shows 55 per cent $\mathrm{CaCO}_{3}$ (5\% shells, $50 \%$ micrite and sparry cement), 25 per cent very angular quartz, 5 per cent feldspar, 5 per cent micas, 3 per cent pyrite, 7 per cent clay minerals, and a trace of chert (?).

The carbonaceous clay is dark greenish gray, homogenous with faint layering in places, and consists of clay minerals and organic matter with 20 per cent quartz, 3 per cent mica, 15 per cent pyrite, and 10 per cent carbonate (shell fragments, also forams). At least one claystone bed appears intraclastic. Near the base (Core 8 , bottom) a slightly more calcareous clay is olive black and irregularly laminated.

Sedimentation rates were about $3 \mathrm{~m} / \mathrm{my}$.

\section{Sedimentation Trends}

1. Oxygenation: Except for the uppermost 10 meters, the whole section drilled contains pyrite and has greenish color, indicating reducing conditions within the sediment. Pyrite and organic matter contents are highest in the lowermost unit and laminated black sediments occur in places below 200 meters. The bulk of the sequence shows intense mottling and burrowing. A general increase of oxygenation from bottom to top of the section appears indicated, especially in post-Cretaceous sediments.

2. Carbonate Content: The entire section is calcareous with marl predominating in the Cretaceous and low-grade chalk in the Early Tertiary. At the bottom of the section, much of the carbonate is provided by mollusk shells and by recrystallized calcite, by triturated calcite "flour" in about equal proportions (Units $3,2,1$ ) and finally by forams and nannos with minor "flour" (Unit 1, upper part).

3. Opal Content: Below 150 meters, within the Cretaceous, siliceous fossils are very rare, except for occasional pyritized and calcitized (?) radiolarian fragments. Silicacemented marlstones appear in Core A3 (Unit 2) and from there on up siliceous fossils, especially radiolarians. There are also sponge spicules and unidentified opal platelets, are quite common (5\% and more) with a distinct maximum (30\% siliceous) in Core 1 (Unit 1, Mid-Eocene).

4. Zeolite Content: Zeolites are common (5\%) in Units 2,3 , and 4 , but are much less prominent at the base (Unit 5) and at the top (Unit 1) of the sequence. Maximum zeolite contents (10-15\%) are found within Paleocene/Late Cretaceous sediments between about 140 to 230 meters.

5. Terrigenous Influx: Greatest contents of quartz and associated mica are found at the base of the section $(30-50 \%)$ (Unit 5$)$. Contents decrease rapidly upwards (5\%, Unit $4 ; 1 \%$ lower part of Unit 3) and are virtually absent above' 200 meters (post-Cenomanian).

6. Non-Terrigenous Redeposition: Evidence of redeposition is present throughout the sequence. Within Unit 5 , the interbedding of shell beds and clay layers suggests introduction of shallow water carbonate debris into a carbonate-poor clay sequence. A somewhat similar situation appears in Unit 4. Here the carbonate consists of finely triturated clasts which are concentrated in light colored layers $(70 \%)$ that alternate with darker layers (35\% carbonate) on a scale of a few millimeters to $2 \mathrm{~cm}$. Boundaries are distinct, sometimes sharp. Similarly, Unit 3 consists of a calcareous clay sequence with apparently redeposited lime and marl beds that are rich in benthonic forams and (in places) in glauconite and phosphorite. Alternations between carbonate and zeolite-rich bands in Unit 2 again suggest redeposition processes, as do lithified layers rich in carbonate. The mixture of different kinds of ooze clasts within a soupy matrix in Unit 1 also may be due to redeposition processes, although the possible effects of drilling on these disturbances are not clear.

\section{SITE 144 PHYSICAL AND CHEMICAL PROPERTIES}

The physical and chemical properties of the sediments cored at Site 144 suggest a three-fold division of the section: 1) upper unit (0-112 m) consisting of chalk oozes, Oligocene to Late Paleocene in age; 2 ) middle unit (140-220 $\mathrm{m}$ ) consisting of calcareous mudstones and carbonaceous clays Early Paleocene to Turonian in age; and 3) lower unit (264-327 m), quartzose calcareous mudstones, marls, and limestones, more indurated than the middle unit, Albian and Aptian in age. The upper unit corresponds to Unit I of the lithologic summary; the middle unit, to Units II and III, and the lower unit to Units IV and V.

\section{Wet-Bulk Density and Porosity}

GRAPE measurements (Table 4) of the bulk density $(\mathrm{gm} / \mathrm{cc})$ and porosity $(\%)$ suggest the three-fold division noted above correlates well with detailed changes in lithology. Bulk densities of relatively undisturbed parts of the chalk oozes of the upper unit fall mainly between 1.3 and 1.6, with mean values increasing slightly with depth. Porosities average about 70 per cent. Lower bulk densities and higher porosities correlate with softer sediments which were probably disturbed by drilling.

Bulk densities average about 1.75 , and the porosities run 65 to 70 per cent for the middle unit of calcareous mudstones and zeolitic carbonaceous clays. Deviations from the mean correspond to beds of less compacted clay and beds of cemented marly limestone.

In the lower unit ( 265 to $327 \mathrm{~m}$ ) bulk density runs 1.7 to 1.9 ; porosity ranges from 20 to 40 per cent.

\section{Water Content}

Water content decreases with depth from about 40 per cent at the top to about 16 per cent at 300 meters.

\section{Natural Gamma}

Variation in the natural gamma counts correlates well with the detailed lithology. Except for high counts in the topmost two meters of the section (common in many oceanic deposits), gamma readings are low (50-600) in the oozes of the upper unit. In the middle unit, gamma counts range from 500 to 1300 in the calcareous mudstones and from 1100 to 2600 in the carbonaceous clays. The variations appear to correlate with the zeolite content of 
TABLE 4

Summary of Density, Porosity and Water Content Data for Sites 143 and 144

\begin{tabular}{|c|c|c|c|c|c|c|c|c|c|}
\hline \multirow[b]{2}{*}{ Hole } & \multirow[b]{2}{*}{ Core } & \multirow[b]{2}{*}{ Section } & \multicolumn{3}{|c|}{ GRAPE } & \multicolumn{4}{|c|}{ Sample Sediment } \\
\hline & & & $\begin{array}{l}\text { Septh Below } \\
\text { Sea Floor } \\
\text { (m) }\end{array}$ & $\begin{array}{l}\text { Density } \\
(\mathrm{gm} / \mathrm{cc})\end{array}$ & $\begin{array}{c}\text { Porosity } \\
(\%)\end{array}$ & $\begin{array}{l}\text { Depth Below } \\
\text { Sea Floor } \\
\text { (m) }\end{array}$ & $\begin{array}{l}\text { Water } \\
\text { Content } \\
(\%)\end{array}$ & $\begin{array}{l}\text { Density } \\
(\mathrm{gm} / \mathrm{cc})\end{array}$ & $\begin{array}{c}\text { Porosity } \\
\text { (\%) }\end{array}$ \\
\hline $\begin{array}{l}143 \\
143\end{array}$ & $\begin{array}{l}1 \\
1\end{array}$ & $\begin{array}{l}1 \\
2\end{array}$ & $\begin{array}{l}14.75 \\
16.25\end{array}$ & $\begin{array}{l}1.61 \\
1.60\end{array}$ & $\begin{array}{l}48 \\
49\end{array}$ & - & $\overline{-}$ & - & - \\
\hline 144 & 1 & 1 & 57.75 & 1.38 & 69 & - & 38.95 & 1.430 & 55.71 \\
\hline 144 & 1 & 2 & 59.25 & 1.46 & 62 & - & 34.52 & 1.460 & 50.40 \\
\hline 144 & 1 & 3 & 60.75 & 1.42 & 66 & - & 37.50 & 1.407 & 52.76 \\
\hline 144 & 1 & 4 & 62.25 & 1.48 & 60 & - & - & - & - \\
\hline 144 & 1 & 5 & 63.75 & 1.47 & 61 & - & - & - & - \\
\hline 144 & 1 & 6 & 65.25 & 1.50 & 58 & - & - & - & - \\
\hline 144 & 2 & 1 & 104.75 & 1.35 & 71 & - & - & - & - \\
\hline 144 & 2 & 2 & 106.25 & 1.41 & 66 & - & - & - & - \\
\hline 144 & 2 & 3 & 107.75 & 1.30 & 76 & - & - & - & - \\
\hline 144 & 2 & 4 & 109.25 & 1.31 & 75 & - & - & - & - \\
\hline 144 & 2 & 5 & 110.75 & 1.24 & 81 & - & - & - & - \\
\hline 144 & 2 & 6 & 112.25 & 1.40 & 68 & - & - & - & - \\
\hline 144 & 3 & 1 & 162.75 & 1.53 & 56 & - & - & - & - \\
\hline 144 & 3 & 2 & 164.25 & 1.54 & 55 & - & 20.39 & 1.578 & 32.17 \\
\hline 144 & 4 & 2 & 215.25 & 1.48 & 60 & - & - & - & - \\
\hline 144 & 4 & 3 & 216.75 & 1.43 & 65 & - & - & - & - \\
\hline 144 & 5 & 1 & 264.75 & 1.91 & 22 & - & 14.41 & 1.610 & 23.20 \\
\hline 144 & 6 & 1 & 295.75 & 1.72 & 39 & - & - & - & - \\
\hline 144 & 7 & 1 & 298.75 & 1.82 & 30 & - & 15.53 & 1.854 & 28.80 \\
\hline $144 \mathrm{~A}$ & 1 & 1 & 20.75 & 1.23 & 78 & - & - & - & - \\
\hline $144 \mathrm{~A}$ & 1 & 2 & 22.25 & 1.48 & 51 & 21.64 & 40 & 1.36 & 55 \\
\hline $144 \mathrm{~A}$ & 1 & 3 & 23.75 & 1.53 & 45 & 23.14 & 36 & 1.49 & 53 \\
\hline $144 \mathrm{~A}$ & 1 & 4 & 25.25 & 1.54 & 45 & 24.64 & 35 & 1.52 & 53 \\
\hline $144 \mathrm{~A}$ & 1 & 5 & 26.75 & 1.58 & 40 & 26.14 & 34 & 1.45 & 49 \\
\hline $144 \mathrm{~A}$ & 2 & 1 & 38.75 & 1.51 & 49 & - & - & - & - \\
\hline $144 \mathrm{~A}$ & 2 & 2 & 40.25 & 1.40 & 60 & - & - & - & - \\
\hline $144 \mathrm{~A}$ & 2 & 3 & 41.75 & 1.37 & 63 & - & - & - & - \\
\hline $144 \mathrm{~A}$ & 2 & 4 & 43.25 & 1.59 & 40 & 42.64 & 35 & 1.46 & 50 \\
\hline $144 \mathrm{~A}$ & 2 & 5 & 44.75 & 1.59 & 41 & 44.24 & 40 & 1.44 & 57 \\
\hline $144 \mathrm{~A}$ & 2 & 6 & 46.25 & 1.60 & 39 & - & - & - & - \\
\hline $144 \mathrm{~A}$ & 3 & 1 & 140.75 & 1.75 & 23 & 140.14 & 27 & 1.57 & 42 \\
\hline $144 \mathrm{~A}$ & 3 & 2 & 142.25 & 1.84 & 14 & - & - & - & - \\
\hline $144 \mathrm{~A}$ & 3 & 3 & 143.75 & 1.73 & 26 & 143.14 & 19 & 1.56 & 30 \\
\hline $144 \mathrm{~A}$ & 3 & 4 & 145.25 & 1.85 & 12 & 144.61 & 18 & 1.37 & 25 \\
\hline $144 \mathrm{~A}$ & 3 & 5 & 146.75 & 1.82 & 16 & - & - & - & - \\
\hline $144 \mathrm{~A}$ & 3 & 6 & 148.25 & 1.74 & 24 & 147.64 & 28 & 1.62 & 46 \\
\hline $144 \mathrm{~A}$ & 4 & 1 & 171.75 & 1.27 & 74 & - & - & - & - \\
\hline $144 \mathrm{~A}$ & 4 & 2 & 173.25 & 1.64 & 34 & 172.69 & 25 & 1.58 & 40 \\
\hline $144 \mathrm{~A}$ & 6 & 1 & 189.75 & 1.32 & 69 & - & - & - & - \\
\hline 144B & 1 & 1 & 0.75 & 1.44 & 67 & - & - & - & - \\
\hline 144B & 1 & 2 & 2.25 & 1.48 & 64 & - & - & - & - \\
\hline 144B & 1 & 3 & 3.75 & 1.51 & 62 & - & - & - & - \\
\hline 144B & 1 & 4 & 5.25 & 1.53 & 60 & 4.64 & 41 & 1.53 & 63 \\
\hline 144B & 1 & 5 & 6.75 & 1.51 & 62 & - & - & - & - \\
\hline 144B & 1 & 6 & 8.25 & 1.57 & 57 & 7.64 & 39 & 1.49 & 58 \\
\hline 144B & 2 & 1 & 10.75 & 1.54 & 55 & 10.37 & 40 & 1.47 & 59 \\
\hline 144B & 2 & 2 & 12.25 & 1.46 & 62 & - & - & - & - \\
\hline 144B & 2 & 3 & 13.75 & 1.47 & 61 & - & - & - & - \\
\hline 144B & 2 & 4 & 15.25 & 1.49 & 59 & - & - & - & - \\
\hline 144B & 2 & 5 & 16.75 & 1.49 & 59 & - & - & - & - \\
\hline 144B & 2 & 6 & 18.25 & 1.50 & 58 & - & - & - & - \\
\hline 144B & 3 & 2 & - & - & - & 28.65 & 39 & 1.48 & 58 \\
\hline 144B & 3 & 3 & - & - & - & 30.14 & 38 & 1.55 & 59 \\
\hline 144B & 3 & 4 & - & - & - & 31.64 & 33 & 1.57 & 52 \\
\hline 144B & 3 & 6 & - & - & - & 34.64 & 40 & 1.54 & 62 \\
\hline
\end{tabular}


the beds. The highest counts ( 2300 and over) correspond to local beds with a zeolite content as high as 20 per cent. In the lower unit of quartz-bearing limestones and calcareous mudstones, gamma counts fall to between 500 and 1300 , which is similar to those of the less indurated mudstones of the middle unit.

\section{Chemical Properties}

Salinities range from 34.7 to 46.2 per cent (see Table 5). Values under 40 are confined to the upper unit of oozes, and the highest values are found in the middle unit.

The $\mathrm{pH}$ values are alkaline in the upper and lower units. Values are slightly acidic ( 6.88 to 6.96 ) in the middle unit, with only one exception ( 7.05 in Core $5 \mathrm{~A}$ ). This zone corresponds to the zone of highest salinity and more importantly, to those sections of core that contained noticeable amounts of $\mathrm{H}_{2} \mathrm{~S}$.

\section{SITES 143 and 144 DISCUSSION AND CONCLUSIONS}

Drilling at Sites 143 and 144 has confirmed the observation, based on seismic profiles and piston core and dredge samples taken by earlier workers, that progressively older rocks crop out down the steep northern flank of the Demerara Rise. Site 144 was drilled just below the relatively flat upper surface of the Rise and recovered Oligocene material at the sea floor. Also included within this surface core are some fragments of Neogene calcareous sediments which have presumably slumped down the slope from higher up on the plateau. Site 143 was situated further down the flank of the Rise, and Cretaceous material was recovered at the sea floor.

The oldest material was recovered in Site 144 at a depth of 327 meters. It is dated as Late Aptian/Early Albian on the basis of nannoplankton, and foraminifera, and as Albian/Cenomanian on the basis of the sporomorph flora. This sample is the same age as material from the one surface core, recovered in Site 143 which was located at a depth 544 meters deeper than Site 144. The age and depth relationship of these two samples suggest that the attitude of sediment layers is not horizontal, but that they dip slightly, partially draping the slope. Additional evidence for a downslope dip of the older beds is seen in the seismic

TABLE 5

Chemical Property Measurements on Samples from Site 144

\begin{tabular}{|c|c|c|c|c|c|c|c|}
\hline \multirow[b]{2}{*}{ Hole } & \multirow[b]{2}{*}{ Core } & \multirow[b]{2}{*}{ Section } & \multicolumn{2}{|c|}{$\begin{array}{l}\text { Sample } \\
\text { Interval } \\
(\mathrm{cm})\end{array}$} & \multirow[b]{2}{*}{$\mathrm{pH}$} & \multirow[b]{2}{*}{ Eh } & \multirow{2}{*}{$\begin{array}{l}\text { Salinity } \\
(\% \circ)\end{array}$} \\
\hline & & & Top & Bottom & & & \\
\hline 144 & $\begin{array}{l}1 \\
3 \\
4 \\
5\end{array}$ & $\begin{array}{c}6 \\
2 \\
3 \\
\text { CC }\end{array}$ & $\begin{array}{r}0.0 \\
140.0 \\
0.0\end{array}$ & $\begin{array}{r}5.0 \\
145.0 \\
6.0\end{array}$ & $\begin{array}{l}7.38 \\
6.96 \\
6.90 \\
7.06\end{array}$ & $\begin{array}{r}+37 \\
+141 \\
+120 \\
+188\end{array}$ & $\begin{array}{l}36.8 \\
40.2 \\
46.2 \\
41.8\end{array}$ \\
\hline $144 \mathrm{~A}$ & $\begin{array}{l}1 \\
2 \\
3 \\
4 \\
5 \\
6\end{array}$ & $\begin{array}{c}2 \\
6 \\
4 \\
\mathrm{CC} \\
\mathrm{CC} \\
\mathrm{CC}\end{array}$ & $\begin{array}{l}0.0 \\
0.0 \\
0.0\end{array}$ & $\begin{array}{r}10.0 \\
0.0 \\
5.0\end{array}$ & $\begin{array}{l}7.51 \\
7.42 \\
6.96 \\
6.88 \\
7.05 \\
6.91\end{array}$ & $\begin{array}{r}+176 \\
+132 \\
+169 \\
+172 \\
+196 \\
+199\end{array}$ & $\begin{array}{l}36.3 \\
36.3 \\
41.2 \\
44.0 \\
45.1 \\
45.6\end{array}$ \\
\hline 144B & $\begin{array}{l}1 \\
2 \\
3\end{array}$ & $\begin{array}{l}6 \\
5 \\
6\end{array}$ & $\begin{array}{l}0.0 \\
0.0 \\
0.0\end{array}$ & $\begin{array}{l}8.0 \\
6.0 \\
8.0\end{array}$ & $\begin{array}{l}7.40 \\
7.32 \\
7.75\end{array}$ & $\begin{array}{r}+192 \\
+87 \\
+119\end{array}$ & $\begin{array}{l}34.7 \\
35.8 \\
35.8\end{array}$ \\
\hline
\end{tabular}

profiles (see Figure 4). However, much of the material recovered at Site 143 is redeposited and the validity of the stratigraphic date there is in question. The water depth of the Shell piston core, dated as Barremian/Aptian on sporomorphs, was 3480 meters, 13 meters shallower than Site 143.

All seismic profiles across the top of the Demerara Rise show a pronounced reflector at 400 to 500 meters sub-bottom, and on the Shell records this reflector defines a pronounced angular unconformity. The surface manifestation of this feature may be a change in slope or narrow terrace part way down the northern flank of the Demerara Rise. Although Site 144 was selected just above such a terrace, no conclusive correlation can be made between the recovered cores and the unconformity surface as the Challenger was unable to obtain any seismic records over the steep slope of the Rise at the precise site location.

Three hiatuses, each of about $8 \mathrm{my}$, were detected in the cored section. These were in the Upper Eocene at 46 meters and at the Cretaceous/Tertiary boundary at 147 meters; neither of these hiatuses coincide with major lithologic changes. The other hiatus at 180 meters, detected between Upper Campanian and Lower Santonian sediments, does coincide with a lithologic change.

The Tertiary/Cretaceous unconformity appears to be an erosional feature as sediments immediately above it contain a Late Paleocene fauna, but higher up there are reworked Early Paleocene marker-fossils suggesting the Lower Paleocene sediment was present at one time but later removed by erosion.

On the basis of water depth, the inferred position of Site 144 is plotted on the seismic record in Figure 2 and the composite diagram in Figure 5. The depth to the unconformity surface at the site location is 0.32 second. The lowermost lithostratigraphic Unit (5) was deposited in shallow water in contrast to the overlying units. Although the depth to the top of the unit is not well defined, it lies somewhere between 270 and 295 meters. If we assume the boundary lies near 280 meters and that this level corresponds with the unconformity, then an average internal velocity of $1.75 \mathrm{~km} / \mathrm{sec}$ is obtained for the overlying units. This velocity is reasonable considering the lithologies encountered.

We would anticipate that such an angular unconformity would be marked by a recognizable hiatus in the sedimentary record. The uncertainty in the paleontologic ages assigned to Units 4 and 5 leaves the question of a possible hiatus open.

The sedimentation record indicates that Site 144 has been on a subsiding terrace edge since Cretaceous times. The total amount of subsidence since the Albian is nearly 3000 meters. Slumping and bottom current action have probably been active along the edge of the terrace throughout this time and could account for the observed hiatuses. Seismic profiles (Figure 2) down the northern slope of the Demerara Rise indicate large slumped masses probably due to down faulting of the terrace edge.

The history of deposition inferred for Site 144 is as follows: During Cretaceous time, deposition took place in a marginally oxygenated environment that alternated between aerobic and anaerobic conditions and favored the 
preservation of organic matter. This carbonaceous material may have been partly introduced from elsewhere. Planktonic productivity was apparently small in the overlying waters as there was little delivery to the sea floor of calcareous or siliceous plankton. The relatively high carbonate content present is apparently due to an influx of shells and reworked limestone from upslope. This influx is derived from a successively deeper source as mollusk shells and fragments appear first, and are followed by triturated carbonate, with increasing pelagic influence. The terrigenous contribution was at a maximum before the introduction of the mollusk shell beds and decreased upward. The glauconitic and phosphoritic limestone in Core A5 formed not long after the terrigenous supply ceased altogether but before the pelagic component had become very strong in the resediments. Derivation from an intermediate position between shelf and upper slope, perhaps from a terrace with little clastic sedimentation, appears indicated for the glauconite and phosphoritic limestone. The resediments are intercalated into an olive and black clay sequence that probably accumulated on a subsiding site with increasing zeolite at successively greater depths.

If the picture of a sinking source area of resediments and a sinking site of deposition is correct, one might visualize a series of terraces breaking off from the continent, one after the other and with Site 144 becoming farther and farther removed from the shelf. Intermediate terraces intervene and trap any shallow water sediment that moves downslope.
In this interpretation, the depth facies during the Cretaceous were as follows (shallow to deep): terrigenous mud, mollusk shells, calcite debris and/or recrystallized limestone zone rich in benthonic forams, glauconite and phosphorite zone (possibly on elevated or terraced parts), - unknown facies interval (organic-rich zone?)-pyritic olive clay, zeolitic olive clay with pyrite.

A fundamental change of sedimentation took place in the beginning of the Tertiary. Fertility increased, and opal began to be preserved. Zeolites became rare, possibly due to dilution with other components or possibly due to a change in the chemistry of interstitial waters. Calcareous nannoplankton and planktonic foraminifera began to dominate the carbonate contribution. The sediments are well oxygenated in the upper few centimeters of the sea floor as shown by burrowing. Reducing conditions appear at greater depth within the sediment, and lead to minor pyrite formation. As mentioned above, much of the sediment drilled may be redeposited.

\section{REFERENCES}

Fox, P. J., Heezen, B. C. and Johnson, G. L., 1970. Jurassic sandstone from the tropical Atlantic, Science, 170, 1402-1404.

Lowrie, A. and Escowitz, E. (Eds.), 1969. Kane 9, U.S. Naval Oceanographic Office. 

SITE 144-SUMMARY

CORED INTERVALS $\underline{\text { CORE }} \quad \underline{\text { LITHOLOGY }}$ CORE $\quad \underline{\text { LITHOLOGY }}$

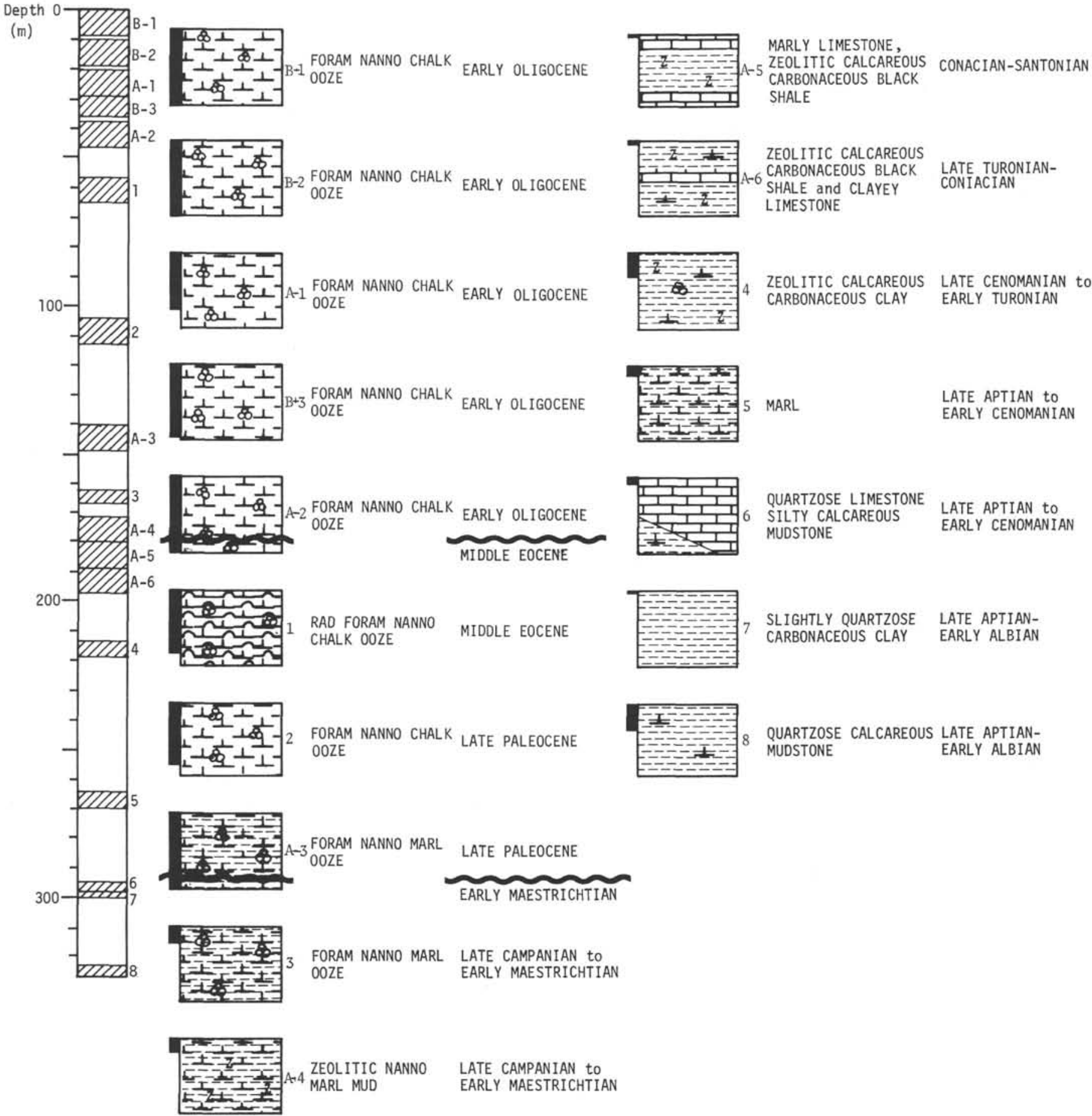




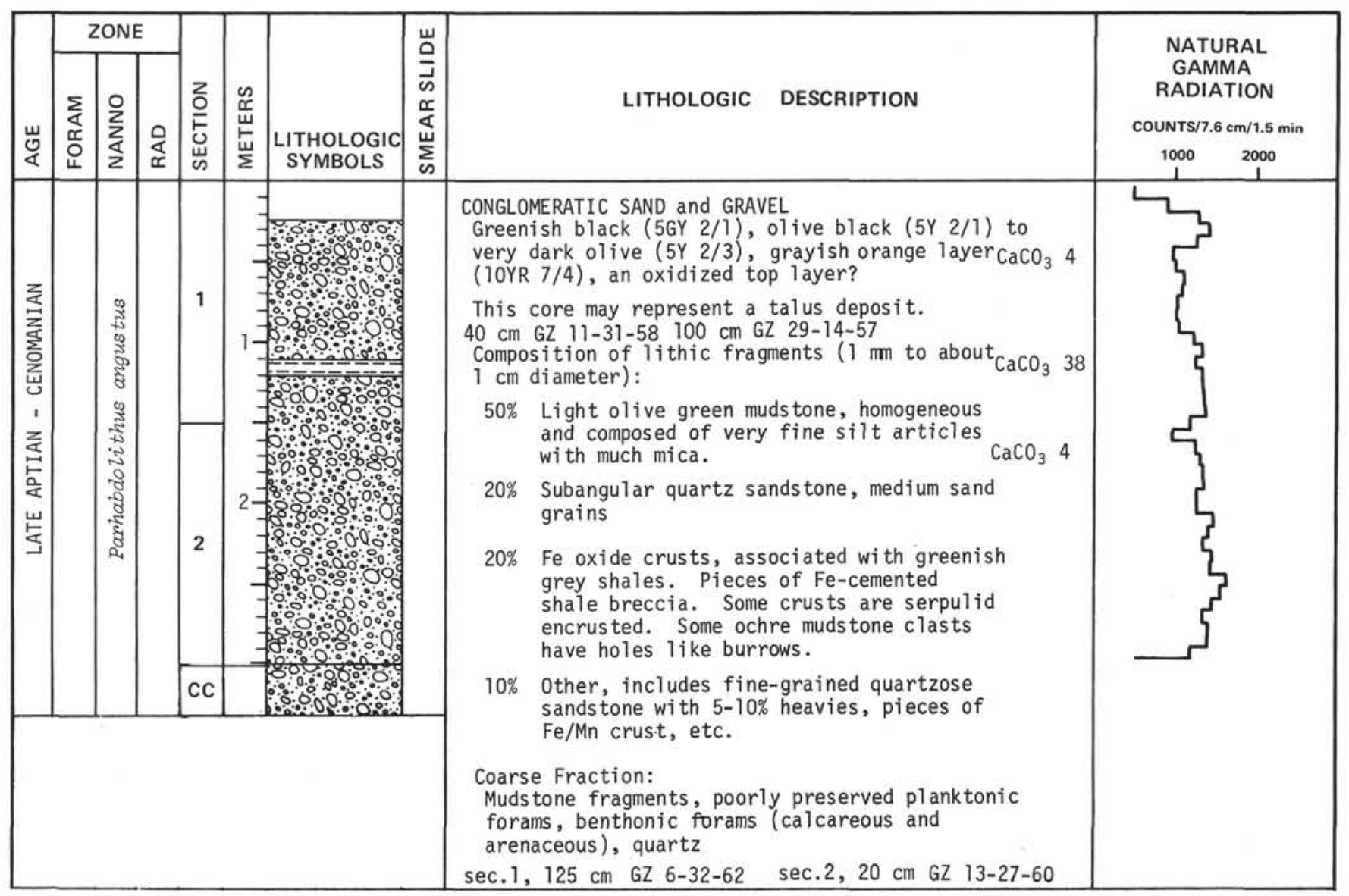




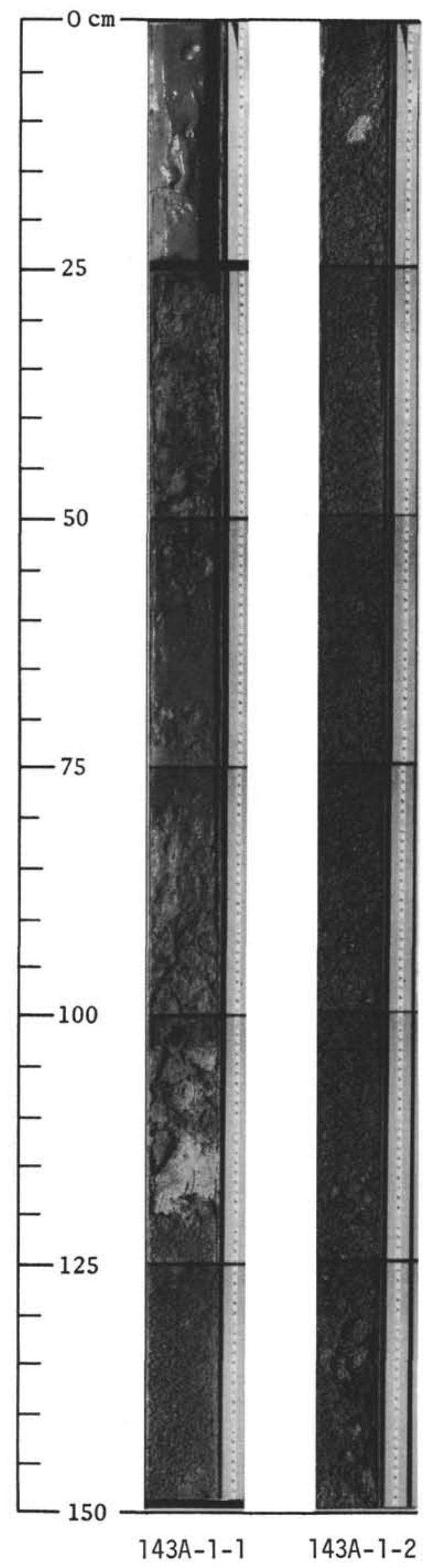


SITE 144

CORE 1

DEPTH (m) 57-65

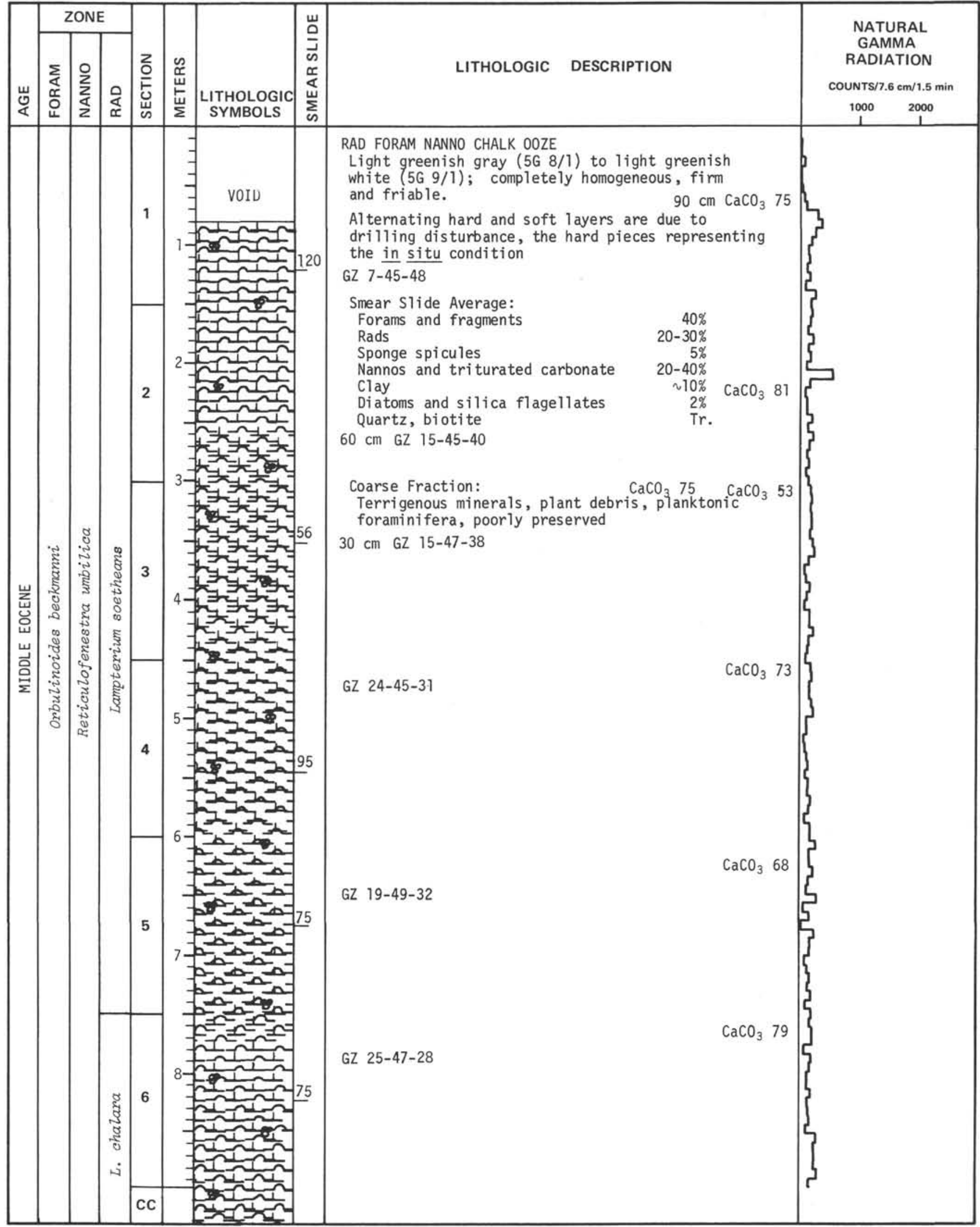




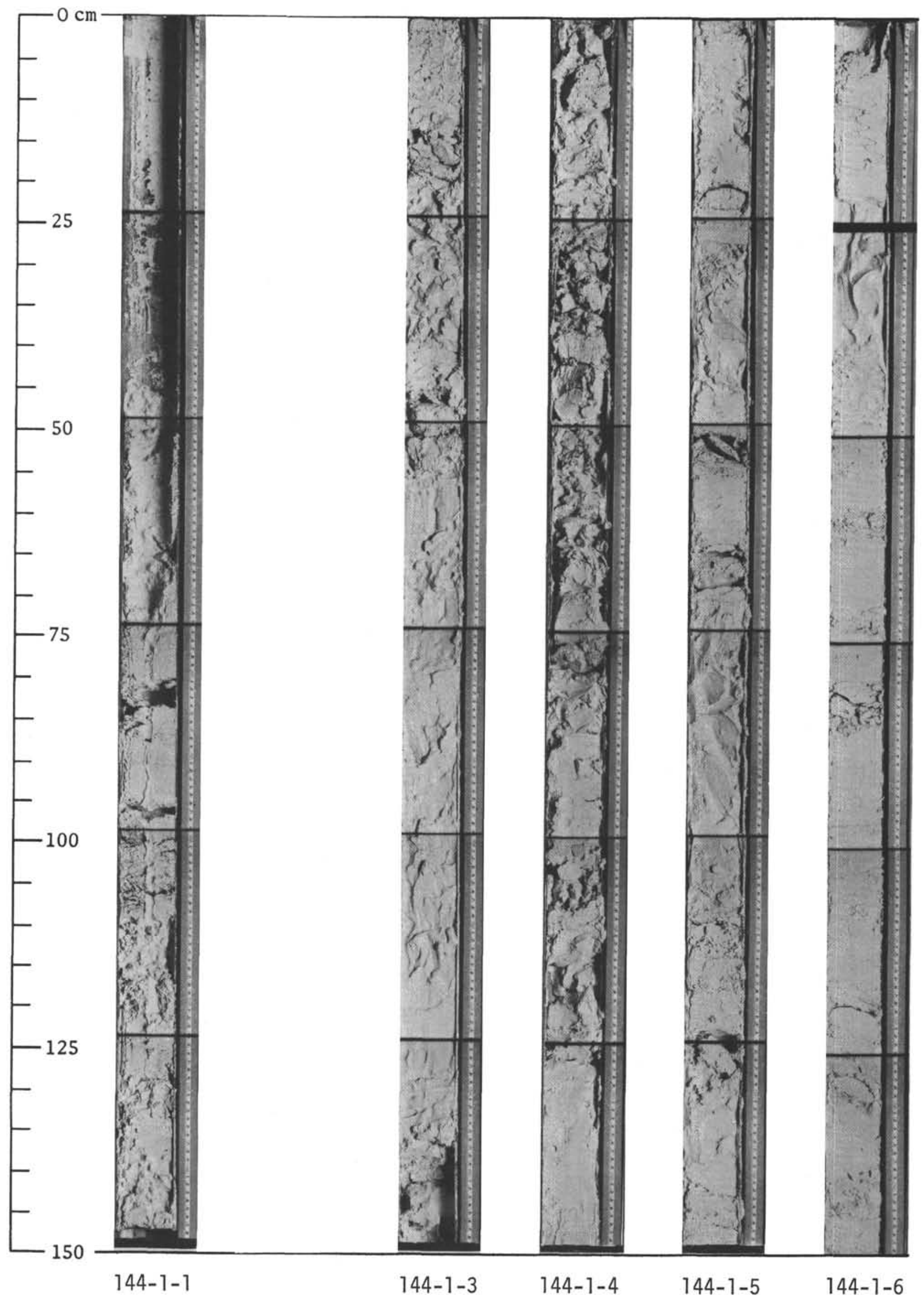


SITE 144

CORE 2

DEPTH (m) 104-112

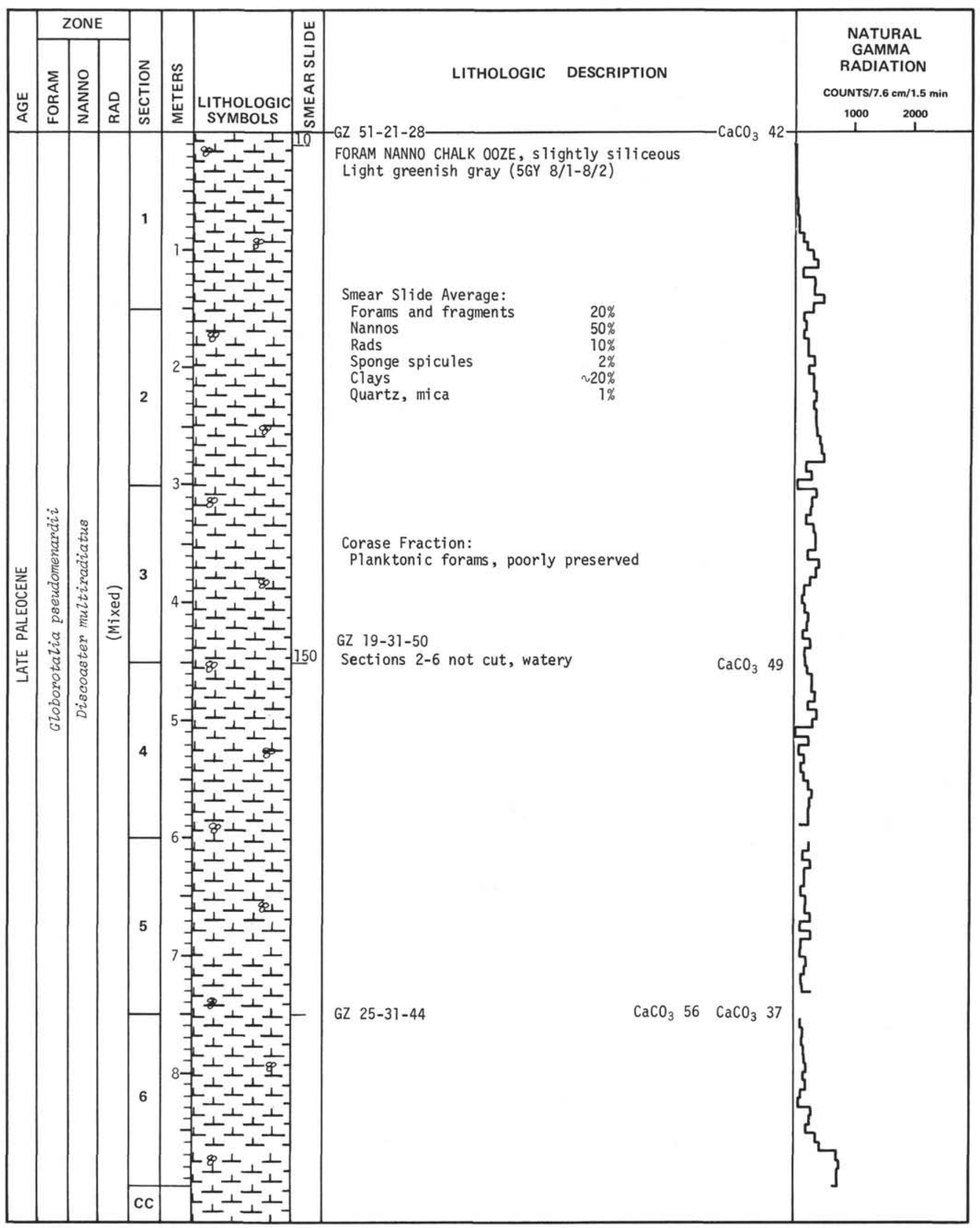




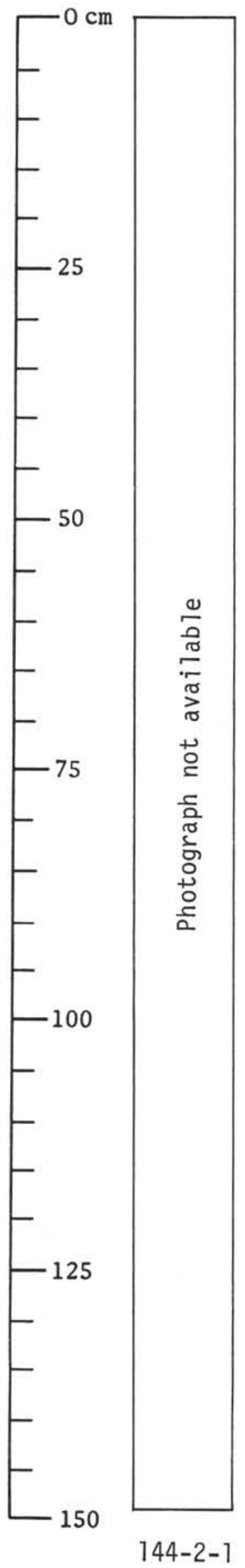


SITE 144

CORE 3

DEPTH (m) 162-166

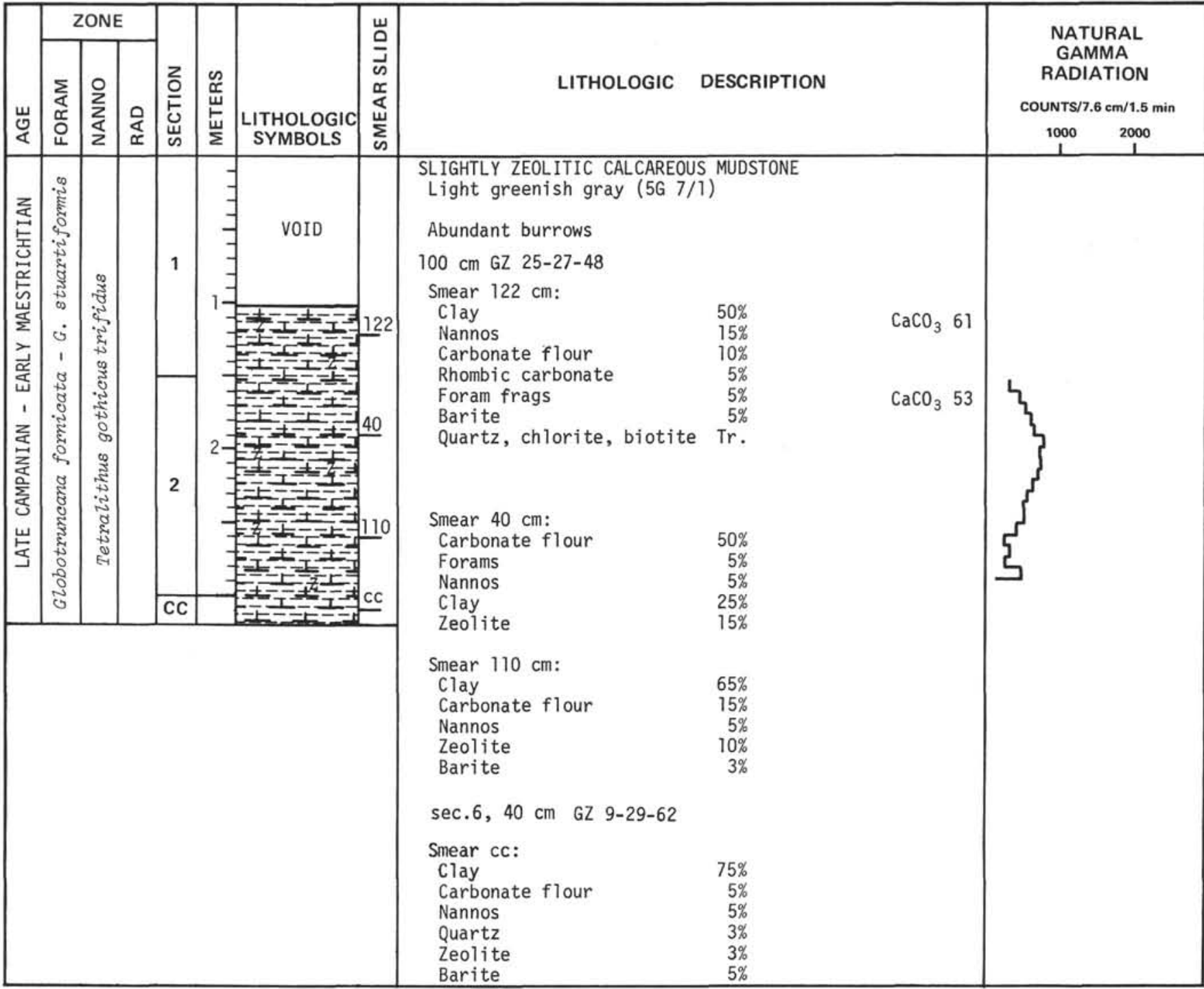




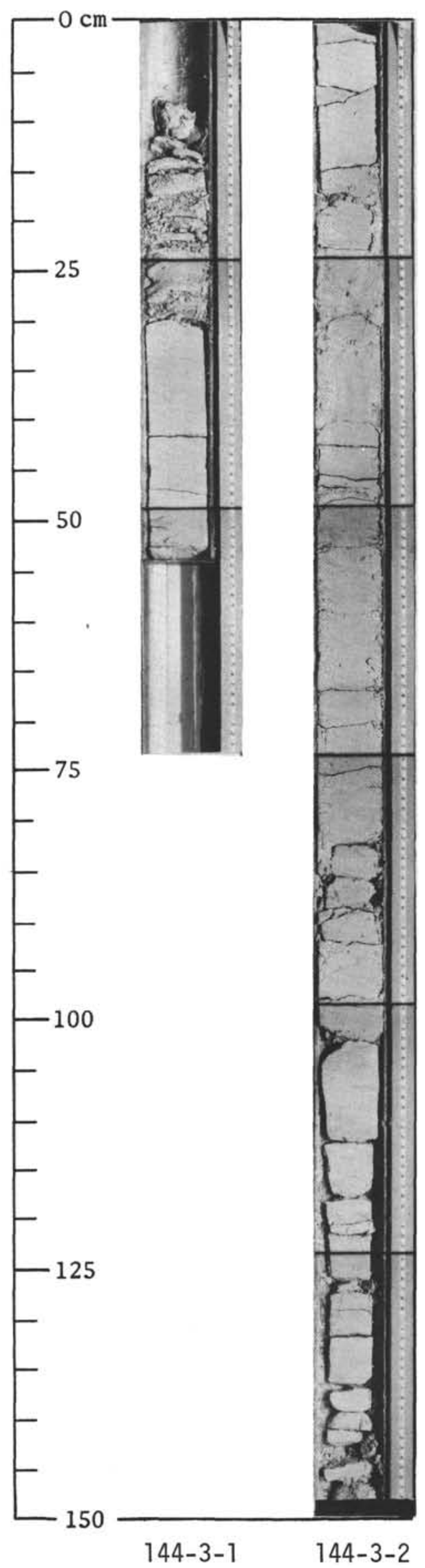


SITE 144

CORE 4

DEPTH (m) 213-219

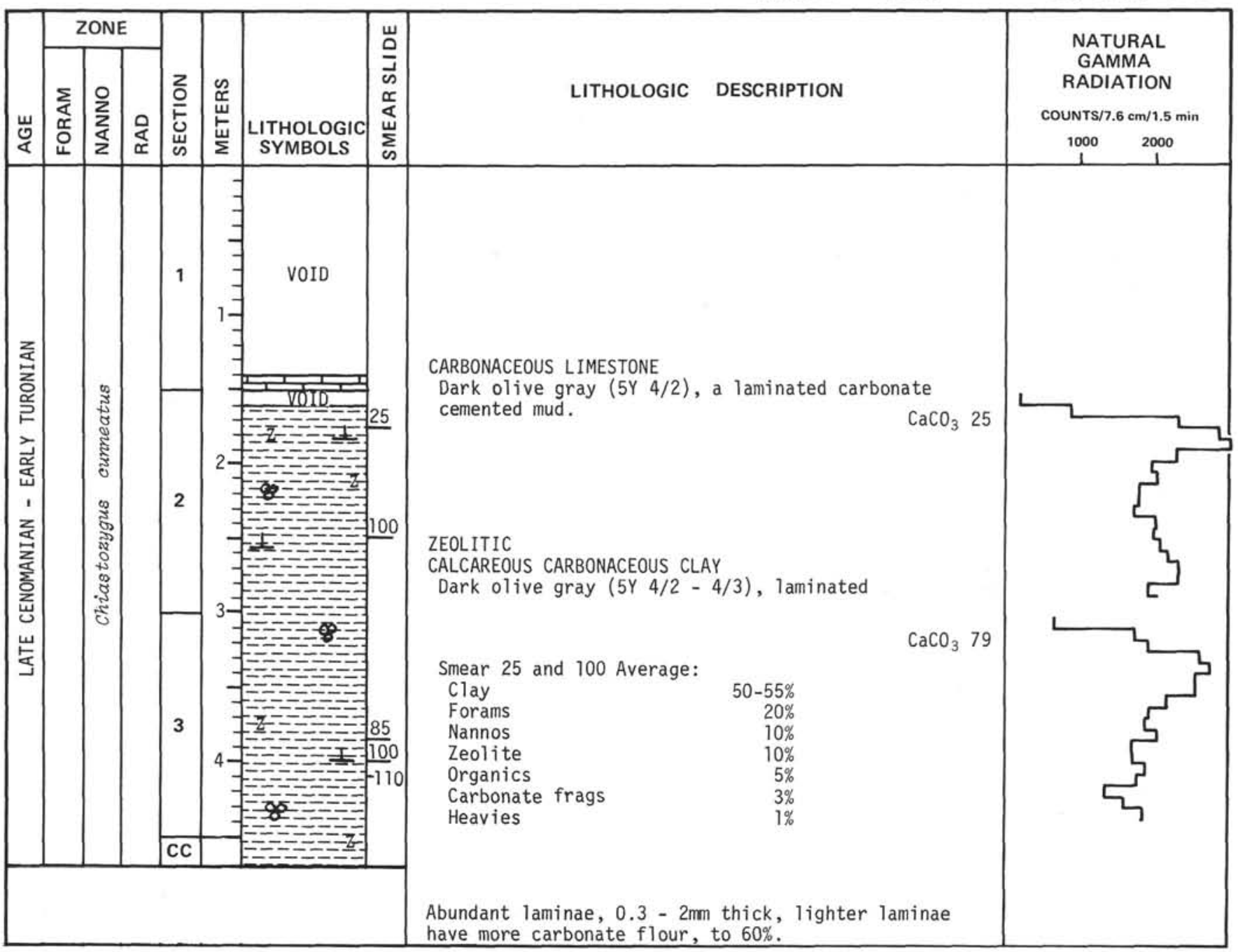




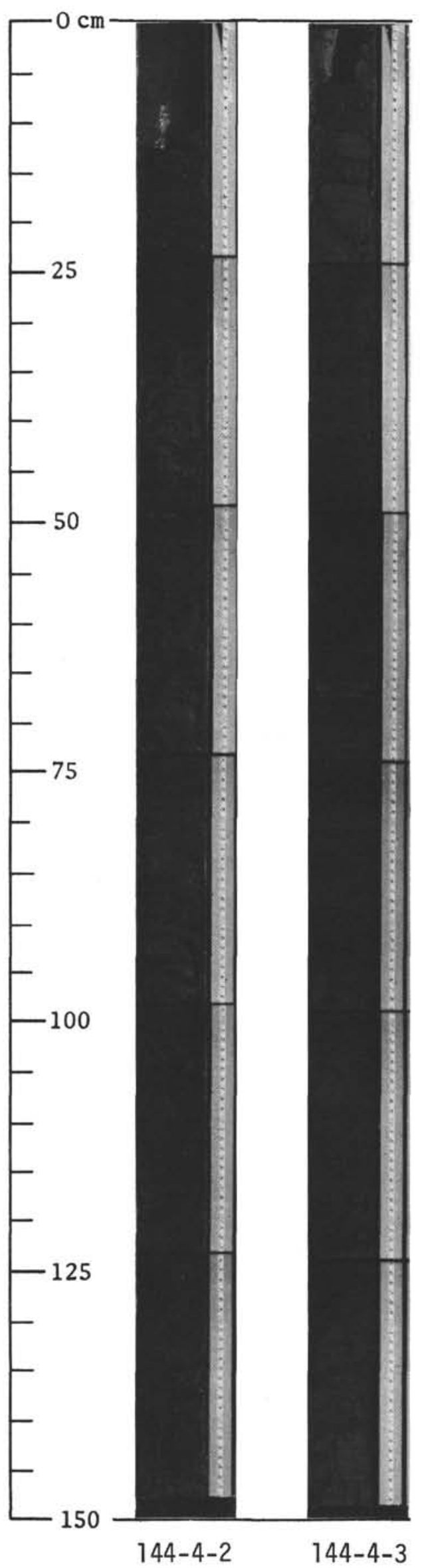


SITE 144

CORE 5

DEPTH (m) 264-270

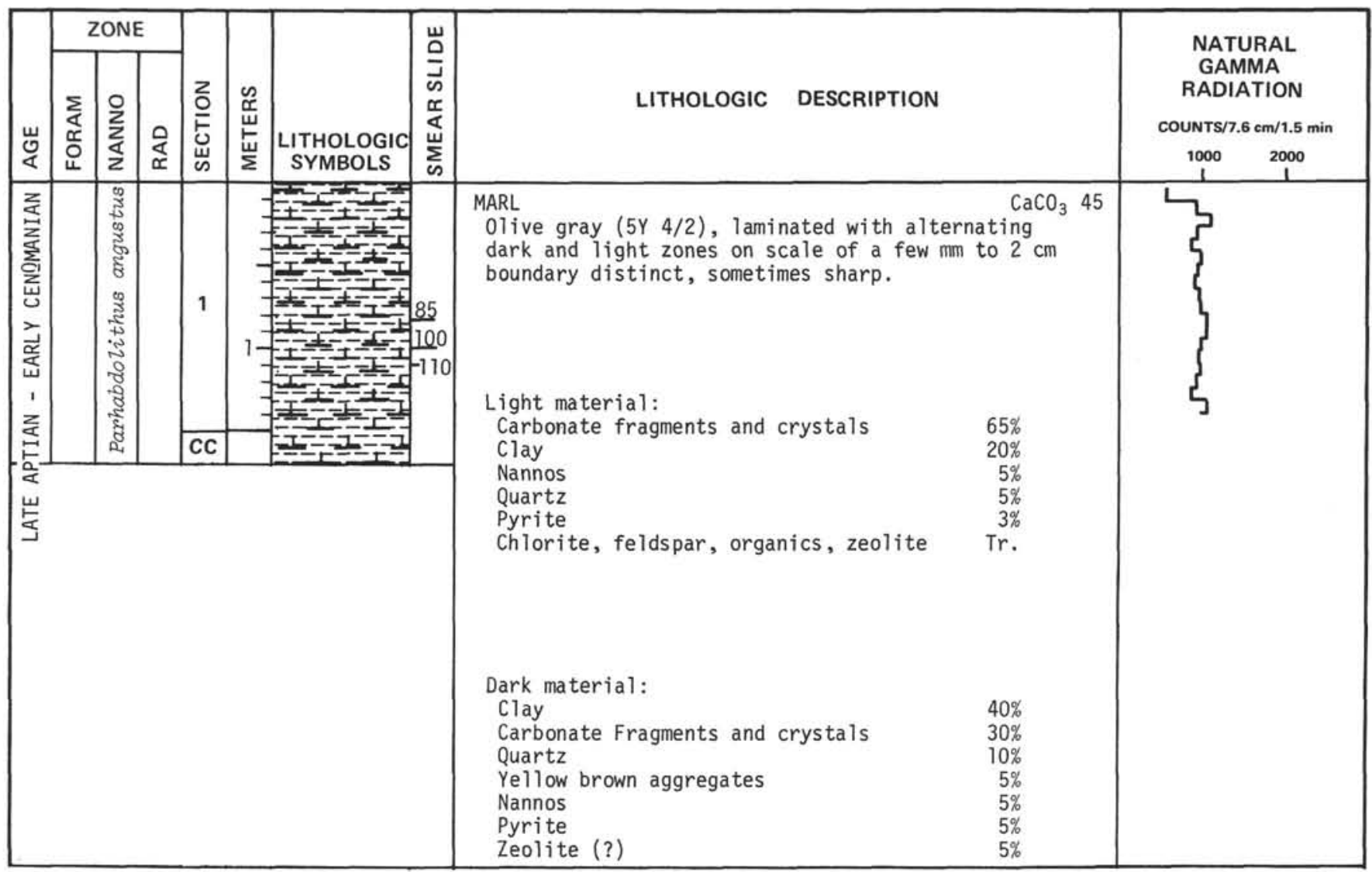




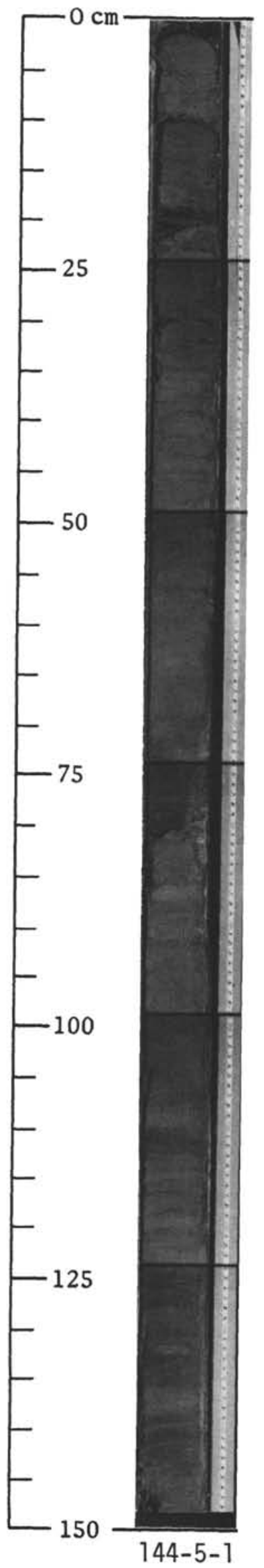




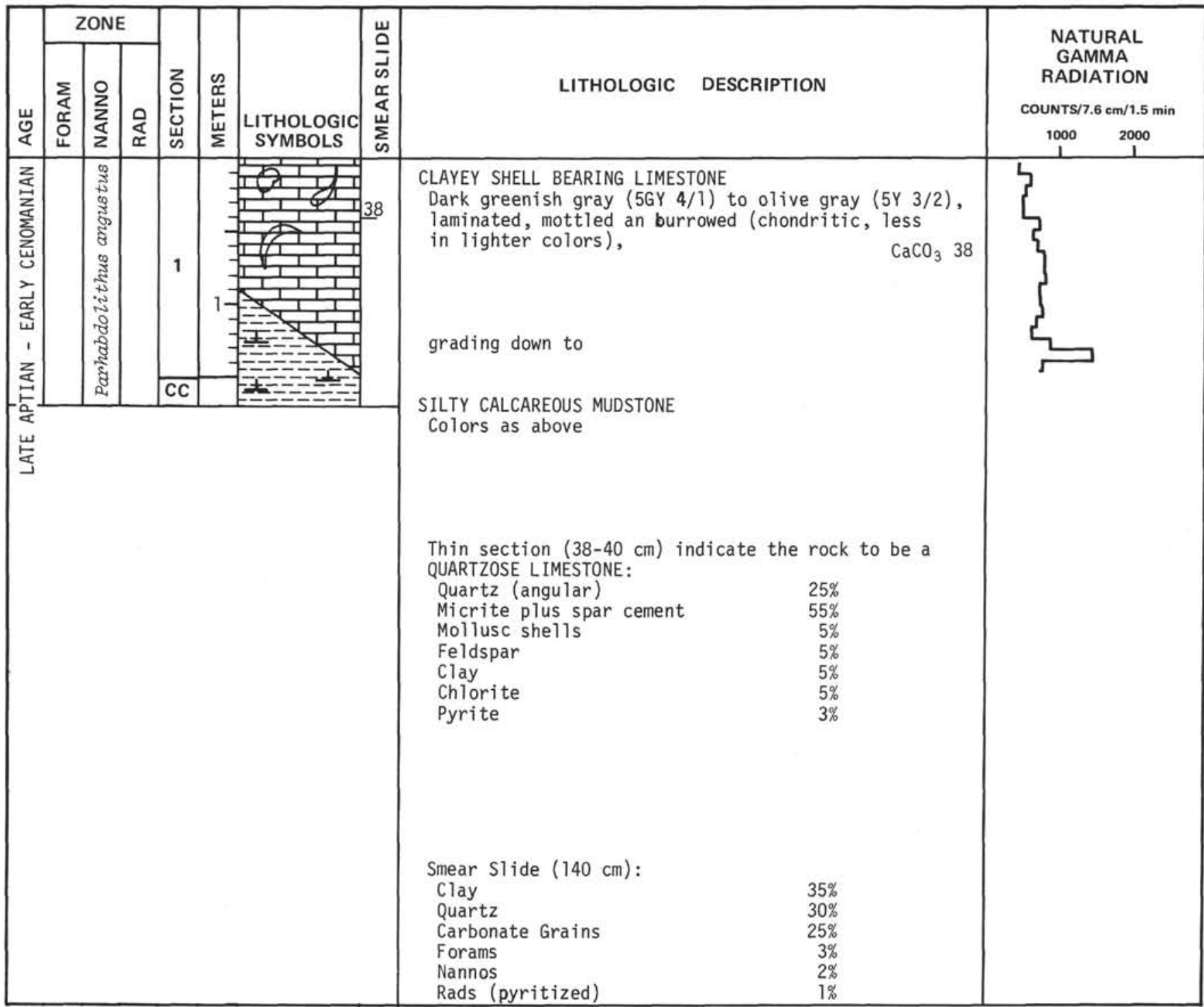




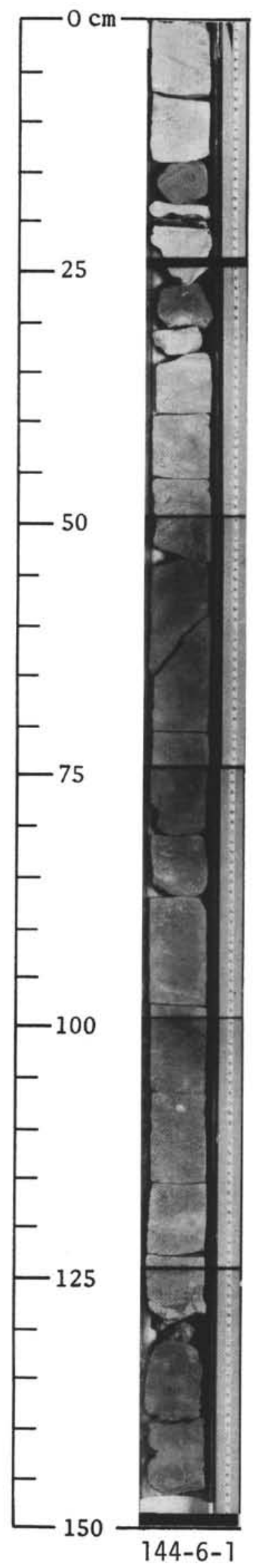


SITE 144

CORE 7

DEPTH (m) 298-300

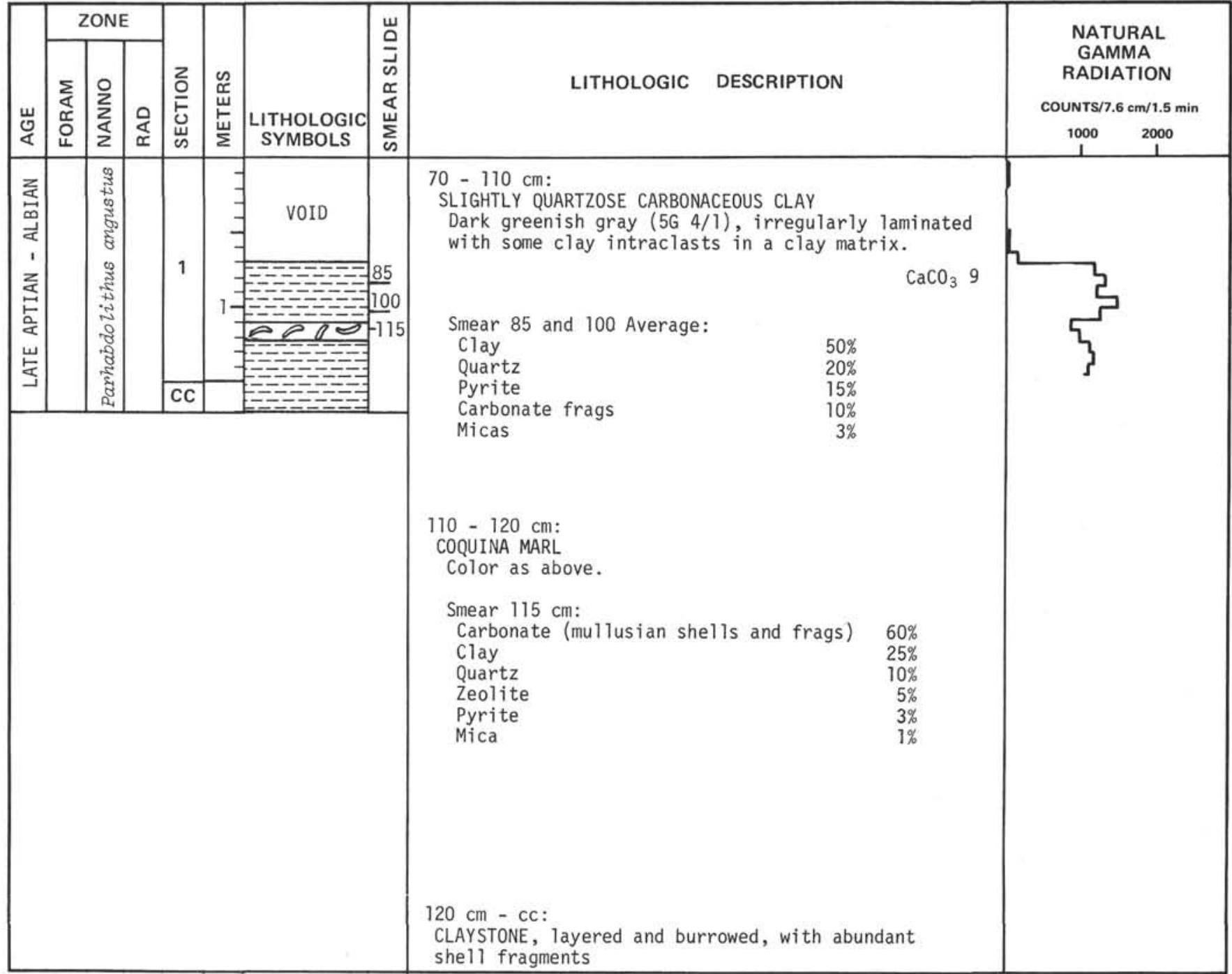




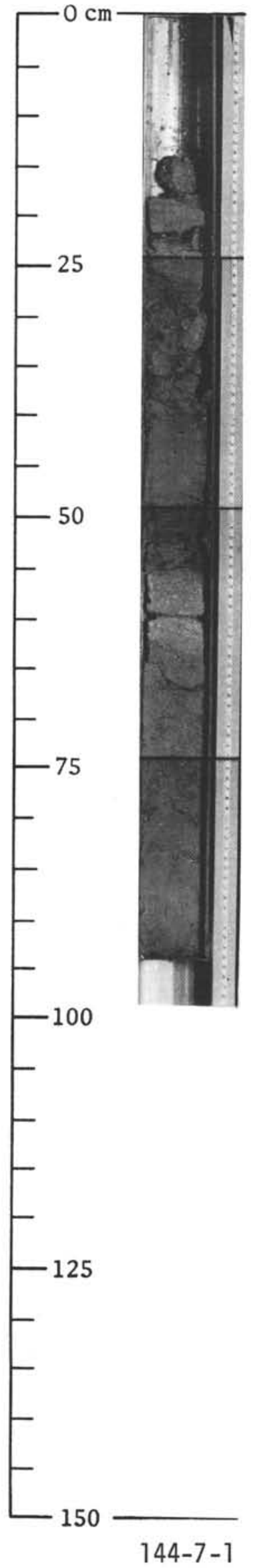


SITE 144

CORE 8

DEPTH (m) 324-327

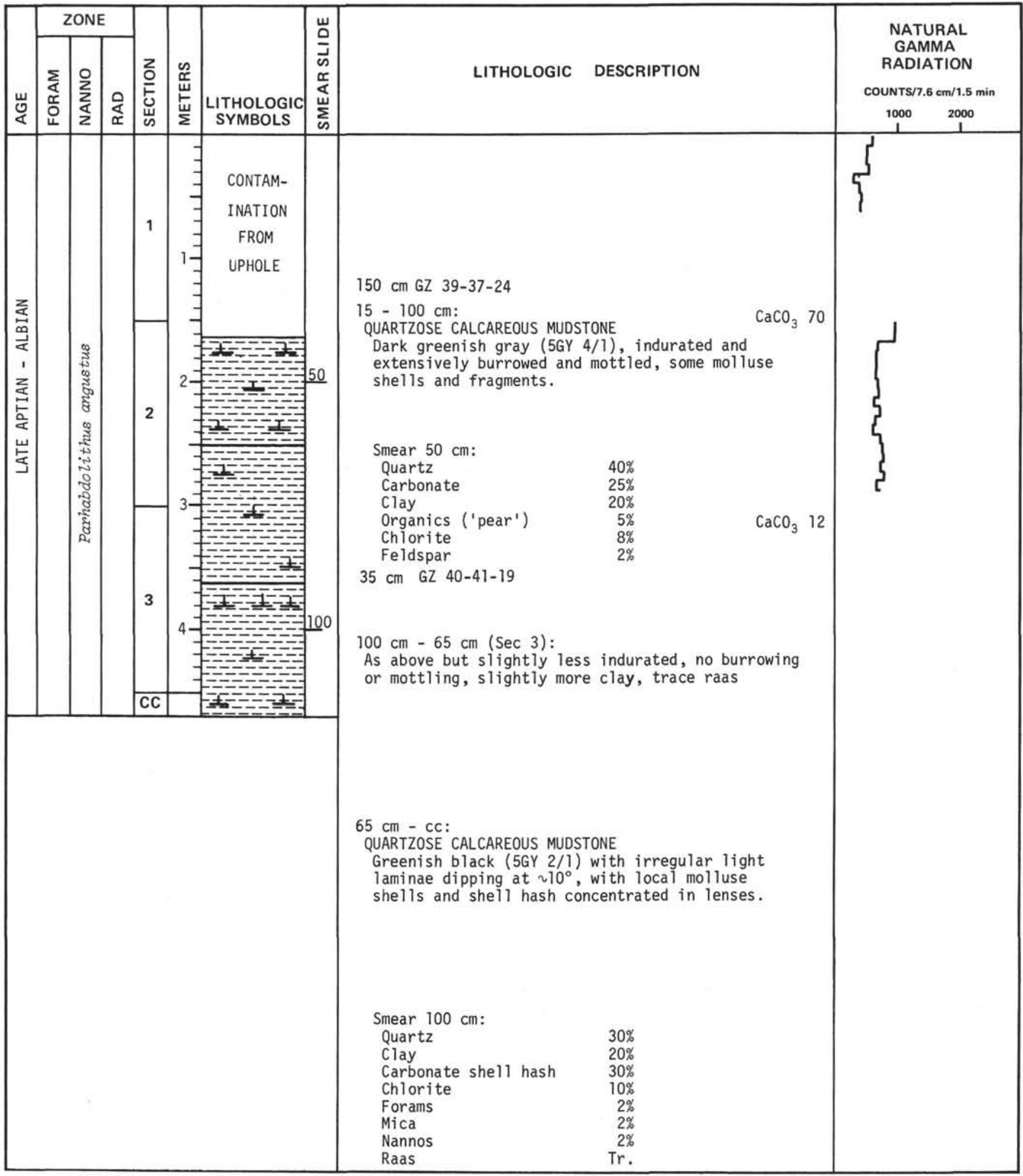




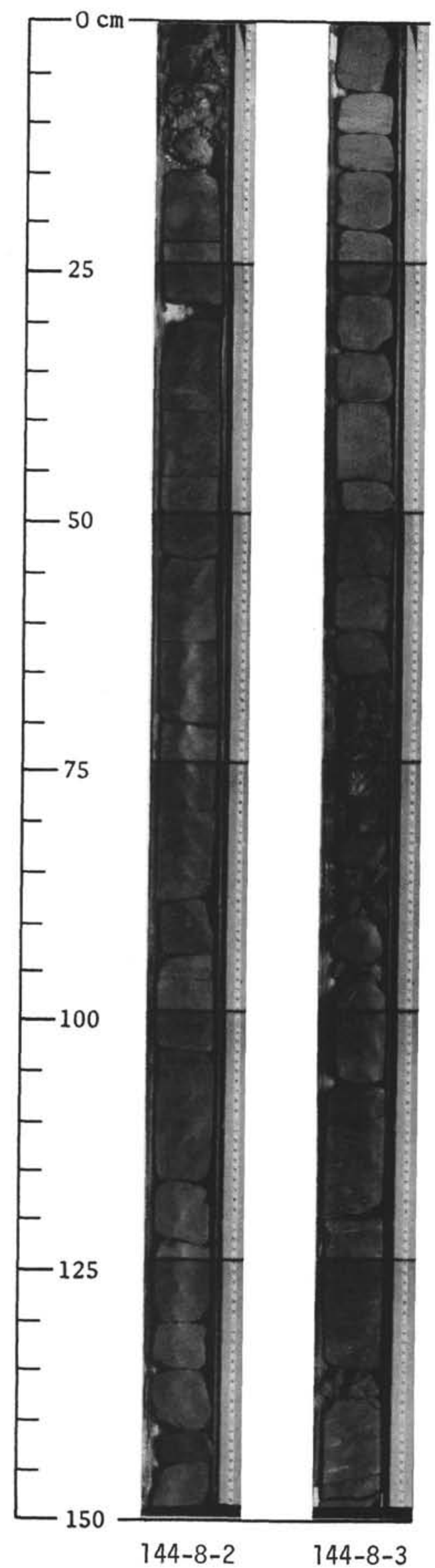


SITE 144A

CORE I

DEPTH (m) 20-29

\begin{tabular}{|c|c|c|c|c|c|c|c|c|c|c|}
\hline 岁 & 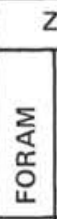 & $\begin{array}{l}0 \\
2 \\
2 \\
\vdots \\
2\end{array}$ & 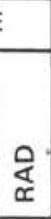 & 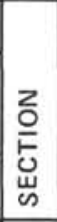 & $\begin{array}{l}\mathscr{w} \\
\stackrel{\sim}{w} \\
\stackrel{w}{w} \\
\Sigma\end{array}$ & $\begin{array}{l}\text { LITHOLOGIC } \\
\text { SYMBOLS }\end{array}$ & 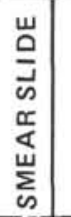 & LITHOLOGIC DESCRIPTION & \multicolumn{2}{|c|}{$\begin{array}{c}\text { NATURAL } \\
\text { GAMMA } \\
\text { RADIATION } \\
\text { CoUNTS } / 7.6 \mathrm{~cm} / 1.5 \mathrm{~min}\end{array}$} \\
\hline 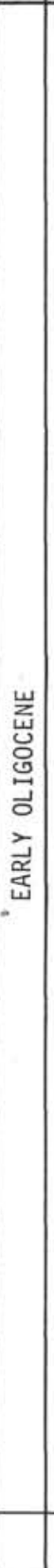 & 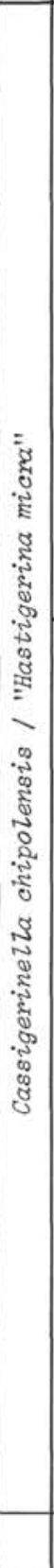 & 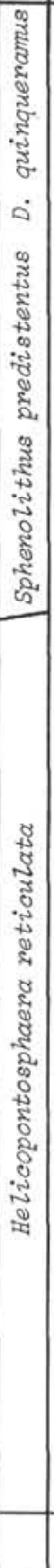 & 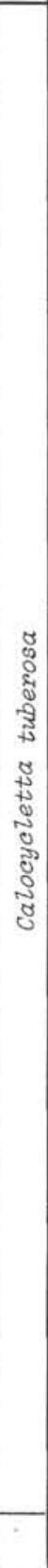 & $\mathrm{cc}$ & $6=$ & $\frac{1-1 \perp 1}{1 \perp \perp \perp}$ & $\begin{array}{l}120 \\
25 \\
-\end{array}$ & 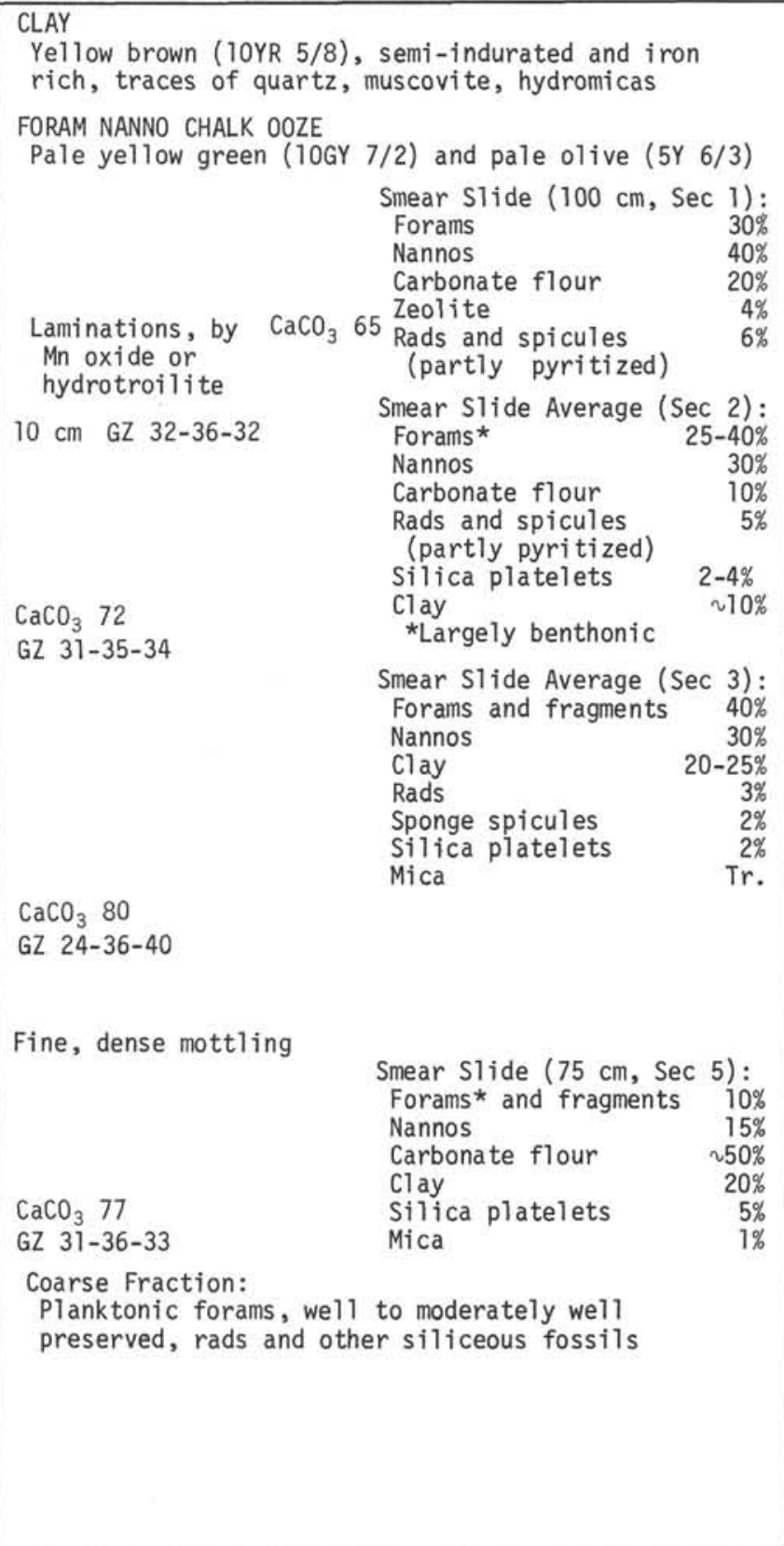 & 3 & \\
\hline
\end{tabular}




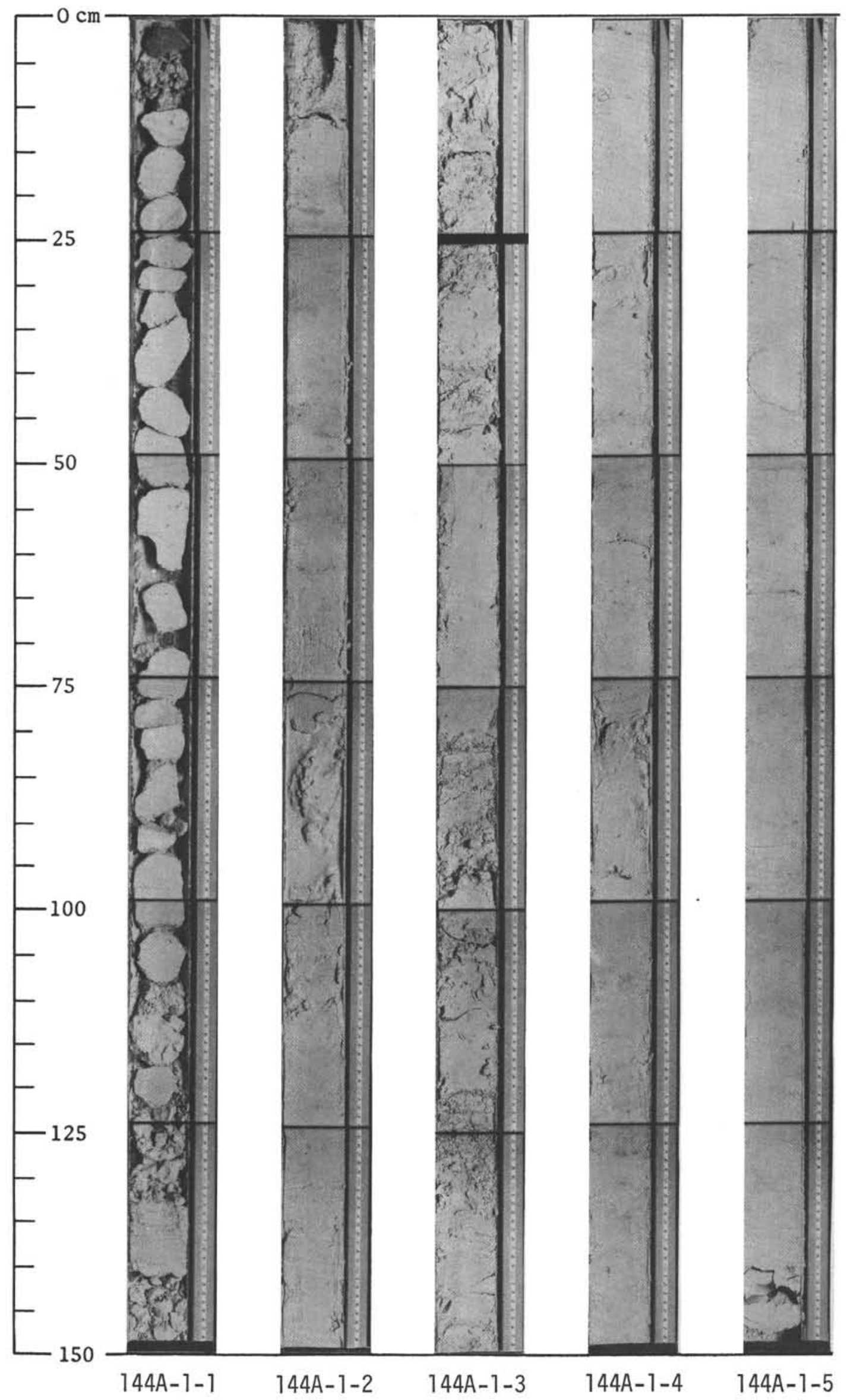


SITE 144A

CORE 2

DEPTH (m) 38-47

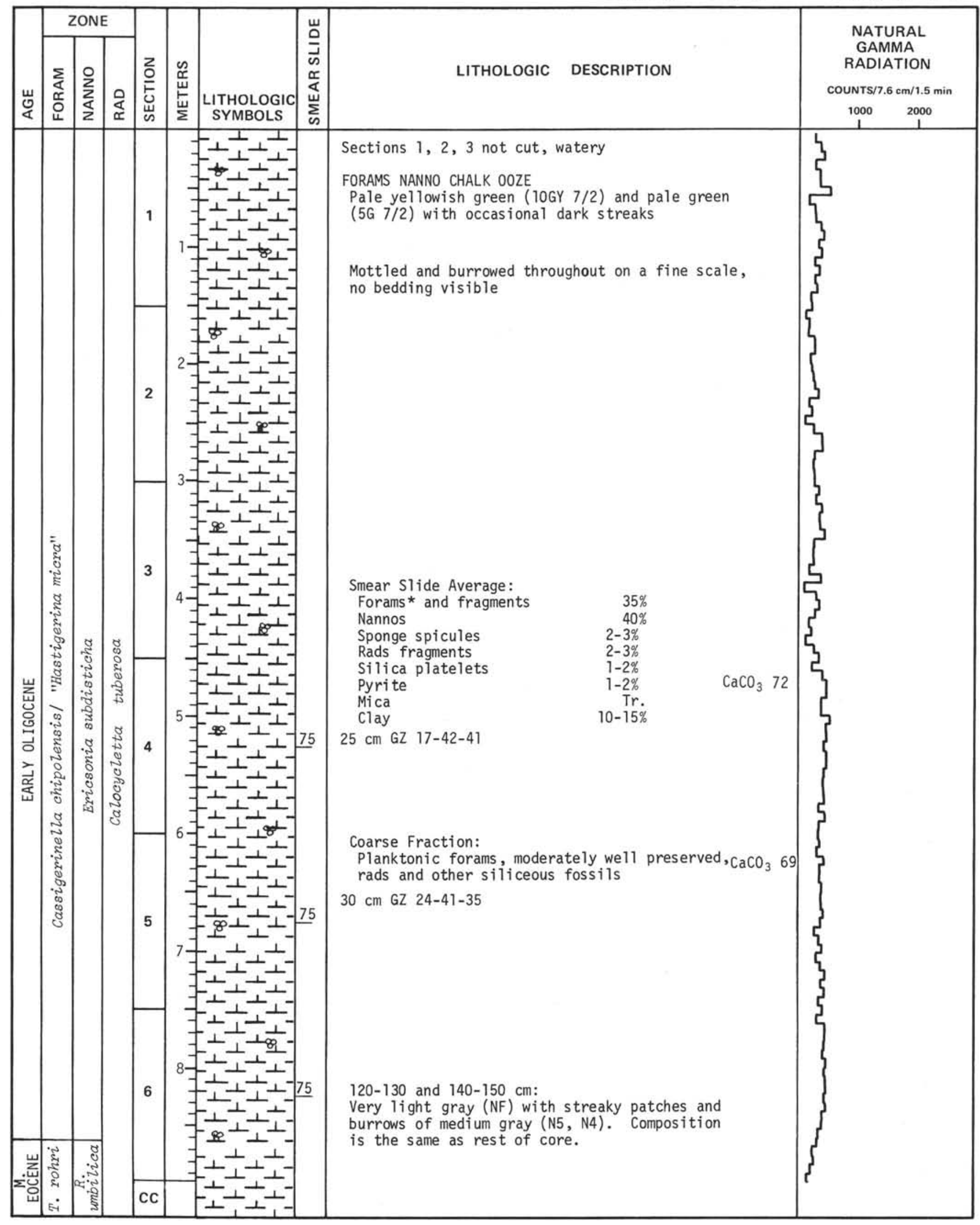




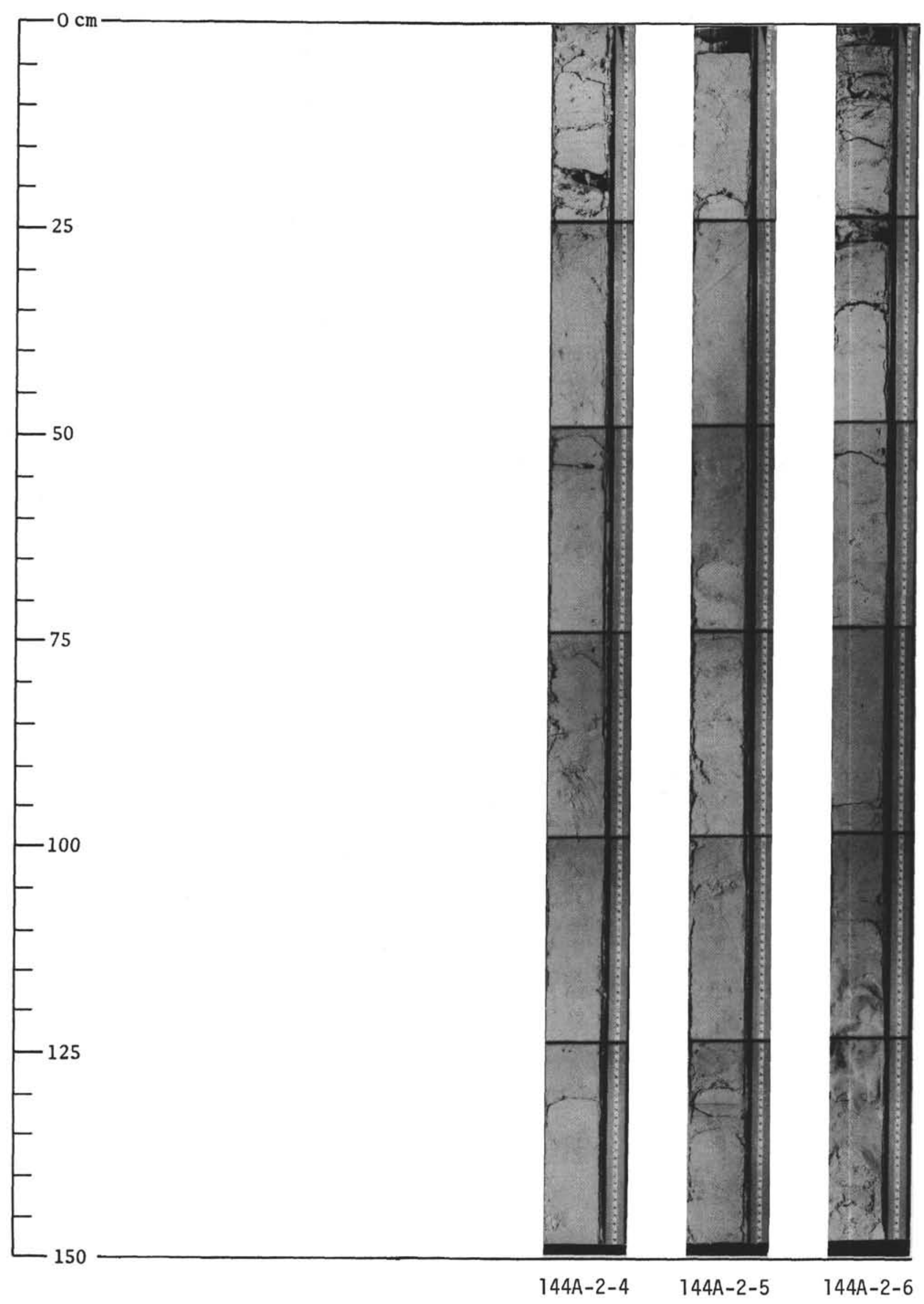


SITE 144A

CORE 3

DEPTH (m) 140-149

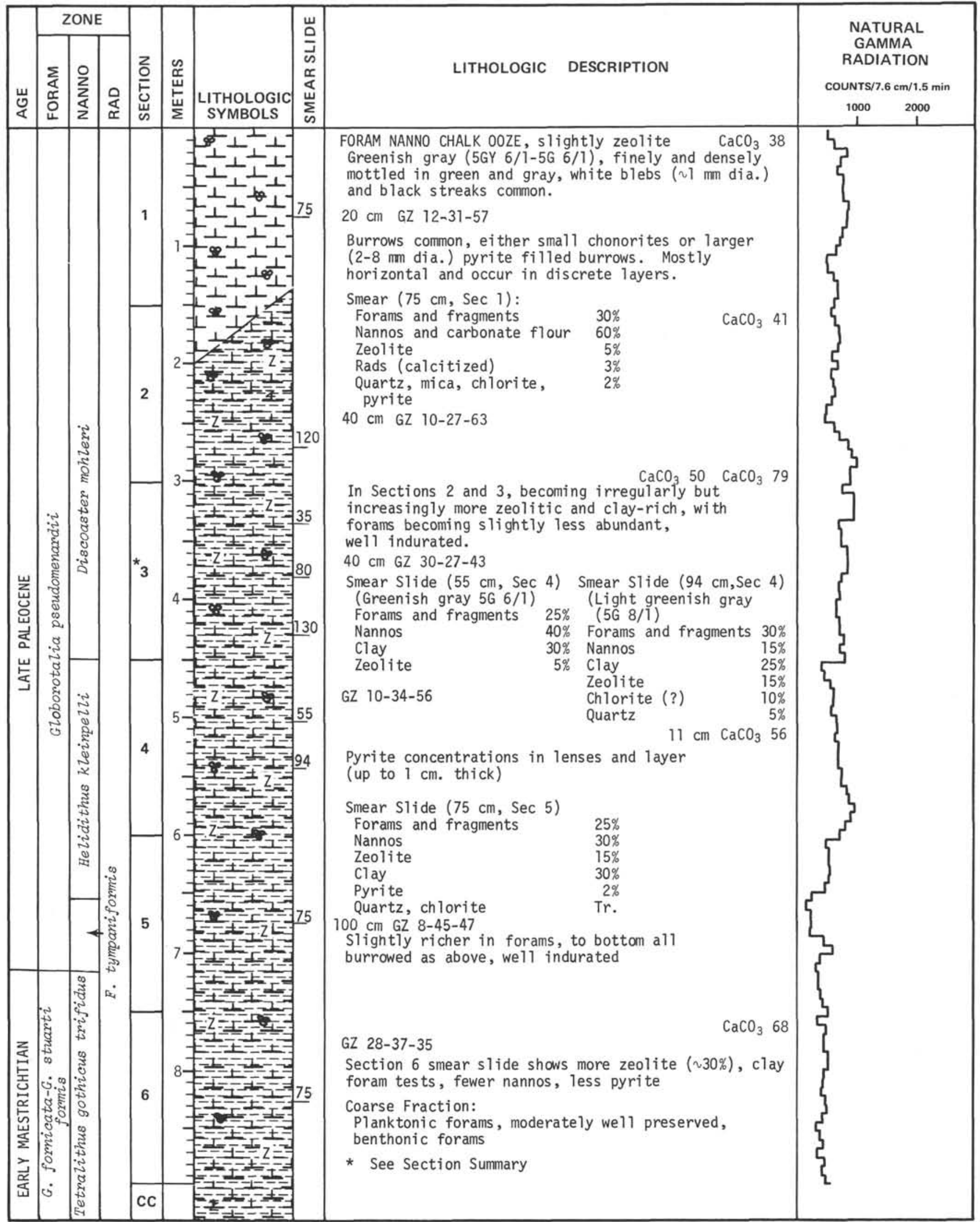




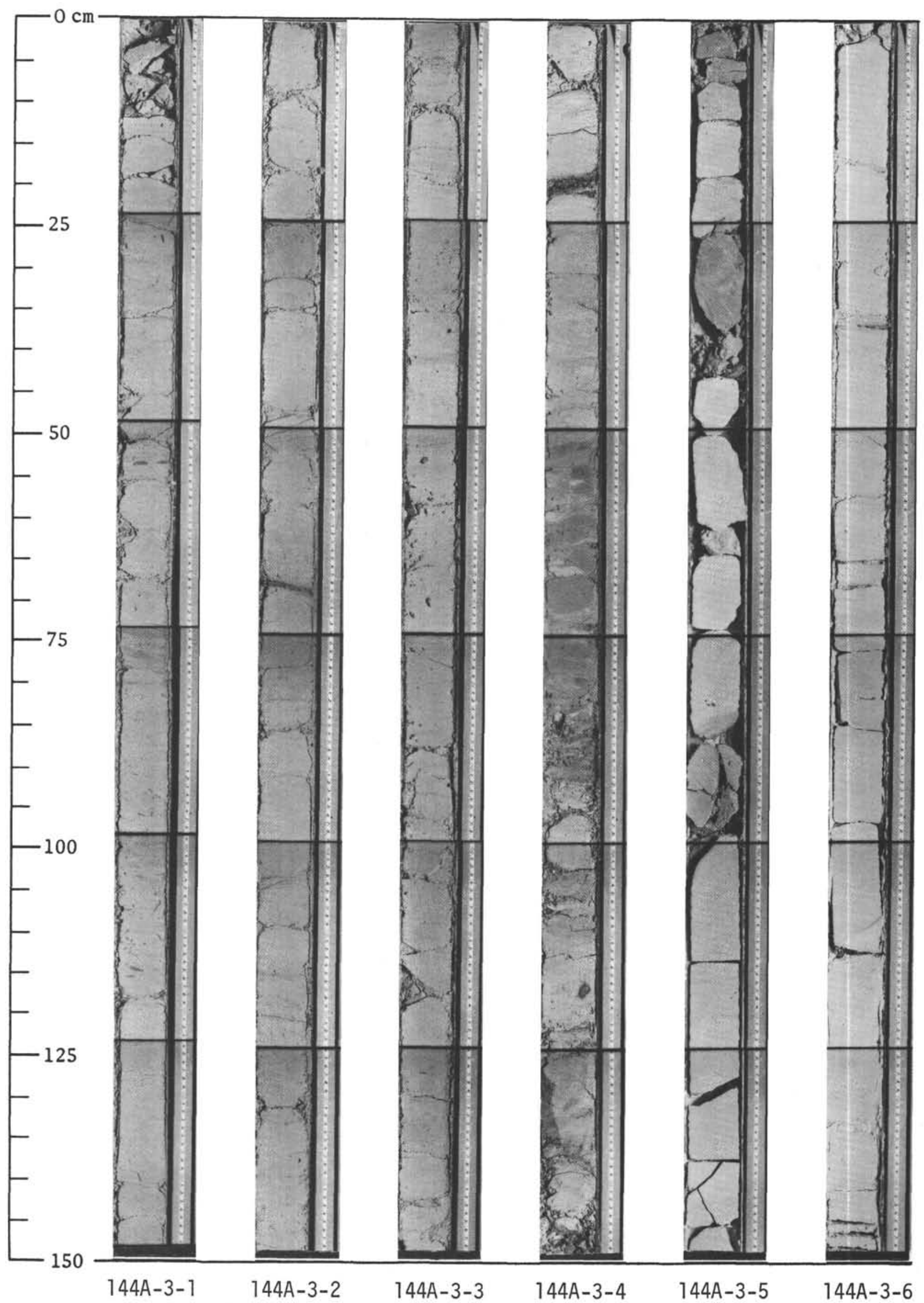


SITE 144A

CORE 4

DEPTH (m) 171-180

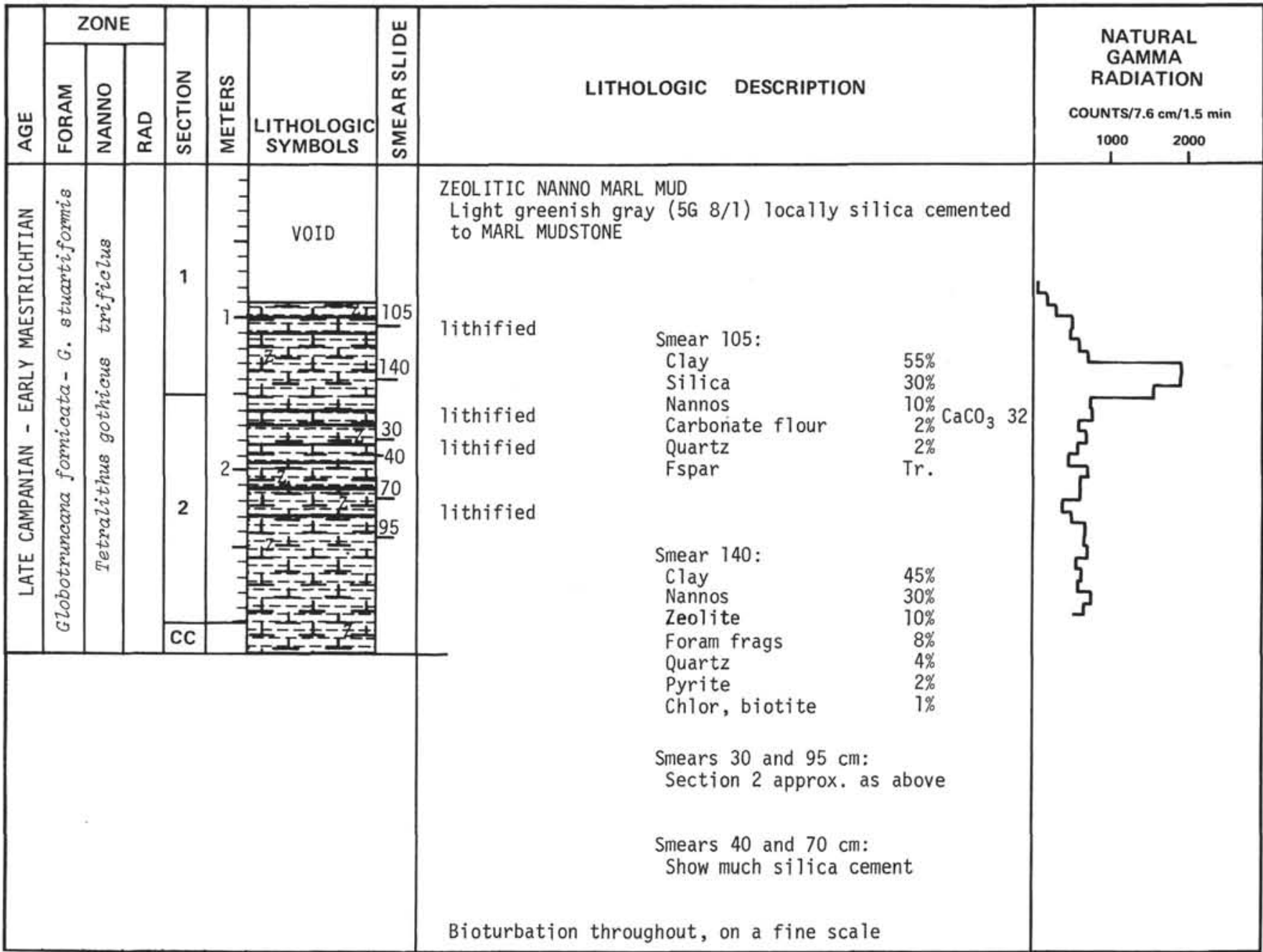




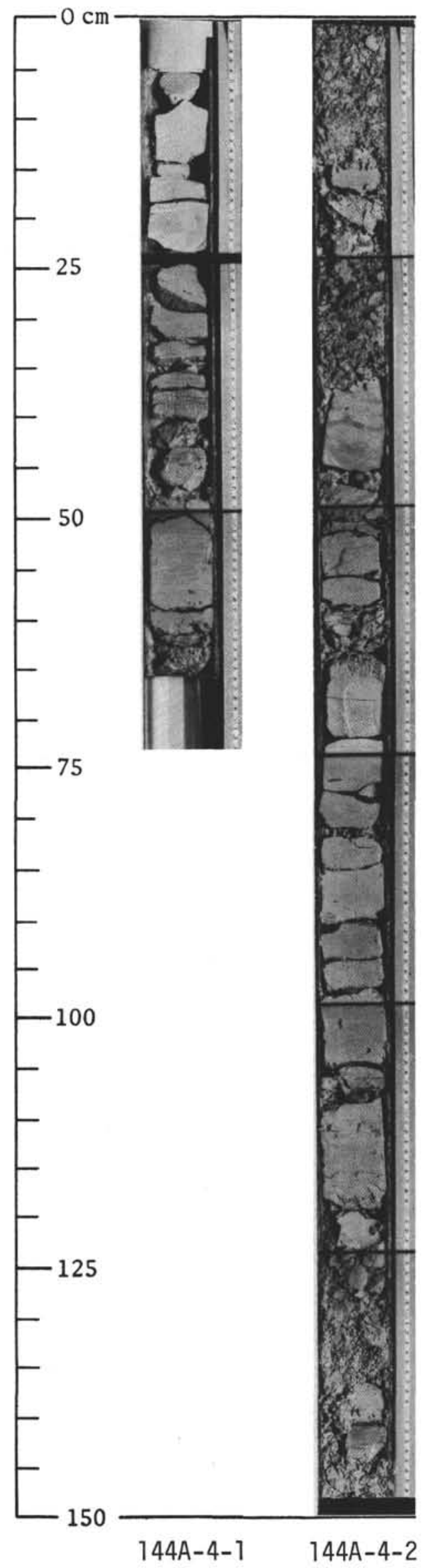


SITE 144A

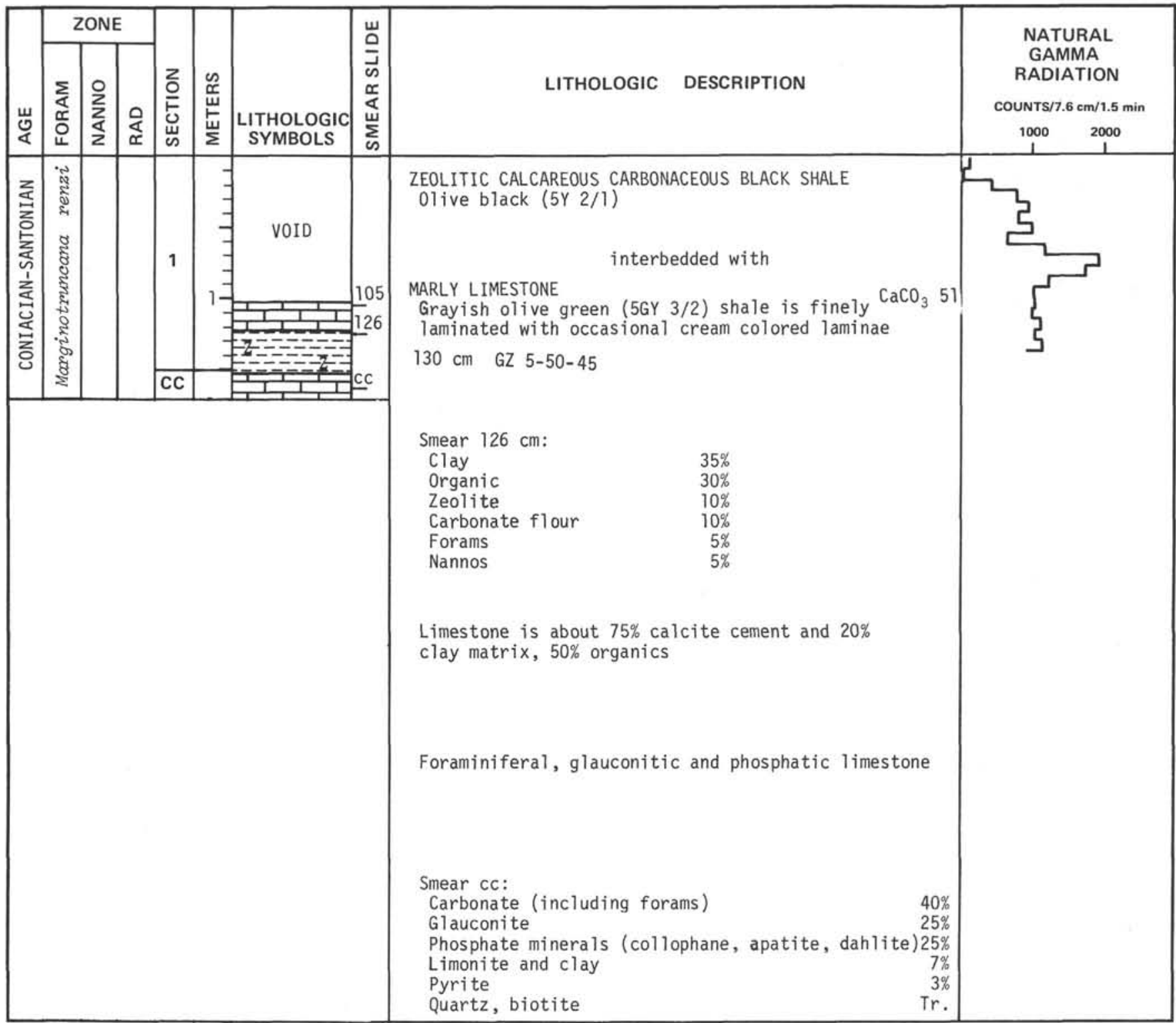

SITE 144A

CORE 6

DEPTH (m) 189-197

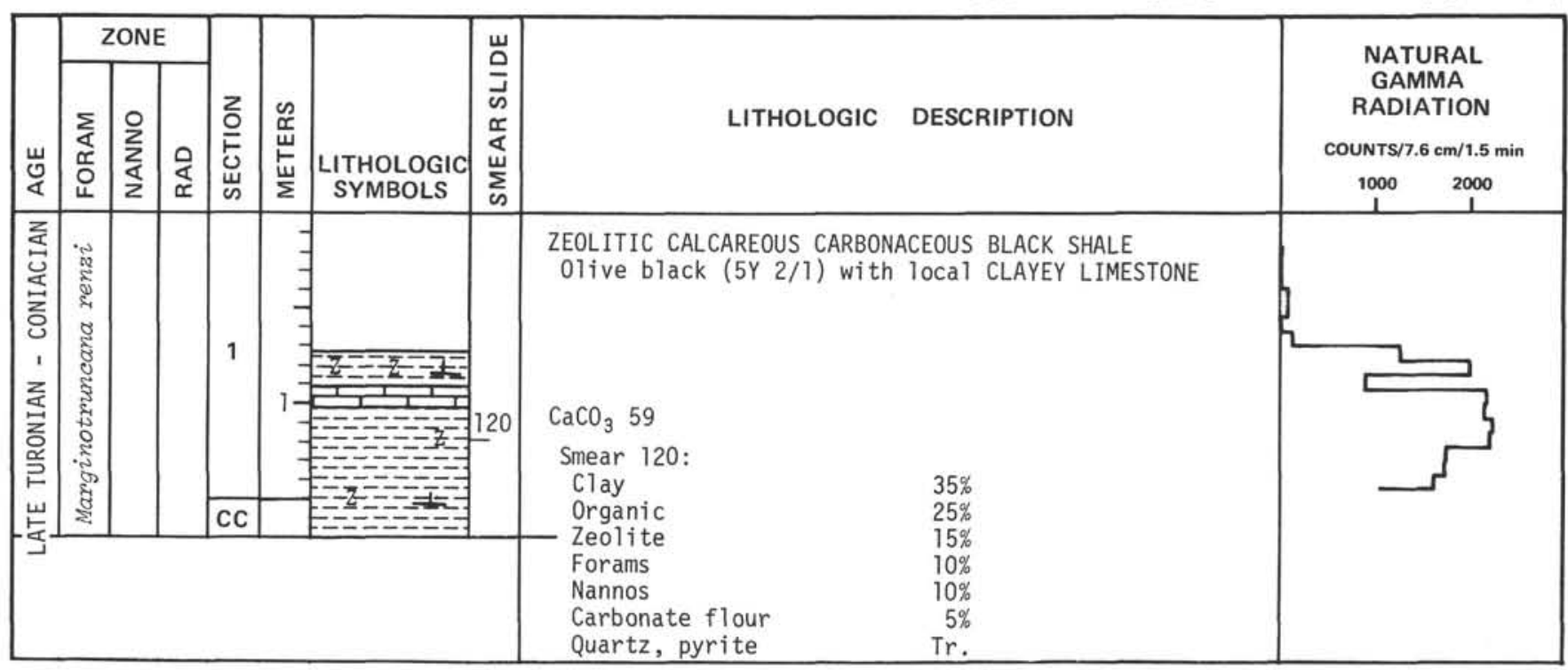




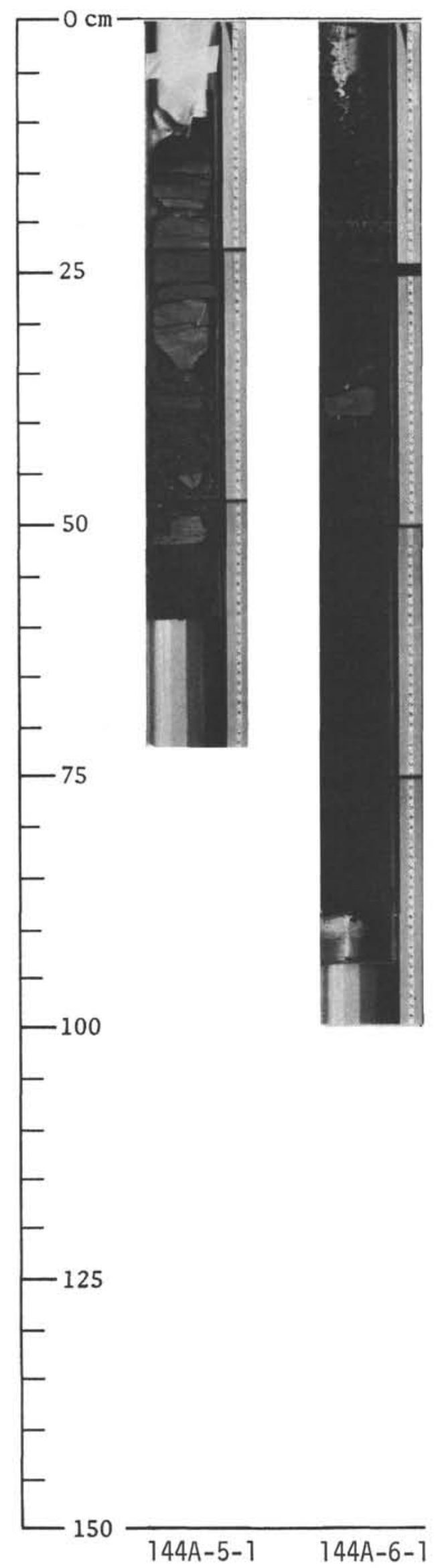




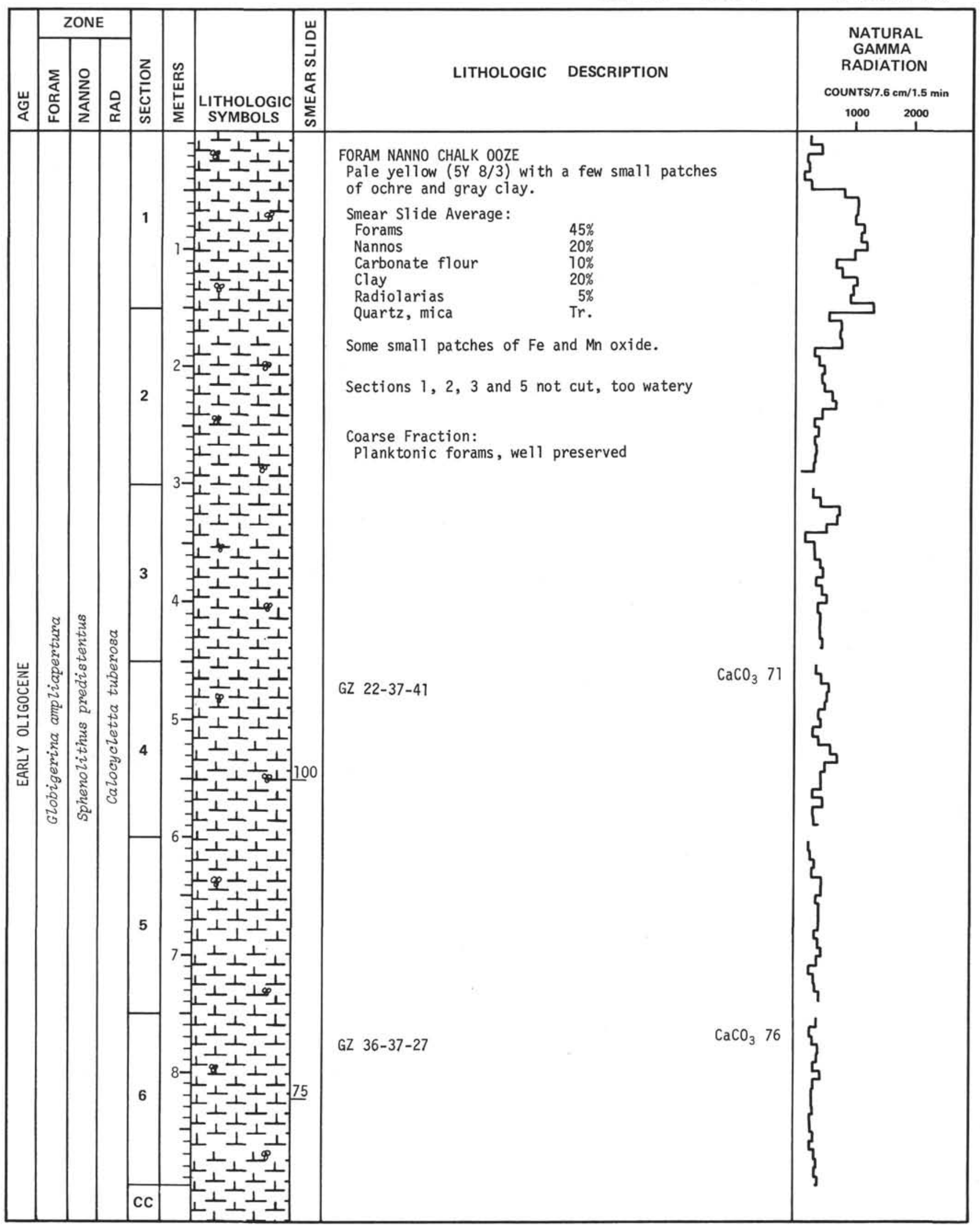


SITES 143 AND 144

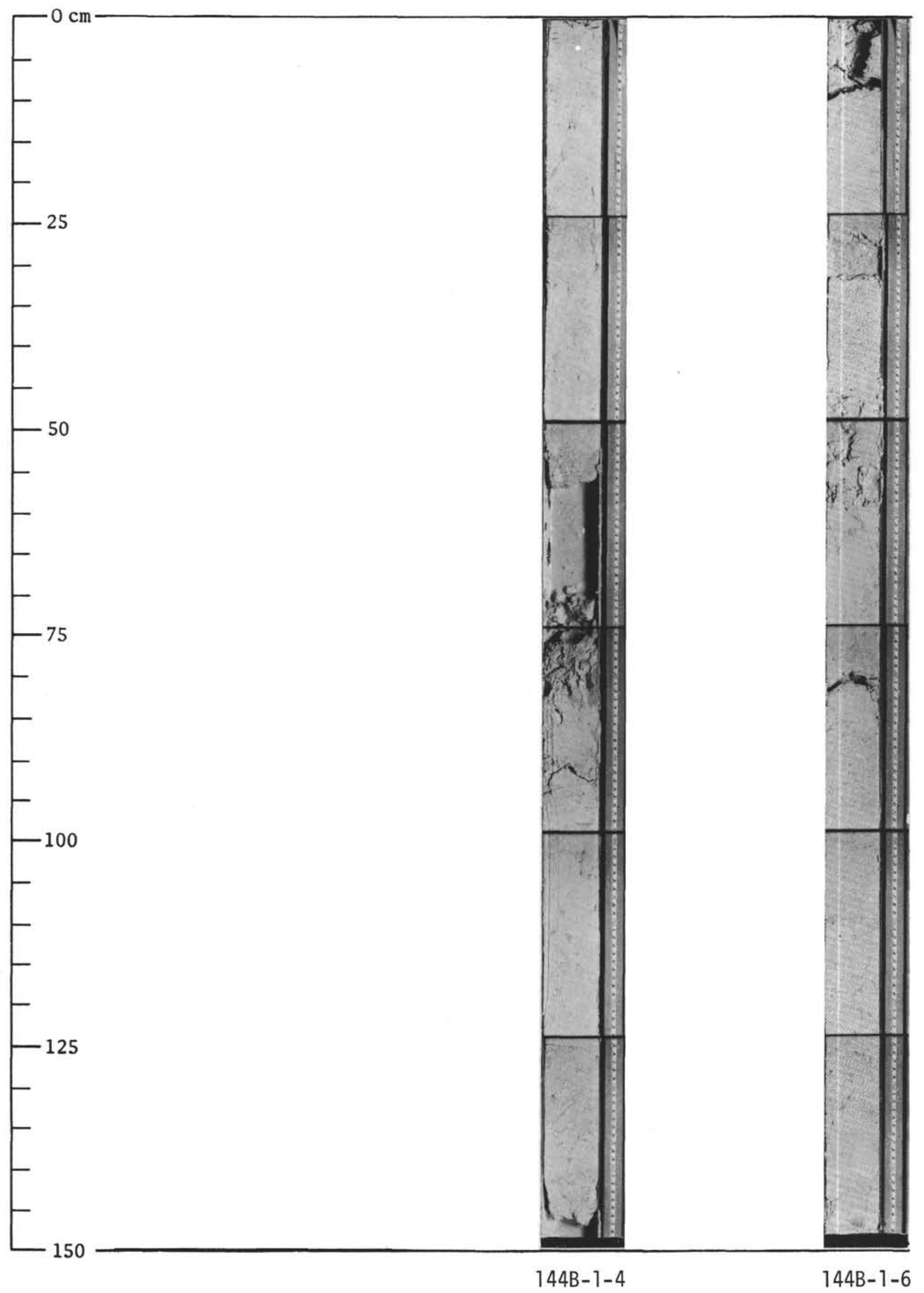




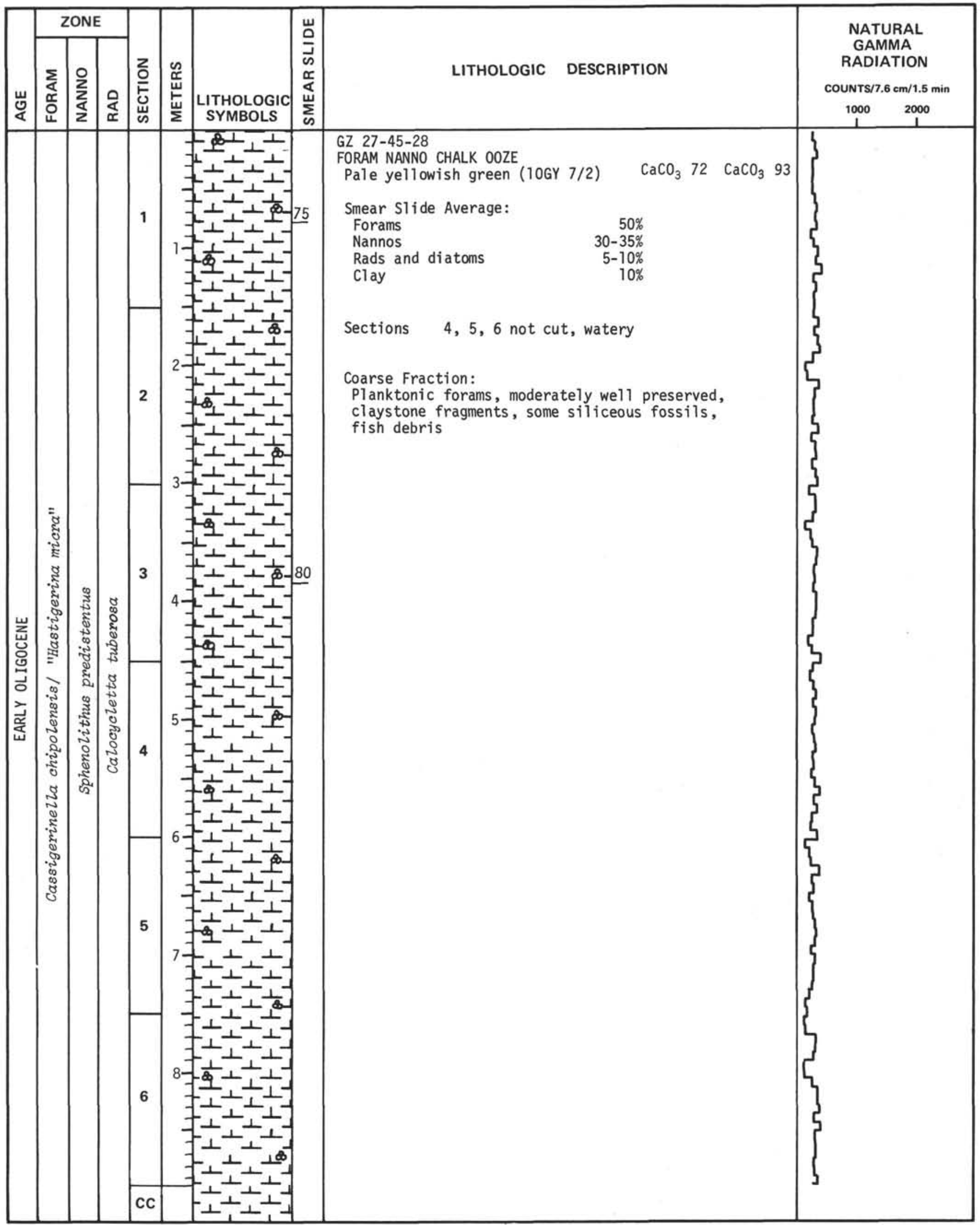




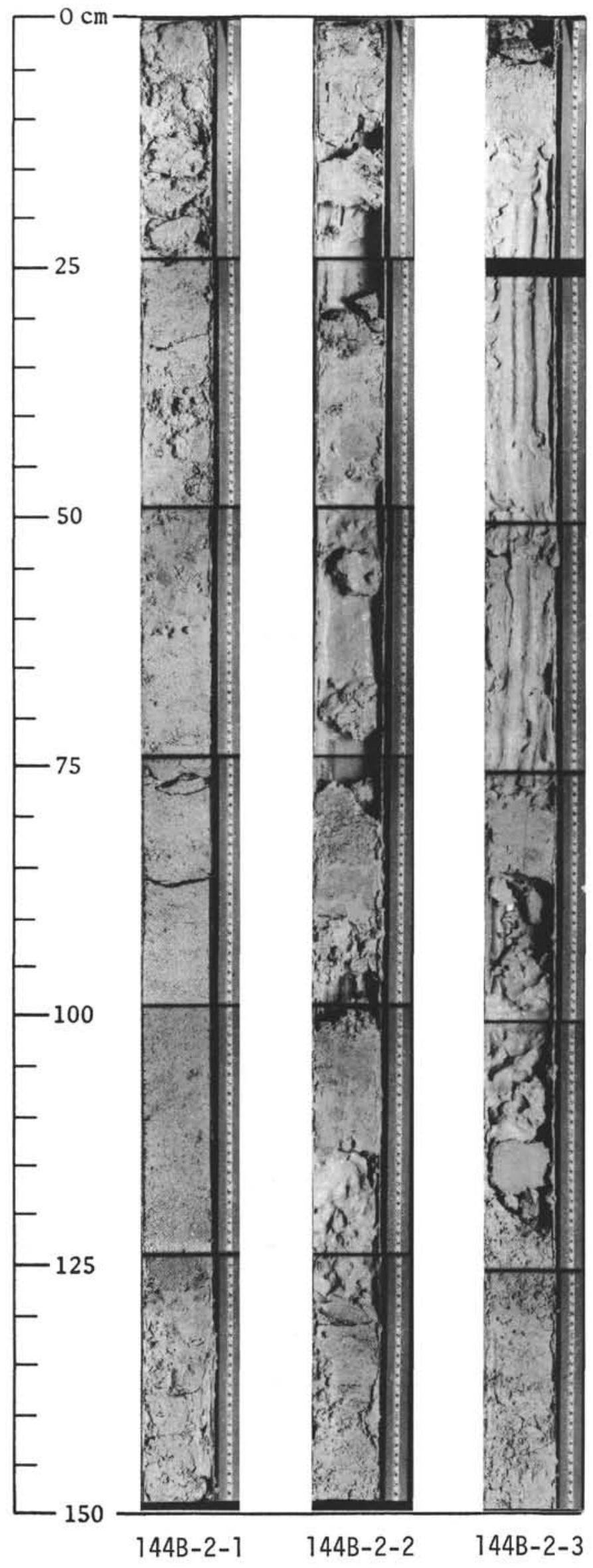


SITE 144B

CORE 3

DEPTH (m) 27-36

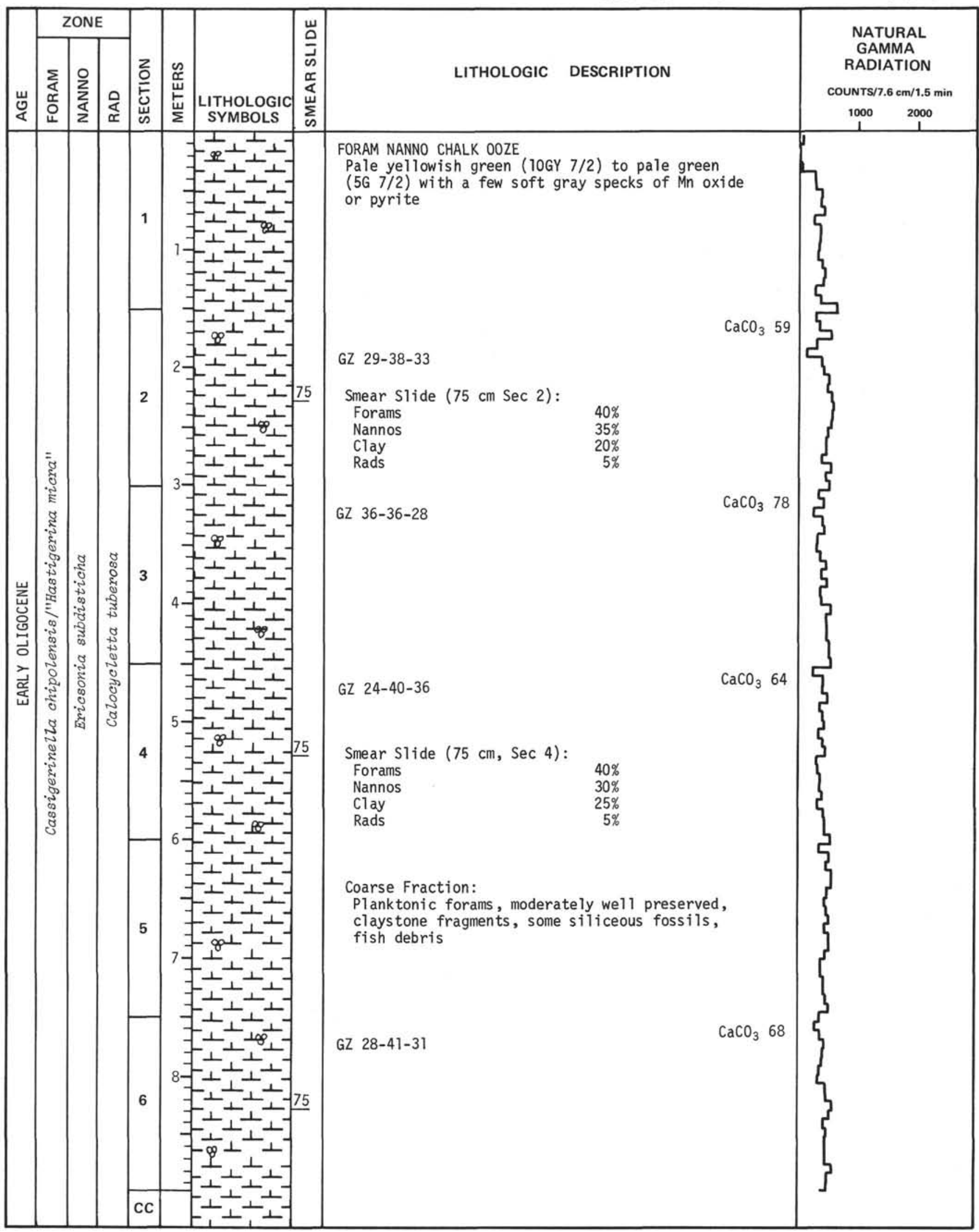


SITES 143 AND 144

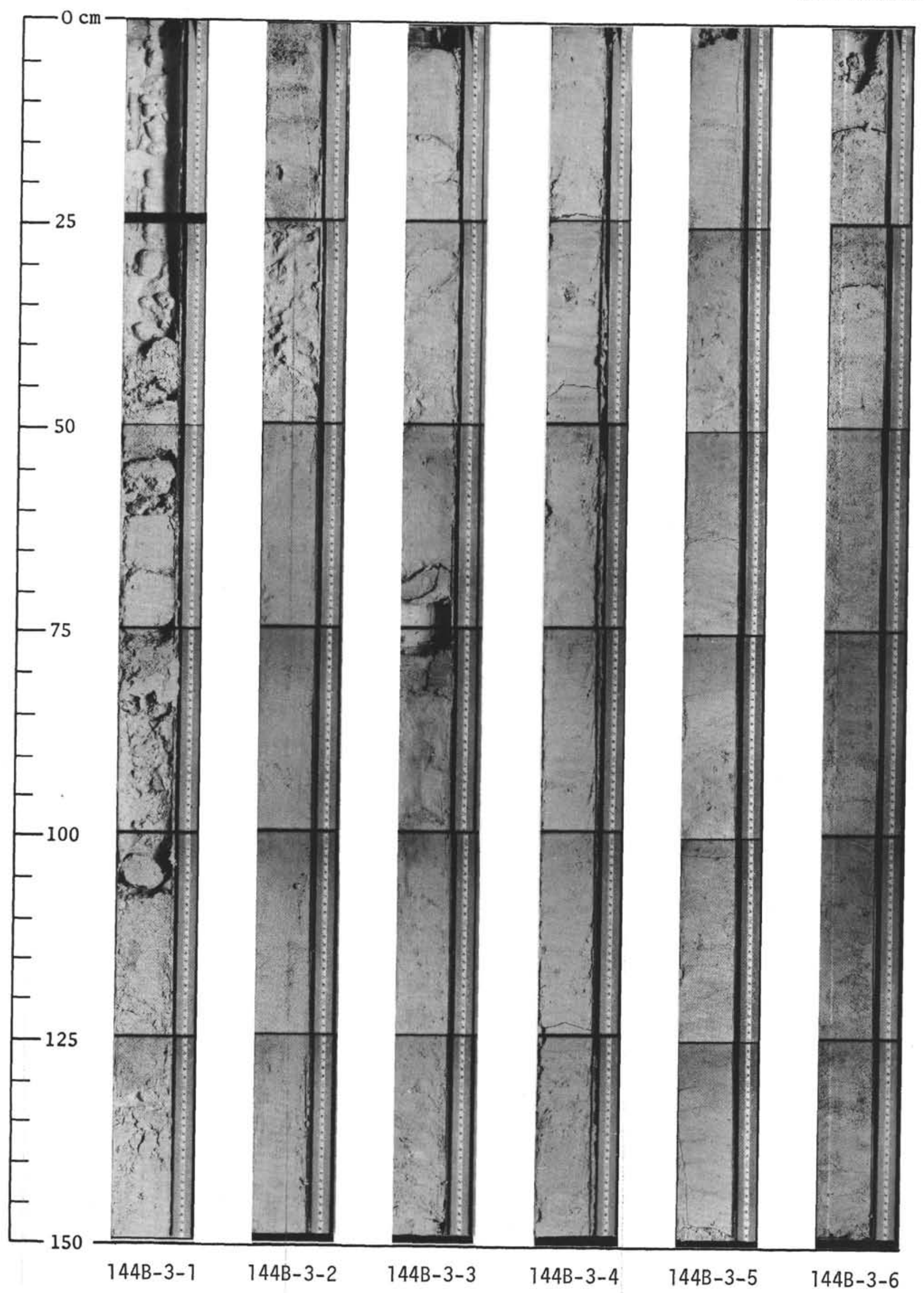




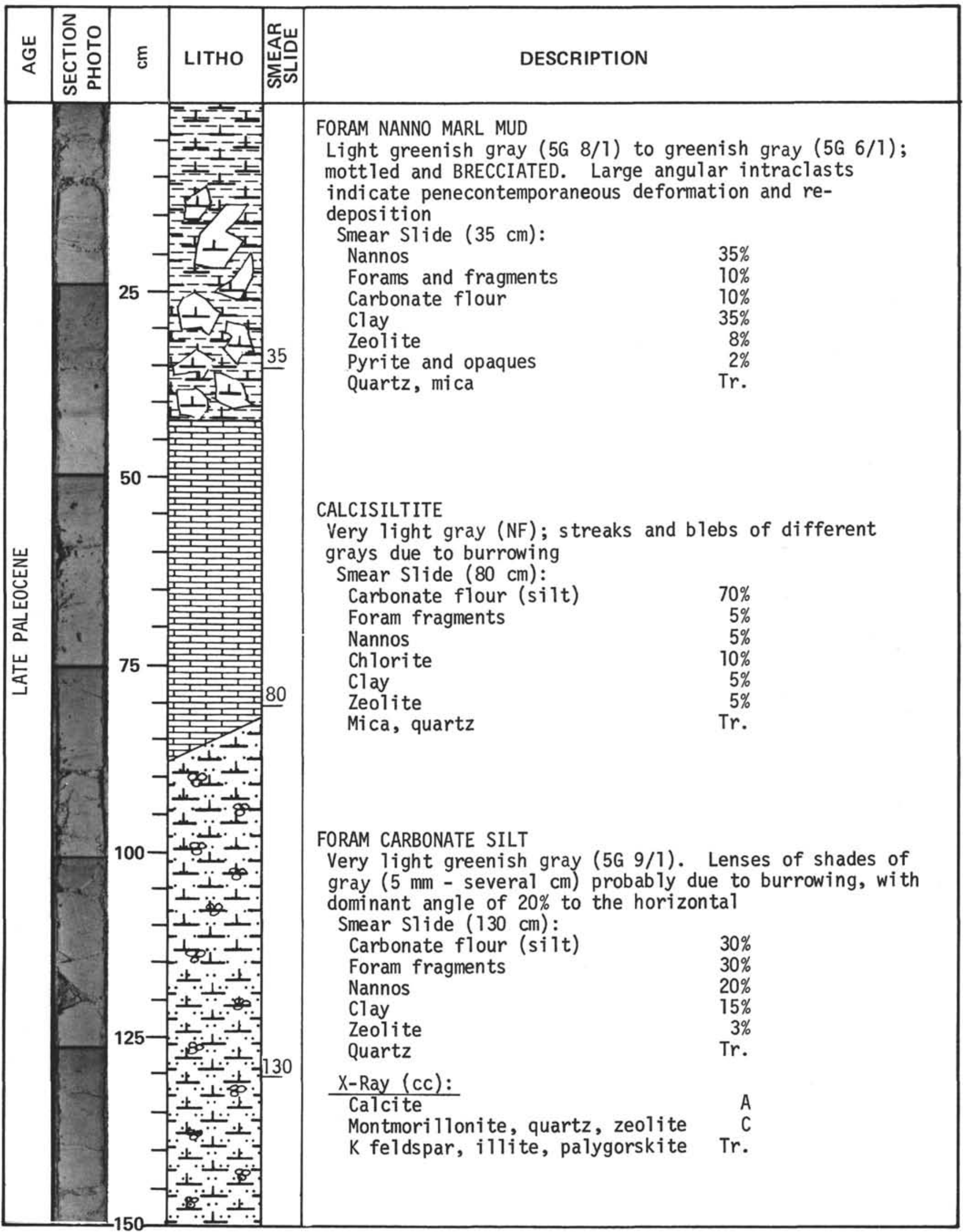

